\title{
REPORT \\ OF THE FIFTH ANNUAL ROUND TABLE MEETING ON LINGUISTICS \\ AND LANGUAGE TEACHING
}

\author{
EDITED BY \\ HUGO J. MUELLER
}

GEORGETOWN UNIVERSITY PRESS

Washington, D. C. 
Copyright 1954

Georgetown University Press

The Institute of Languages and Linguistics

School of Foreign Service

Georgetown UnIVersity

Price: One Dollar a copy 
9 and Linguistics, School of Foreign Service, Georgetown University, publishes monographs intended to contribute to the discipline of linguistics and the teaching of languages. Manuscripts should be addressed to

Leon E. Dostert, Acting Editor Monograph Series on Languages and Linguistics 1719 Massachusetts Avenue, N. W. Washington 6, D. C. 



\section{TABLE OF CONTENTS}

Page

Foneword $\ldots \ldots \ldots \ldots \ldots \ldots \ldots \ldots \ldots \ldots \ldots \ldots \ldots \ldots \ldots \ldots \ldots$ vii

INTRODUCTION

Reverend Frank L. Fadner, S.J............... 1

Archibald A. Hill, Vice-Director, Institute of Languages and Linguistics........................ 6

I. BILINGUALISM AND MIXED LANGUAGES

Problems of Bilingual Description.............. 9

A Child's Learning of Two Languages.............. 19

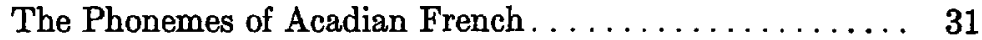

Linguistic Convergence in Immigrant America . . . . . . . 40

Discussion........................... 49

II. CULTURAL IMPLICATIONS OF LINGUISTIC

SCIENCE. . . . . . . . . . . . . . . . . . . 57

III. THE SPECTROGRAPHIC ANALYSIS OF SPEECH

Acoustical Vowel Relationship................. 62

Why and How Do We Study the Sounds of Speech?.... 73

Discussion........................... 80

Time Measurements in Speech Analysis. . . . . . . . 83

Discussion . . . . . . . . . . . . . . . . . . . . . 95

IV. LANGUAGE. AND CULTURE

Introductory Remarks. . . . . . . . . . . . . . 98

Developing Cultural Understanding through Foreign Language Study . . . . . . . . . . . . . . . . . . . 99

Towards a Cultural Theory of Lexical Innovation....... 105

Literacy as a Problem in Language and Culture........ 117

Non-segmental Elements in Foreign Language Learning. . 130

Discussion........................ 136

V. CURRENT TRENDS IN LANGUAGE AND CULTURE PROGRAMS IN WESTERN EUROPE.......... 141

VI. A HUMANIST LOOKS AT LINGUISTICS. . . . . . . . 146

Appendix 1. Program of the Fifth Annual Round Table Meeting 153 Appendix 2. Membership of the Fifth Annual Round Table Meeting. . . . . . . . . . . . . . . 155

Appendix 3. Index of Speakers. ................ 159 



\section{FOREWORD}

Since the publication of the papers and discussions of the Fourth Round Table Meeting as No. 4 of this Monograph Series, another year has gone by, and another Round Table Meeting, the fifth, has been successfully concluded. The present Monograph presents the results of this fifth meeting, held in the spring of 1954 .

This annual event has now become a well established tradition. A steady interest in the meetings among linguists and language teachers is evident in the ever-increasing number of participants and of institutions represented.

The publication of the papers, as well as of the highlights of the discussion, is well justified as a means of preserving what otherwise tends to be lost in the fleeting moments of oral presentation.

Thanks is due to those who have consistently sponsored the meetings and to whose who, by their active participation, have contributed to their fruitfulness.

Hugo Muglier, Editor. 



\section{Introduction}

The Reverend Frank L. Fadner, S. J., Executive Assistant to the Regent, School of Foreign Service, Georgetown University, made the following welcoming remarks:

It has become my privilege, by reason of the necessary absence of the President of the University, the Reverend Edward B. Bunn, sincerely and cordially to welcome you to the Fifth Annual Round Table Meeting on Linguistics and Language Teaching here at Georgetown.

The rather spectacular accomplishments of your field of endeavorthe actual possession of language skill and a scientific knowledge of the mysteries of language - have always been a source of intrigue for the laity, but I think that since the beginning of the Second World War greater store has been set by multi-language control than at any other time in our history.

Yet the mere acquisition of language skill is no guarantee that the expert will be the true man of the world we stand so much in need of today.

The attainment of proficiency in a number of languages is quite within the range of human capacity; Noadiah Moore Hill taught himself 50 languages before he died in 1889 , yet he never once set foot outside his farm in North Chatham, New York. A much less spectacular accomplishment-the control of two or three languagescauses little wonderment or awe anywhere outside English-speaking America, where, by reason of geographical and historical circumstances languages have not come to constitute a national forte.

Language itself is a tool-a means for the communication of ideas, primarily, of course, by means of sound symbols, and eventually by means of graphic signs.

So, too, the practical mastery of one or more modes of speech in addition to the one to which a man has been reared is a means to an end-the end being, in broadest terms, human understanding-a grasp of the facts of human life in the world of living men. And like every instrument, linguistic skill is an effective tool, humanly speaking, according to the wit and mentality of the speaker. 
Language is man's most intimate possession; its existence is called for by the essentially communicative nature of man, the social animal.

We ought not to forget that the man who has set out on the adventure of language in his early youth is not likely to fall heir to the sentimental provincialism of romantic nationalism and racism. His will not be the sin of the German nationalist, E. M. Arndt, who over a century ago cursed humanism and the very idea of humanity. He will not come to dissipate his emotions in the irrationally romantic hymns of a Klopstock to native land and language.

A democratic tradition demands that young people-besides those destined for the limited ranks of the highly specialized, have a knowledge of the languages and customs of the people we deal with as a nation-enemies as well as friends-Fas est et $a b$ hoste doceri. The scope of the humanistic ideal has become a very concrete thing in our day.

But if in America our education has to be geared to the demands of independence, which, I take it, means that the individual is to remain the point of stress, then especial care must be taken that the aims of language study be channelized toward the complete formation of the individual who is made of rational soul as well as body. As Bayard Quincy Morgan of Stanford University has pointed out, foreign language study and the acquisition of foreign language skill must be for education as well as training, for enrichment of the soul as well as for utility, for culture as well as mere activity. That is why, it seems to me, nothing less than complete language formation must be the goal of language study in this country-formidable though the resultant program may be.

Our educational system must be so arranged as to afford interested students the opportunity of attaining maximum performance in language; that is, in at least one other language besides the native tongue. Although this certainly means the real acquisition of the spoken language, we must not allow our American proneness to awe in the face of mechanical achievement to force us to discard the reverence we ought to have for the written record of the past. Complete control of the foreign language is the objective. The man of liberal education cannot justify that qualification by mere oral fluendy in a foreign tongue. Americans in Europe are always astounded at an ordinary tradesman's fluent command of several languages, in places 
at least, where such skill is practical. The porter who rolled my luggage up to the French-Spanish border at Hendaye spoke with equal ease in Spanish, French and Basque, although he was not able to read the written treasures of those languages. He could not be said to have a liberal education, to have a complete appreciation for the world and humanity for all his polyglot accomplishment. Someone once pointed out to Bismarck a man who knew seven languages. "Really?" said the Iron Chancellor, "What a wonderful headwaiter he would make!"

Certainly a reading knowledge, which obviously is not synonymous with mere skill in translation, must be an objective in foreign language study, for it is also through acquaintance with the record of a people's past that one gets to know the present. Language, let me repeat, is a tool. Language study and attainment are ancillary: language is the handmaid of history. A unilingual historian is a monstrosity.

Knowledge of a foreign language is a key to the vast storehouse of what a people has written about itself; the young American, in his own language, already has access to what has been penned by his own compatriots about other peoples. To come anywhere near meriting the tag "liberally educated" that young American must have facility in handling the historical, recorded products of foreign thought. And he ought to be carefully directed in the choice of language or languages he is thus to make his own. He must not forget that he belongs to a civilization which springs from Europe; to make a solid foundation for himself he should make the great Indo-European language group his laboratory. Let him choose his second language from among those spoken in an almost unbroken territory extending from western Europe to Northeastern India. Let him learn the area of the language which he has chosen-its culture, its historical past and its present. Let him use his command of the reading knowledge he has gained to deepen that acquaintance during the course of his lifetime.

If he has chosen, for example, the Spanish-speaking world, let him learn to appreciate the development of the crusading outlook of that positive race of people, the individuality of its single members, the strong moral note in a thoroughly Christian culture where Calderón recommends the doing of good even in dreams and turns the tale of Faust from tragedy into triumph. If the Russian-speaking world be- 
comes the focus of his attention, let him come to know first hand the tragic tale of people gone astray, the triumph of Sanin and Makari over Sorski and Sylvester. Let him know the artistic and the ascetic good with the political bad. Let him be the better American for it all, the keener in his esteem for the rational heritage of the West in the face of sentimental intuition's failure in the East.

Important though it is, indeed, essential to his complete formation, the American student must not rest content with mere reading knowledge as the objective attained in foreign language study. The American student must be vitally in the present. He must learn to speak and write in the languages he has determined to make his secondary vehicles of positive thought. I propose that from the very outset the student be dominated by this aim of practical conquest. The knowledge of the spoken language is proved by experience to be the most difficult skill connected with language study. The discipline involved is the most trying; it demands positive, original response on the part of the student. For that reason the aim of the earnest beginner in language must be the oral conquest of the medium. To come anywhere near the bull's eye the marksman must aim above the target.

One cannot rest content or complete with the control of the past exclusively. It is too often assumed that the masterpieces of a great literature acquaint the reader intimately with the soul of the people of the author's nation. They do, but only to the trained analyzer; a literary masterpiece, like a painting, involves the selection and heightening of detail. It is the author who talks through his characters; but the intelligent American citizen wants to talk with the people. How much more one learns about the mores and inner fibre of a nation when he hears the little Spanish boy in all simplicity explain that the Moors can't stand the sight of a swallow because that's the bird which plucked the thorns from the bleeding head of Christ; when he hears the robust wife of a bullring caretaker describe the entrance of a noble bull into the arena, or criticize the motives of a neighbor who walks through the village con intencion de Miura! What history is revealed when a sober peasant answers your enquiry after the state of his health with the remark that he is feeling very Catholic today, thanks be to God, or when his wife describes the ugliest girl in the community as a cara de hereje! Is the cold printed page of a literary masterpiece likely to give you, the reader, the same 
practical insight into the character of a race as the remark you hear and understand when a citizen of Triana describes for you the drawbridge over the Guadalquivir, which he calls Dominus Vobiscum!

The true spirit of a nation is so vividly protrayed in that almost exclusively oral creation - the country's folklore. The scientist's collection of gems in printed form are not going to bring the thrill those things offer in their vocal dress and setting. To make your own the spiritual import and the austerity of Spanish thought you have to hear and understand the Asturian swain singing to his sweetheart, with all the passion of Spanish love, swearing he'll give her his heart and his life, but not his soul, for that's not his to give, that he loves her more than life and heart $y$ más que el alma no digo, porque se lo debo a Dios: "But I don't say more than my soul, because that I owe to God."

It is sometimes objected that a stress on oral fluency in language study (and, mind you, I am not advoeating that that be our sole objective), by reason of the disciplinary demands entailed, means an expensive investment in time which will necessarily' be lost when contacts and the opportunity to chat with natives is lost. I say that the man who has lived the terrific human experience of talking and understanding and writing in a tongue to which he was not born will not willingly let that triumph die. He will continue the enriching contacts he has made with a people; he will not let his active citizenship lapse. He will maintain his membership even if it means no more than periodic visits to Little Italy or China Town in his own American community. It is true that the drilling and discipline he's had to submit to while he was learning to talk may not have given him the leisure to read the nation's masterpieces, but he'll always be able one day to indulge himself in that slippered ease. The serious student will never give up the hardwon talent; he'll live on listening with his ears to living representatives of a culture and will inevitably want to browse about, too, in the treasure heap left behind by its great formal artists of the past.

The educated, humanized American must be a positive, active, productive citizen of two countries-the realm of the past and that of the present. If he stays only within the limits of his own language empire-geographically vast though it may be-he cannot hope to see the whole picture of even that single empire's past or present. 
The principle of contrast demands a foil upon which to define and heighten the essentials in the story, past and present, of his own neighborhood. The intelligent and deepened love borne by the returning pilgrim for his native land is a commonplace of human experience. And the more worlds that fall to the student's spiritual conquest (and life is too short for their number to be great), the more costly victories won, the brighter and clearer will be his own picture. But the victories must be complete. The young conqueror must remember that exclusive stress on speaking without attention to the written word's control may make him but a tinkling cymbal, speaking the tongues of men without the spirit of charity demanded in the effort to understand facts-the love and will to understand yet another human tongue-the impulse which spelled the glorious defeat that crowned the efforts of a Noël Chabanel among the Indians of New York.

Ladies and Gentlemen:

It is the earnest wish of your hosts-the Administration and Faculty of the Institute of Languages and Linguistics of Georgetown University's School of Foreign Service, that you may find real profit in these two days of discussion at our annual round table meeting.

\section{Dr. Archibald A. Hill, Vice-Director of the Institute of Languages and Linguistics:}

Our task this morning is first, to express regret for a number of absences. We can certainly express that regret very deeply without at the same time prejudicing our feeling of gratitude for how well some of the absences have been filled. The absence of Father Bunn and of Father Walsh are those to which I refer. There is no one who could have spoken so well for both as Father Fadner who has just addressed us. The third absence, which needless to say I regret even more personally, since I am acting as the inadequate substitute, is that of our Director, Professor Dostert, who has been more than any other, the mind and heart of these conferences.

I will not hold you long, but I must at least say a little about the rationale of these conferences and of this Institute. I think one could state it very briefly in a sentence. The conferences are devoted to the simple proposition which ought to be self-evident, but often seems to be forgotten-namely, that linguists, who have the reputation of being very difficult people indeed, are after all human beings, and that in 
the proper atmosphere we can even get along with our much more practical and valuable colleagues, the teachers of languages. It is for that reason we gather here together this morning a rather special kind of audience, consisting, first, of people whose interest is in the propagation of the kind of knowledge of which Father Fadner speaks, the ability to use foreign languages, not merely to decipher them, but to use them, and second, persons whose interest is in the investigation of the nature and structure of such systems of communication as go by the name language. We believe that gathering together people of these two groups, always with the paramount interest of increase of knowledge and of human skill in this sphere, cannot but bring good to both parties.

We might say of the Institute that we try to do something here which is not unique in any of its parts, but which we think of, or like to think of, as nearly unique in their combination. First of all, we bring together people who have a perfect, because a native, command of the languages taught. These are the models which the students follow. We bring together also people who have had many years of the practical skill of teaching foreign languages in the American academic world. And the third sort of person is the trained linguistic analyst whose job it is to try to understand what the structure, what the framework and what the separate parts of a given language are. We believe that by gathering these people together, each making his own contribution, each respecting the other, the end result of our teaching process is somewhat better than it would be if any one of the three were left out. We believe, further, that though no machine is ever a substitute for a human being, machines and audio-visual aids can be very healthy additions to the human teaching and analytical skills upon which we basically rely. And finally, we believe that it is necessary to examine and re-examine constantly our methods of teaching, what we teach and what we teach for, to see that eventually our knowledge of language fits indeed, as Father Fadner has suggested, into a framework of broad human understanding and communication. Languages cannot be taught in a vacuum, and a language skill acquired merely as an ability to order a meal in Paris or Bankok is about as valuable as what might be done merely by sitting down in your private room with a dictionary and looking up the corresponding words. If, however, our language knowledge leads into an understand- 
ing of the whole structure of values, the whole structure of ways of doing and of thinking things in other countries, with an increased respect for the basic belief that men are everywhere different and everywhere the same, then perhaps we have contributed something to the ideal which we all serve-liberal education. It is in this spirit then, that we welcome you to this our Fifth Conference, and thank you for the contributions which you in your discussions and in your lectures will make to the problem which we continually find ourselves working at. It is my very great pleasure then, to welcome you all to this our Fifth Round Table Conference. 


\title{
I. Bilingualism and Mixed Languages
}

\section{Problems of Bilingual Description}

\author{
EINAR HAUGEN \\ University of Wisconsin
}

The theme of our conference this morning is one with many facets. We are here going to give special emphasis to the aspect of bilingual description. The problems of descriptive technique have been much discussed in recent years, but few have considered the problems involved when we try to describe more than one language or dialect at a time. We need to extend the concept of description to include the issues which arise when two or more languages are used by the same speakers. We need to study the methods that are appropriate for making systematic comparisons of languages and dialects without regard to their genetic relationships. For such comparisons I shall here use the term bilingual description. The study of linguistic borrowing is a part of historical linguistics; but at the moment of borrowing we have a linguistic state which can be studied for its own sake, namely the co-existence of different linguistic structures in the same speakers. The interferences which result in borrowing are evidence of inter-linguistic identifications made by these speakers. The goal of bilingual description should be to predict (and in some cases prevent) these interferences by describing the identifications that may be expected. A bilingual description is thus more than two monolingual descriptions laid side by side, for it attempts to equate units of the one language with units of the other. In so doing it can be strictly synchronic in its procedure and should be applicable to any two languages or dialects. Foreign-language grammars and bilingual dictionaries are more or less explicitly based on bilingual description and should profit from an exploration of its principles. The historical results of bilingualism which turn up in every language as loanwords and loanshifts present us with material for testing our hypotheses. But we need not wait for history; we can test them ourselves by trying to teach the languages to monolingual speakers.

We may begin by disposing of a theoretical difficulty which is 
raised by the current conception of the phoneme as a unit limited to a given linguistic structure. If the units of a structure are derived exclusively by the comparison of utterances in a given dialect or idiolect, how can we compare it to the units of structures derived from the comparison of entirely different utterances? Before the rise of structural linguistics the problem was less complicated. The phonology of a language was assumed to consist of sounds; the sounds of one language were interpreted in terms of the sounds of another, and that was that. Linguists were only a shade more sophisticated than the native speakers and were themselves subject to some of the same interference effects. Today most phonological descriptions are organized around the phoneme concept, and it will seem natural for most linguists to compare the phonemes of one language directly with those of the other. But, as Weinreich points out in his recent book Languages in Contact, the phonemes of one language are by definition incommensurable with those of all others. He solves the problem by accepting a dichotomy of form and substance, assigning the phonemic structure to the former, and the interlingual identifications to the latter. "The actual sounds produced by the bilingual," he writes, "lie, as it were, in the structural no man's land between two phonemic systems." In another passage he writes that it is their "physical resemblance" which "tempts the bilingual to identify the two phonemes astride the limits of the languages." Whether or not we accept this formulation, it points up the importance of allophonic and distributional data in the making of a bilingual description. If the identifications are due to purely physical resemblance, our description must take full account of the physical nature of the phonemes. This is no calamity, particularly if it be true, as Harris writes in his Methods of Structural Linguistics that "the ultimate elements of the phonology of a language, upon which all linguists analyzing that language could be expected to agree, [are] the distinct (contrasting) segments (positional variants, or allophones) rather than the phonemes."3

The problem may be illustrated by referring to an article exploring the bilingual description of English versus Spanish, Chinese, and

1 Languages in Contact (New York, 1953), 14.

Ibid. p. 7.

3ethods in Structural Linguistics (Chicago, 1951), 72 fn. 28. 
Portuguese by David W. Reed (assisted by Robert Lado and Yao Shen). ${ }^{4}$ The phonemes of these languages are here presented in parallel phonetic charts, followed by a discussion of the special difficulties of Spanish, Chinese, and Portuguese speakers in learning English. I am struck by the lack of predictive correlation between the charts and the difficulties. Some are of course obvious, but there are puzzling cases. English and Chinese are both said to have the phonemes /l/ and $/ r /$, yet Chinese speakers are said to confuse the English sounds, so that pull and poor may sound alike. A similar description of German and English would have to state that both have the phonemes /s/ and $/ z /$; yet we all know that the confusion of these is a common weakness in the German pronunciation of English. The key to the problem is of course the need for a complete statement of phonetic qualities and distributions. It is not enough to know that Chinese has an l-phoneme; we also need to know where it occurs, and whether it is likely to be physically identified with English / $1 /$ or $/ r /$. These are the realities which the learner has to face, and it leads one to question whether a phonemic transcription is likely to be the most useful kind of transcription in a foreign-language text. This very question is raised by Yao Shen in a recent article, and she concludes that departures from phonemic transcription are urgently called for when it is likely to lead to the learner's confusion. ${ }^{5}$

Once we have gathered our basic phonetic and distributional data for each of our languages, we are prepared to make comparisons of allophones position by position. But we will of course not wish to leave our data in the relatively complex form of an allophonic comparison. We wish to set up unit formulas of the greatest possible generality. If, as often happens, all the allophones of one phoneme in one language may be expected to be identified with the allophones of a single phoneme in the other language, they show a pragmatic equivalence which deserves a name. I shall here call this a diaphonic relationship and describe the two phonemes involved as diaphones of one another. The term "diaphone" was used some years ago by Daniel

- The Importance of the Native Language in Foreign Language Learning, Language Learning 1.17-23 (1948); cf. also Yao Shen, Initial / $r$ / in American English and Mandarin Chinese and How to Teach it, Ibid., 2.47-55 (1949).

- Departures from Phonemic Representations, Language Learning 4.83-91 (1952-53). 
Jones to refer to the sameness of phonemes in different dialects and can well be extended to refer to different languages as well. Such a diaphonic relationship exists, e.g. between English /b/ and Norwegian $/ \mathrm{b} /$, and can be expressed in a formula of the following type: $\mathrm{E} / \mathrm{b}>\mathrm{b} / \mathrm{N}$. This means that English $/ \mathrm{b} /$ is interpreted by Norwegian speakers as the counterpart of their own $/ \mathrm{b} /$; the arrow has no historical significance, but means "is identified with" and points every- where from the secondary language to the primary one. It can be reversed when one is referring to the interpretation of Norwegian /b/ by English speakers: ${ }_{N} / b>b / E$. Such a one-to-one reversible relationship is not the only, or perhaps even the most common one. Very often two or more phonemes in one language are identified with one

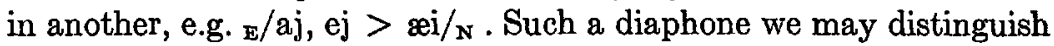
by calling the former a simple diaphone, the latter a compound one. Since the two English phonemes here converge to one Norwegian phoneme, we may call this a convergent diaphone, in contrast to a divergent one like the formula $\mathrm{E} / \mathrm{u}>\mathrm{u}, \mathrm{o} / \mathrm{N}$. Whenever the allophones of a phoneme become members of different phonemes in the new language, we may set up a complex diaphone of the following type: ${ }_{\mathrm{I}} / \mathrm{b}_{\mathrm{v} 1}>\theta ; \mathrm{b}_{\mathrm{vd}}>\delta / \mathrm{s}$, which merely says that Icelandic $[b]$ and $[\delta]$ are one phoneme, but that they will be interpreted as two by English speakers of Icelandic. On occasion we may even get a complex diaphone with compound members, e.g. $\mathrm{s} / \hat{s}>\emptyset, 0$, a, a; $\partial>\mathrm{e}, \mathrm{a} / \mathrm{s}$. The commas in general mean free variation, the semicolons allophonic variation. Compound and complex diaphones may also be reversible, but are not necessarily so. While this is not the place to discuss it, a similar terminology could be worked out for morphemes, using the term diamorph for morphemes which are identified across linguistic borders.

We may now raise the second of the main questions in bilingual description: how likely are different investigators to arrive at the same diaphones? Much will here depend on their choice of phonemic principles for monolingual description. In order to illustrate the problem involved I shall present a small, but detailed example of bilingual description drawn from materials that I have myself collected. We shall try to describe the diaphones of quantity in the vowel nuclei of English and Norwegian, as these might be predicted by linguistic analysis, and check this with what has actually been found in the 
speech of immigrants. Much of the material has been published in my recent work, The Norwegian Language in America, but will here be taken up for renewed interpretation. ${ }^{6}$

Since Norwegian is the primary language in this comparison, I shall describe the situation in that language first. The Norwegian speakers here described approached the learning of English with a vowel system in which there are 9 simple vowels / a e i o $\mathrm{u}$ y $\varnothing \varnothing$ a/ which can occur either long or short and 3 native diphthongs /æi $\varnothing y \emptyset \mathrm{u} /$ which occur only long. Length is traditionally regarded as a phoneme which may be written as a colon/:/ after the simple vowels. It occurs only in connection with stress, however, and is then in complementary distribution with consonant length, so that there are two kinds of stressed syllables, which may be written $/ \mathrm{V}:(\mathrm{C}) /$ as in /ba:ken/ 'the behind' and /VC:/ as in /bak:en/ 'the hill'. This means that all non-Norwegian vowel nuclei must be reproduced with a long or a short vowel, and in the latter case the consonant must be extended. Now as we have seen, there is a clear structural parallelism between long vowels and diphthongs, so that one could also consider the long vowels as geminated. This would eliminate the phoneme of length, since the long consonants can also be described as geminated, and the more readily so as they are usually written double in the standard orthography. This would add 9 vowel clusters to the previously existing 3 , and would require one to say that foreign vowel nuclei must be interpreted as either simple or complex, with a complementary interpretation of following consonants. Once we have taken this step, however, we can go still further and recognize the possibility of regarding the long nuclei as consisting of vowel plus consonant, or semivowel. In this case we would have to distinguish between the high phonemes and the rest. The four high vowels /i y u o/ fall into two groups, those with (a very slight) final tongue-tip glide and those with final labialization, so that they could be written respectively / ij yj/ and / uw ow/. The mid and low vowels have, if anything, a low or centering offglide, and would therefore in this interpretation be written /eh $\varnothing \mathrm{h}$ ah $æ \mathrm{~h}$ ah/. The diphthongs would be written /æj $\varnothing \mathrm{j} \emptyset \mathrm{w} /$. There are arguments against adopting this interpretation for Norwegian, one being the absence of prevocalic $/ w /$, another the complication of the consonant clusters, a third the loss

- Philadelphia, 1953, pp. 419-32. 
of parallelism between length in vowels and consonants. But I do not wish to argue the merits of one phonemic analysis over another. I merely wish to show how each of these interpretations changes the picture of the bilingual description.

It will be no secret to those present that English vowel nuclei can also be described in various ways. ${ }^{7}$ In fact, it was the wide disagreement in recent phonemic descriptions that led me to prefer the traditional IPA symbolization in my book. None of the new descriptions seemed to fit my dialect precisely, and the Kenyon version of the IPA at least provided me with symbols which clearly distinguished all the nuclei from each other. For my dialect, which may be described as a variety of general midwestern, it provided 11 vowels [I $\varepsilon$ U $\Lambda$ a ? $i$ e $o u$ ] and 3 diphthongs [ar av or], all listed in the first column of the accompanying chart. ${ }^{8}$ Two major alternatives to this transcription have been provided by other investigators. One is that offered by Swadesh, essentially one in which the first four listed above are regarded as simple, while the rest are regarded as complex and written as clusters of two vowels each. Since my dialect is basically similar to Swadesh's, this would be a satisfactory method of writing my dialect also. But then the 14 nuclei listed above would consist of only 4 vowels / i e u $ə$ / and would have 10 diphthongs /ææ aa oo ii ei ou uu ai au oi/.9

Another, and more generally accepted reinterpretation of the vowel nuclei of English, is that initiated by Bloomfield and developed by Bloch, Trager, and Smith. In some of its early forms this was quite unacceptable as a representation of the dialect here described, but now that it has ceased to be a phonemic analysis of one dialect and has turned into a transcriptional arsenal on which any speaker of

${ }^{7}$ Leonard Bloomfield, Language (New York, 1933), 91; Bloch and Trager, Outline of Linguistic Analysis (Baltimore, Md., 1942); Haugen and Twaddell, Facts and Phonemics, Language 18.228-37 (1942); Martin Joos, Studies in Linguistics 2.44 (1943); Morris Swadesh, On the Analysis of English Syllabics, Language 23.137-50 (1947); Kenneth L. Pike, On the Phonemic Status of English Diphthongs, Language 23.151-9 (1947); Trager and Smith, An Outline of English Structure (Norman, Okla. 1951).

${ }^{8}$ I was born and brought up in Sioux City, Iowa, educated in Iowa, Minnesota, and Illinois; have taught in Wisconsin since 1931.

- The symbols $æ$ and $o$ have here been substituted for Swadesh's $\varepsilon$ and 0 , and ei ou for ee and oo. 
English can draw, it is at least one acceptable mode of presenting the data of the dialect. In this system there would also be four short nuclei, written /i e $u$ ə/. But the long nuclei would here consist of vowel plus consonant, the consonants in each case being one of the semivowels $/ \mathrm{h} \mathrm{j} \mathrm{w} / .^{10}$ The accompanying chart shows the relationship between this transcription and the others. The peculiarity of the semivowel $/ \mathrm{h} /$ is the fact that its phonemic status is in doubt. It can be said to occur with the short nuclei, but only before $/ r /$, in words like beer, care, poor, turn. On the other hand, the vowels $/ \mathfrak{x}$ a o/ do not occur without it. There is no distinction of length between bomb and balm, can and can, pot and bought. In this transcription the long vowels [i e o u] are split into vowel plus glide /ij ej ow uw/, and are therefore classed with the obvious diphthongs /aj aw oj/.

Even from this sketchy outline of the vowel nuclei of the two languages we could probably make some shrewd guesses concerning the bilingual treatment of quantity by Norwegian speakers. The English nuclei fall into four classes, which are separated by bars in the accompanying chart: (1) those that all agree in describing as short, or unitary, viz. /i e $\mathrm{u} \partial /$; (2) those that have allophonic length, viz. $/$ × a $\% /$, plus the four preceding ones before $/ \mathbf{r} /$; (3) those that are held to be units by some, geminated vowels or vowel plus semivowel by others, viz. /ij ej uw ow/; (4) those that all agree in describing as diphthongal, viz. /aj aw oj/. Since the vowels of class 1 are short and occur only before consonants, we might expect them to be identified by Norwegians with their own short vowels, of which the same is true. This is correct, but in such cases the Norwegian structure requires an extension of the following consonant: picnic, bet, bull, husk $>$ /pikknik, betta, bull, hasska/. We might further expect that the elements which some have described as length in the American vowels would be identified with length in Norwegian, since the latter can also be regarded as an extension of the short nuclei and occurs before juncture. Since Norwegian rarely has length before consonant clusters, we might expect that the lengthening element would disappear in this position. This, too, corresponds to the observed facts of interference, except for one point, as we shall see.

The vowels of class 2 are identified as long in words like add,

${ }^{10}$ The use of $/ \mathrm{j} /$ rather than $/ \mathrm{y} /$ is due to considerations affecting the symbolization of Norwegian. 
THE DIAPHONES OF ENGLISH > NORWEGIAN

Stressed Vowel Nuclei

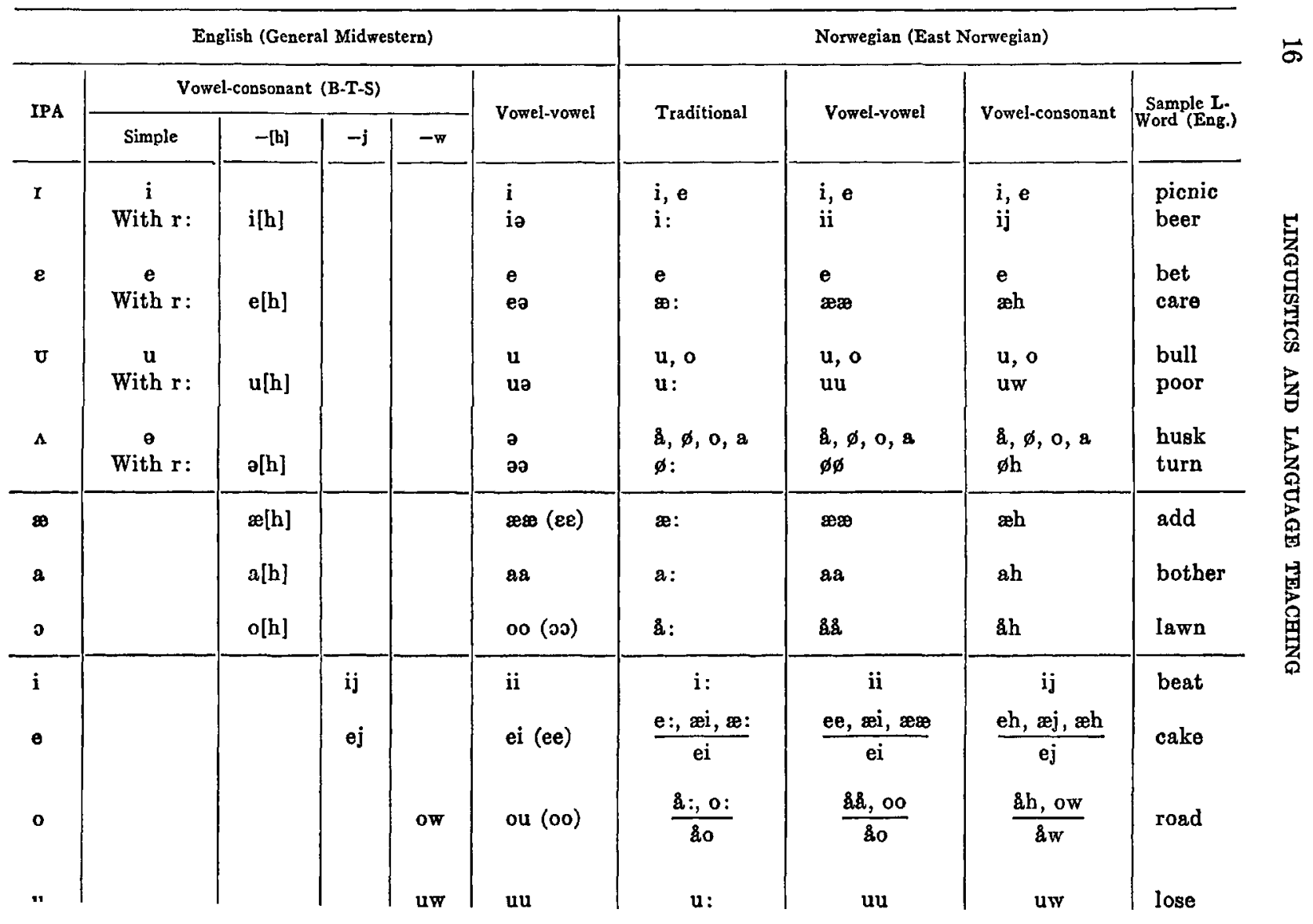




\begin{tabular}{|c|c|c|c|c|c|c|c|}
\hline $\mathrm{ai}$ & aj & & $a i$ & $\frac{\mathfrak{x i}}{\mathrm{ai}}$ & $\frac{\mathfrak{x i}}{\mathrm{ai}}$ & $\frac{\mathfrak{x j}}{\mathbf{a j}}^{\text {. }}$ & ripe \\
\hline$\alpha \delta$ & & aw & $\mathbf{a u}$ & $\frac{\varnothing u}{a 0}$ & $\frac{\phi u}{a 0}$ & $\frac{\phi w}{a w}$ & flour \\
\hline OI & oj & & $o \mathrm{i}$ & $\frac{\phi y}{8 \mathrm{i}}$ & $\frac{\emptyset \mathrm{y}}{\mathbf{a} \mathrm{i}}$ & $\frac{\phi j}{a \mathrm{j} j}$ & joist \\
\hline
\end{tabular}

Vowel STRUCTURes

(1) Transcribed as Vowel-vowel

\begin{tabular}{lcc} 
ai & oi & \multicolumn{2}{c}{ au } \\
i(i) & & u(u) \\
e(i) & ə & $o(\mathrm{u})$ \\
æ[æ] & a[a] & $o(\mathrm{o})$
\end{tabular}

(2) Transcribed as Vowel-consonant

$\begin{array}{ccc}\text { aj } & \text { oj } & a^{a w} \\ \mathrm{i}(\mathrm{j}) & & \mathrm{u}(\mathrm{w}) \\ \mathrm{e}(\mathrm{j}) & \partial & \mathrm{o}(\mathrm{w})\end{array}$

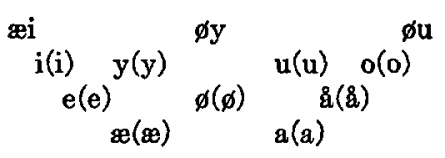

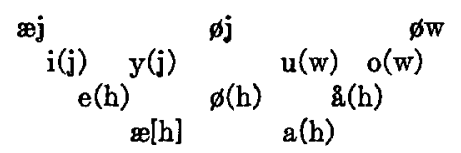

Note on symbols: [ ] enclose allophonic length; ( ) enclose phonemic length; Norwegian symbols below the bar refer to the clusters imported from English. 
bother, lawn > /æ:da, ba:der, lå: $\mathrm{n} /$, and before $/ \mathrm{r} /$, as in bar, chores $>/$ ba:r, çå:ș)/. Before clusters which remain clusters in Norwegian they are identified as short, in words like candy, bo $\dot{x}$, cord $>/$ kenndi, bakks, kårrd/. But they are also frequently identified with short vowels before single consonants, e.g. in words like black, map, cob $>$ /blekk, mapp, kabb/. While this occurs chiefly before voiceless consonants, the word $c o b$ shows that it is not a universal rule. If we turn to the vowels of class 3 , we find a similar situation, but here the vowels are nearly always identified as long before single consonants. The examples in the chart become /bi:ta, ke:k, rå:d, lu:sa/, while before clusters we get examples of shortening in words like beans, rails, toast, tools $>$ /binns, rells, tosst, tulls/. Sporadic examples of shortening also occur before single consonants, as in reap, plate, grocery, stoop $>$ /rippa, plett, gråsseri, stupp/. Only one of these is ever identified as a diphthong, viz. /ej/ in words like frame, jail $>$ /fræim, jæil/. Class 4 nuclei, however, are always identified as diphthongs, so that the examples in the chart are reproduced either as Norwegian diphthongs in ripe, flour, joist > / ræip, fløur, jøyst/ or by newly created diphthongs imitated from the English ones as in friedcake, county, spoil > /fraidke:k, kaonti, spaila/. On this, as on some of the other identifications listed above, there is considerable variation.

Any attempt to symbolize the diaphonic relations here outlined will involve us in the controversies concerning the best transcription of each language. Each system distinguishes between simple and complex nuclei, which may be designated as respectively V and VV (or for the B-S-T transcription as VS). The IPA system, with its 11 simple and 3 complex nuclei will give us the following formulas: (1) ${ }_{\mathrm{E}} / \mathrm{V}(*)>\mathrm{V}:, \mathrm{VV} ; \mathrm{V}(\mathrm{CC})>\mathrm{V} ; \mathrm{V}(\mathrm{C})>\mathrm{V}, \mathrm{V}:, \mathrm{VV} / \mathrm{N} \cdot(2)_{\mathrm{E}} / \mathrm{VV}$ $>\mathrm{VV} / \mathrm{N}$. If Norwegian long vowels are transcribed VV, we can simplify the formulas as follows: (1) ${ }_{\mathrm{E}} / \mathrm{V}(\not{ })>\mathrm{VV} ; \mathrm{V}(\mathrm{CC})>\mathrm{V} ; \mathrm{V}(\mathrm{C})$ $>\mathrm{V}, \mathrm{VV} / \mathrm{N} \cdot(2)_{\mathrm{E}} / \mathrm{VV}>\mathrm{VV} / \mathrm{N}$. If Norwegian long vowels are transcribed VS, this would mean only the substitution of VS for VV in each case. If we adopt one of the newer interpretations of English, we move the complexity of the first formula into the second: (1) ${ }_{\mathrm{E}} / \mathrm{V}>\mathrm{V} / \mathrm{N} \|(2)_{\mathrm{E}} / \mathrm{VV}(*)>\mathrm{VV} ; \mathrm{VV}(\mathrm{CC})>\mathrm{V} ; \mathrm{VV}(\mathrm{C})>\mathrm{V}, \mathrm{VV} / \mathrm{N}$. If we use the VS interpretation for either or both languages, the VV would simply be exchanged for VS in each case. There is of course no 
need of pairing transcriptions; there is much that speaks for a vowelsemivowel transcription in English as against a vowel-vowel transcription in Norwegian. In no case do the formulas do justice to the full complexity of the situation, and each transcription has its advantages. The IPA transcription does not bring out the similarity of behavior between classes 2 and 3 , and the others do not bring out the difference between the classes 3 and 4 .

I shall not be surprised if some of you have found this variety of transcriptional possibilities confusing. I have neither the time nor the inclination here to argue the merits of any one system, or to explore the question of whether diaphonic relations should be permitted to affect our choice of transcription. I will only say that a bilingual textbook would be well advised to base its transcription on diaphonic considerations rather than purely phonemic ones. My purpose has merely been to show how differences in phonemic transcription can affect our statement of diaphonic relations.

In spite of this great divergence, we must not forget that there is little disagreement on the basic phonetic and distributional facts. In the final analysis, the differences in phonemic statement are relatively superficial and will probably be ironed out as more research is devoted to this problem. The main ideas I wanted to leave with you today are (1) that a synchronic approach is possible in the study of bilingual phenomena; (2) that the identifications made between different phonemic systems by bilingual speakers can be predicted by a careful bilingual description; (3) that these can be tested by experimentation and observation, and can then be stated as diaphonic formulas in which the phonemes of the respective languages constitute the terms.

\title{
A Child's Learning of Two Languages
}

\author{
W. F. LEOPOLD
}

\section{Northwestern University}

In the area of bilingual learning of small children, the problems of description are complicated by the fact that infants exposed to two languages from the beginning do not learn bilingually at first, but 
weld the double presentation into one unified speech system. A further complication in the case studied by me, the language learning of my daughter, is the fact that English and German are so closely related that the structural differences cannot be reflected in the primitive early speech of a child. I am going to separate the initial formative stage of the first two years from the later phases of learning.

The small child first learning the language of the environment faces an enormous task. Subconsciously he must make a complete structural analysis of the language and slowly, step by step, learn to imitate the system in active speaking. In the field of sounds and words, the process goes hand in hand with the gradual development of perceptive and articulatory faculties. In the higher reaches of the syntax, which cannot be learned by imitation of relatively tangible material (sounds), but requires the grasp of abstract patterns, growth of intellectual maturity is a prerequisite for successful learning.

No wonder then that in the learning of language patterns the child appreciates and reproduces at first only the coarsest contrasts. It takes time and much effort to learn ever finer subcontrasts, until eventually the whole complex structural mechanism of the developed language is assimilated. This process of learning first rough contrasts and then finer and finer ones can be demonstrated in the learning of phonemic patterns: in vowels and consonants, in syllable and word structures. It operates also in syntax. After a long stage of speaking in single words, the art of putting two, three and more words together in a sentence is slowly learned. The relationship between the words in the sentence is not always the same. Such standard patterns as subject-verb-object are gradually learned as vehicles of communication, long before any formal indication of the relation is attempted, with the exception of word order. As the last step the morphological devices which serve as the formal signs of syntactic relations are also learned, often in the third year of life. All this refers to the learning of the patterns of one language. Bilingualism, the separate learning of two sets of patterns, amounts to a further refinement of contrasts, which is naturally learned late.

During the first two years of life, on which I have concentrated my attention in my study, no clash of phonemes due to the presentation of two languages can be said to have occurred. The phonemic structures of German and English are roughly the same, and "roughly" is 
the only measure that can be applied to a small child's language learning as long as only the coarser contrasts of the model are appreciated and imitated. Of course there are certain phonemes in the use of which the two languages do not agree, like the English dental fricatives and the vowels [æ] of hat and [ $\Lambda$ ] of but or butter, and the German rounded front vowels and voiceless domal fricatives (the $i c h$ and ach sounds). But these are all sounds which are late in the learning of monolingual children. They are normally not acquired before the third year.

In examining the sound learning of my daughter during the first two years, I paid special attention to possible bilingual effects, and found very little. Practically the only feature worth noting was the treatment of the consonant [1]. It is one of the more difficult sounds; it was not learned well during this early stage. In initial and medial position it was usually replaced by the easier glide [j]. In terminal position it was represented by vowels, and here a difference between the two languages showed up. In German words the flat [1] was rendered by the front vowel [I], whereas the English [l] with its raised back tongue was rendered by the vowel [u]-most strikingly in the word Ball, which was [bar] for the first ten months of the second year, but then became [bau] because the child had switched from the German model to the English ball. Since the diphthongs [ar] and [av] were otherwise not confined to one of the two languages, no phonemic separation resulted. At the end of the second year the [1] was sometimes reproduced correctly in initial and medial position. The child then heard terminal syllabic and consonantal [1] and sometimes reproduced it as [l], but it could not yet stand in terminal position. She added the vowel substitute to the [l], bottle becoming [balu], oil [?orlo]. The German word in which medial [1] was frequently rendered as [1] was alle. The consonant was however at first the velarized English [1]; via the palatal substitute [j] it developed into the correct flat German [1] in the last two months. There was only one English word in which medial [1] was not omitted or rendered by the normal substitute [j], namely hello; its [1] was the correct English variety. Thus the child used the two kinds of [1] at first as allophones of one phoneme, without separation by languages. At the end of the second year the models began to be followed more accurately, but the dearth of examples and the subsequent history make it hazardous to speak 
of two phonemes. Normally [j] was the substitute for [1] in both languages, and the substitutes for terminal [1] tended eventually to settle into [a] in both.

Another phenomenon worth a glance is the habit which this child developed of using a glottal stop as the introduction of any German or English word beginning with a vowel. Since this is the rule in standard German, the thought arose that it might be an influence of German on her English. That may be the correct explanation. But on the other hand this child's syllable pattern was consonant-vowel for many months. Practically no words began with a vowel, and terminal consonants were not added until the latter months of the second year, and then by no means always. This syllable pattern favored the use of the introductory glottal stop, which is of course quite common in English in isolated words emphatically pronounced. When the child's speaking became rather suddenly more fluent, at the age of 2 years and 4 months, and the syllable patterns became more varied, the use of the glottal stop receded markedly. Again we cannot speak with assurance of bilingual interference in the child's phonemic system.

A further rule of the child's speech as late as the third year was that consonants at the end of words, after they began to be added to the syllable pattern, could only be voiceless; voiced consonants of the English model were either omitted or lost their voice. This is again a rule of German, not of English. But I find terminal devoicing in the literature of child language for monolingual children speaking English and French. It is a special category of assimilation which cannot be attributed to the bilingualism.

Two German words beginning with the cluster [ts], $z u$ and Zunge, were attempted. The affricate was not handled successfully, which might be thought to be due to the fact that English does not use it initially. But consonant clusters were not mastered at this stage anyway; the same affricate medially and terminally, positions in which it is common in English, was handled just as unsuccessfully.

English long vowels, especially [e], lost their diphthongal off-glide, except in terminal position, becoming pure vowels as in German. But again, this simplification has been observed with monolingual Englishspeaking children.

Of the two typically German fricatives [c] and [ $x$, the child did not 
learn the former in the first two years and seemed to avoid words containing this common German sound. That might be due to the lack of support from English. Yet, the other fricative, $[\mathrm{x}]$, was learned in two words. More frequently, to be sure, it was omitted or replaced by the stop [k], and in many other instances when the sound occurred, it was merely a non-standard off-glide of terminal high vowels, English as well as German.

Thus the child had, by the end of the second year, a slightly wider experience with sounds than monolingual children; but they still belonged to a unified set of phonemes not differentiated by languages.

In the syntax the situation is similar. The free mixing of English and German vocabulary in many of her sentences was a conspicuous feature of her speech. But the very fact that she mixed lexical items proves that there was no real bilingualism yet. Words from the two languages did not belong to two different speech systems but to one, which was bilingual only in the sense that its morphemes came objectively from two languages. Correspondingly, the vocabulary choice was not yet determined by the person addressed; she used German words for English speakers and vice versa.

As far as syntactic structure is concerned, there was no structure during the stage of one-word utterances. The one-word stage is a long one with many children. In my case it lasted from seven to twelve months, depending on what you count as a word, and on what you count as a structured combination of two words. As soon as two words were put together in a non-enumerative fashion, the learning of syntactic patterns began. Combining a subject with a predicate (which was not necessarily based on a standard verb) was learned at the age of 1 year and 8 months. The learning of the pattern verbobject began one month later and took several months. The sophisticated combination subject-verb-object was acquired, with a heroic effort, during the last month of the second year. These patterns, along with simpler ones like adjective-noun, are however, the same in the two languages. Their learning did not require the acquisition of two separate sets of syntactic combinations.

There was one construction in which a bilingual clash could have occurred, namely the English pattern, Watch this roll, Watch me string beads, Watch me open the sandbox. This simplest form of a complex 
sentence, in which the subordination of the second clause has almost no formal expression in the standard language, is common in English, but has only a very restricted use in German. The child began to learn this pattern during the last month of the second year, and two months later $(2 ; 1)$ it was used freely (the examples are from $2 ; 1$ ) regularly with English words, however. That does not mean that she reserved this pattern for use in English sentences; there was actually one example $(1 ; 11)$ in which a German adverb acted as the sccond predicate: Ask Mama this aus, "I am going to ask Mama to take this off". By that time her speech had become so predominantly English for a time that German words were not too likely to intrude. Thus a bilingual clash was avoided.

In the morphology there was also little occasion for bilingual interference. The child was still operating with syntactic patterns devoid of formal expression beyond word order. Nouns and verbs were used in single base forms, without distinctions as to number, person, tense, etc. The only bound morpheme which began to appear occasionally at the end of the second year was the ending [S] for possessives and plurals of nouns. For possessives the German and the English endings resulted by phonetic rules in the same substitute; it cannot be decided whether her form [mamaS] was based on English Mama's or German Mamas. For the plural the ending is definitely based on English; but it was not transferred to German nouns, and no German pattern for contrasting singular and plural was attempted. The plural category was barely beginning to be appreciated.

Summarizing this first part of the scrutiny, we find that the child was not really bilingual during the first two years. She combined two models into one speech form. No clash between two sets of patterns occurred in sounds, syntax, or morphology. Compound nouns and verbs were sometimes hybrid; but that, too, does not mean a clash of patterns; the two languages use the same patterns.

The situation changed in later years, when the child became conscious of the bilingualism which faced her, and learned to keep the two languages apart. The first signs of this consciousness came soon after her second birthday. This seems to be the normal time; it is reported by other observers, too. She commented on the language difference, the first time even with the use of the language name, German, which we had not introduced to her. Usually she would 
contrast "I say" with "you say", speaking to me. Sometimes she asked me, "How does Mama say it?"-a sly contrivance to get me to say an English word, without success. Thus, with the consciousness of bilingualism had come the principle of associating a language with a person-in her case associating German with me, the lone German speaker in her world. When she was a little over four years old, she asked, "Mother, do all fathers speak German?" This question confirms the operation of the person-language principle, together with a generalization which shows that the bilingual status of our family had previously not revealed itself to her as exceptional, and with the first doubt about the correctness of the generalization. At the same time she commented once on the fact that a radio speaker was "talking German", with pleased surprise: only fathers were expected to speak German, not radios. Just once, four weeks later, she asked me, "Papa, why is it that you speak German?" The question was not a criticism, although her own language was by that time definitely English; but it was a sign that bilingualism was now a conscious problem. At the age of six and seven she showed by occasional utterances that she was proud of her own bilingualism.

After this excursion I turn to some observations from the later stage, after the second birthday but before the interlude in Germany at age five, the stage when bilingualism had begun to be conscious, but when the child had turned rather resolutely to English. During the third year most of the remaining phonetic imperfections were ironed out and most of the missing sounds were added. For instance, the English vowel [æ] of hat began to replace the substitute [a] at $2 ; 3 ;[\Lambda]$ of hut began to be correct at $2 ; 4$, so that two new vowel phonemes were added to her stock. She was less successful in imitating German rounded front vowels; her developing English sound system overshadowed the German one. [y] was sometimes rendered by [u], frequently by [i], once decomposed into the diphthong [ur], and only occasionally imitated correctly. [ø] was correct in the last month of the third year, [y] by $3 ; 3$; but she still slipped sometimes into unrounded forms of the vowels in the fourth and fifth years. These German phonemes were not very stable because the support from English was lacking.

There is evidence that the child was beginning to separate the phonemes of German from the phonemes of English. At $3 ; 6$ she 
thought that she could make the word candle German by pronouncing it [kandl]. At 3;8 she used the English verb to hand in a German sentence, but transposed it into the form [hant]; she realized that [æ] was not a German vowel. She added immediately the question, "Is [hant] right?" It was not; the question shows that her German speech feeling was ahead of her speaking practice. She also tried pseudoGerman [blak] for English black; there were many other instances at the same time. Once she used the German word stimmen, 'to tune instruments', in English: "They are stimming", but with adapted sounds, [st] stead of [St]. She had developed "conversion patterns" (Weinreich). which proves the existence of two sets of patterns.

For consonants also the English system was perfected in the third and fourth years. [v] and [f] were learned at $2 ; 4$. Terminal voiced consonants, previously omitted or devoiced, began to be learned in the middle of the fourth year. The learning of the $t h$-sounds took until the fourth year. Consonant clusters were gradually acquired. In short, English consonants progressed slowly but steadily to adequate imitation of the standard. German consonants however were wavering. At $2 ; 4$ she achieved correct $i c h$ and ach sounds, but replaced them just as often by [k], in the same month. Later, as the English consonant system became stronger, the two German sounds became even more uncertain. They were often articulated correctly, but substitutes, which were normal English consonants, intruded again and again. There was no steady progress toward correctness as in her English. Initial [ts] as in $z u$ was still simplified to [s] in the fourth year. This may not be due to English influence; English furniture was pronounced without $[t]$ at the same time. But after she had learned the correct articulation at the beginning of the fifth year, throwbacks to the simplified [s] pronunciation continued to occur because of the stronger English speech habits.

In the syntax and morphology, the learning of English patterns progressed with astonishing rapidity early in the third year. Prepositions, articles, auxiliary verbs, all previously omitted consistently, were learned, beginning at $2 ; 3$ and $2 ; 4$. The verb acquired the ending of the third person singular and past tense forms, both weak and strong. The plurals of nouns had endings more frequently. With the increasing fluency at $2 ; 4$, clauses were combined into coherent utterances. The first co-ordinating conjunctions appeared, and practically 
at the same time she began to use a variety of complex sentences introduced by subordinating conjunctions. The English syntactic and morphological patterns, which had been very primitive at the end of the second year, were perfected rapidly.

At the same time the German syntax was stagnant. She said| only the simplest sentences, usually stereotype repetitions of a few requests. German had become decidedly recessive and needed bolstering to preserve it from extinction. Since her active German concerned mostly vocabulary items inserted into English sentences, it was not surprising that German nouns occasionally received English plural endings in the fourth year, or that she used a German plural as a singular. The principles of the several German classes of plural formation were not yet recognized. Rather is it surprising that the simple English plural form did not influence German nouns more often. The child realized that the two languages have different patterns, and while she did not master those of German, she chose to abstain from German utterances in preference to casting them into English patterns-without consistency, to be sure. There were such examples as "Kann ich haben das?"-a purely German sentence in purely English word order; or "Ich will dich zu komm rauf mit mir"-an English construction impossible in German, and with an imperative instead of an infinitive. On the other hand she did not allow German patterns to intrude into the English system. My remark, "Ich hab dich lieb", was immediately answered, "I like you too". She appreciated that the German idiomatic pattern, which she understood, had to be changed completely in English.

From the end of the fifth year the child spent half a year in Germany with us. She was left alone with German speakers for four weeks, and that time sufficed to give her complete fluency in German while English receded; she was unable to say more than a few very simple English sentences after these four weeks. However the German, while fluent, was by no means correct. Idioms and word order remained strongly influenced by English. She said "alle von diesen", which is English "all of these", not German. A "butterfly" became a "Butterfliege" instead of "Schmetterling". Instead of "nicht wahr?" she used the English idiom, "Können wir nicht?" etc. But the most conspicuous influence was in the word order, in which English and German differ greatly. At first infinitives and participles were placed too early in the 
clause, as in English; but that was corrected almost at once. She had a little more difficulty with the inversion of subject and verb after an introductory word or clause; but the wavering was overcome within a month, and inversion was mastered. The greatest difficulty was the verb-last position in subordinate clauses. It took her three months to learn, and then it became correct quite suddenly. The German pattern, unimportant to her because it was not essential for communication, had suddenly dawned on her.

Pronunciation of the few English words which she still occasionally used was quickly affected by German. After a few weeks in Germany she said "I [kant]", although she corrected it into "I [kænt]". This was of course nou a slip into a different type of English pronunciation, but an intrusion of a German vowel. At first she retained the American [r] in German words, but after about four months the German uvular [R] came in with a rush, and after six months it was used even in English words, along with American [r]. Obviously the two forms, different as their articulation is, were merely phonetic variants of the same phoneme. Her [1] was the flat German variety even in English words, and in general she pronounced English words with a decided German "accent" a month after the arrival in Germany.

Half a year's sojourn in Germany was sufficient to straighten out most of the deficiencies of her German in pronunciation and sentence structure-not quite all of them; a few English peculiarities survived the whole period, although her English was then quite inactive.

After the return to America the process of adaptation was reversed. The first day she was unable to say much in English. After a few days she could converse with her English-speaking friends. Half a month after the return she spoke English fluently again, with some German interference. After one month German and English were in balance. After four months she had some difficulty in speaking German; but after six months the reaction was overcome. Both languages were fluent; only the vocabulary was more ready at hand in English. From that time on she was really bilingual-not in the ideal sense of the word: English was much stronger; but she continued to speak both languages separately-German mostly to me, English to others.

It seemed that the features of German which she had learned last were the first to be forgotten. In the beginning German [I] and uvular [R] were still used in English and German words. But after one month 
the latter was receding even in German words; later she learned it again. A year later the rounded vowels were again often unrounded; it took years before they became stable. Immediately after the return she used German pure long vowels in English words like play. Nine months later she introduced the English diphthong into German words. At the age of seven she occasionally used [su] for $z u$ again, although by then the adaptation was exceptional. Her German sounds were less firmly anchored in her speech habits than were her English ones-except for the limited period in which German was dominant.

There were instances to show that in her bilingual state she had a good feeling for regular sound correspondences. At 6;2 she used German steif, which means "stiff", wrongly in the sense of "steep". She had transposed the [p] of the English word correctly into its normal German correspondent [f]; unfortunately German does not have such a cognate of steep. Before and after the time in Germany she often used the English word number in German adaptation, Numbern, with a German stressed vowel and plural ending; the real German word lacks the [b]. At 6;6 she called a window screen in German a [Skrin]-sound adaptation for a practically untranslatable word. A year later she used in a German sentence the mysterious word Stümpfe, which turned out to be a conversion of English stamps into German, with a most surprising use of zero grade for normal grade in the vowel.

In the word order, too, the German patterns asserted themselves in English for a while after the return from Germany. After one month she said "Then is here your school" and "When one nine years old is", although the transfer of subordinate word order into English was rare. Two months after the return: "I was earlier there" for "I was there first", German in word order and idiom. After four months in America: "Where my tooth out is, that's where I bite", again marked as very rare in my diary. On the other hand, the verb-last position of German subordinate clauses was not always correct any more after less than a month in America, under English influence, and idioms were already affected. There was clearly a struggle of patterns. However, at other times the child showed that she had a feeling for the separation of pattern sets. During the first month in Germany she had tried to translate "Ich kann dich nicht verstehen" into her atrophied English. She started: "I can't you ..." and then gave up 
-not because of a lexical deficiency, but because she felt the pattern discrepancy, which she was unable to cope with. Now, a month after the return, she said, "She wanted me to come over" and immediately translated, "Sie wollte mich $z u$..."; that was a false start; she checked herself and finished correctly, "... dass ich rüberkommen soll." With reference to her doll she said, "Die ist ja auch nicht-schlafen", but then rejected the English pattern, "is not sleeping", and corrected herself, "Die schläft auch noch nicht." During her seventh year her bilingualism became more complete. She used correct word order both in English and in German. She had learned to keep the two sets of patterns apart.

In the morphology there were a few instances in which English verbs were used with German infinitive and past participle markers, and rare examples of English nouns with German plural endings, in German contexts, which means no more than English fill-ins for lexical deficiencies, within German patterns. On the whole it is remarkable that there were so few such instances, although lexicon and idioms were the one area in which the child did not mind crossing the two languages, because no comprehensive patterns were involved. The more real bilingualism developed, the less interference of patterns was observable. Since her bilingualism was not ideal, German being decidedly the weaker half, there remained an influence of English on vocabulary, idioms, and, to a limited extent, syntax, but practically none on sounds, morphology, and word formation.

Looking back, I think I have given a picture of a mixed language, particularly during the preliminary stage when the child was still trying to build up a unified language system out of the double model. I have also outlined the struggle which led to the separation of two systems, to bilingualism. I do not know whether I have contributed much to the solution of the problems of bilingual description. It seems to me that there is a parallelism between the bilingual learning of a small child and adult bilingualism. In both cases two separate sets of patterns must be mastered, and to keep them separate means a struggle. The natural thing for both children and adults seems to be to operate with one language system, and the walls between the two systems are brittle, unless bilingualism is cultivated with effort. Then however it is possible to achieve separation; I think the resultant broadening of the linguistic base leads to an enrichment of the personality. 


\section{The Phonemes of Acadian French}

\section{ERNEST F. HADEN}

The University of Texas

Acadian French or Franco-Acadian is a variety of the French language spoken by the descendants of colonists who migrated, beginning in 1604, from France and settled first in Nova Scotia, at Port Royal, now called Annapolis Royal. The speakers of Franco-Acadian are now scattered over the area of the three Maritime Provinces of Canada, and the Madeleine Islands (see map). But they live in regions which are separated from each other by territory where English is spoken.

The historical events which led up to this present distribution of the Acadian population are so well known that only brief mention of them is called for here.

Throughout the 17th century France and England contested over the possession of these territories and their rights to settle them. Finally, in 1713, the Treaty of Utrecht ceded the ill-defined territory of New France to England. But this settled the matter only at the highest diplomatic levels. In the American colonies the struggle went on. And it seems clear that it was not principally a struggle of a political nature, involving allegiance or non-allegiance. This was merely the pretext: the struggle was probably inspired by economic competition, and religious enmity.

In any event in 1755 the Acadians were expelled from what is now Nova Scotia; some were deported; some were driven into the wilds; some died, and some found refuge in the islands of the Gulf of St. Lawrence. An estimate of their numbers in that year has been set at $14,000.1$

The Treaty of Paris, in 1763, put an end to the Seven Years' War. Many of those Acadians who were not too far away came back to Nova Scotia to try again. Pubnico Bay, east of Yarmouth, and St. Mary's Bay, north of there, received the first contingents. The eastern part of the peninsula, and Cape Breton Island received others in large numbers. From the latter resettlements they have spread thinly throughout the province. And since that period they have been industrious and fruitful, so that today their numbers amount to perhaps a quarter of a million.

${ }^{1}$ Emile Lauvrière, La tragédie d'un peuple. Paris (1923), 1.511. 


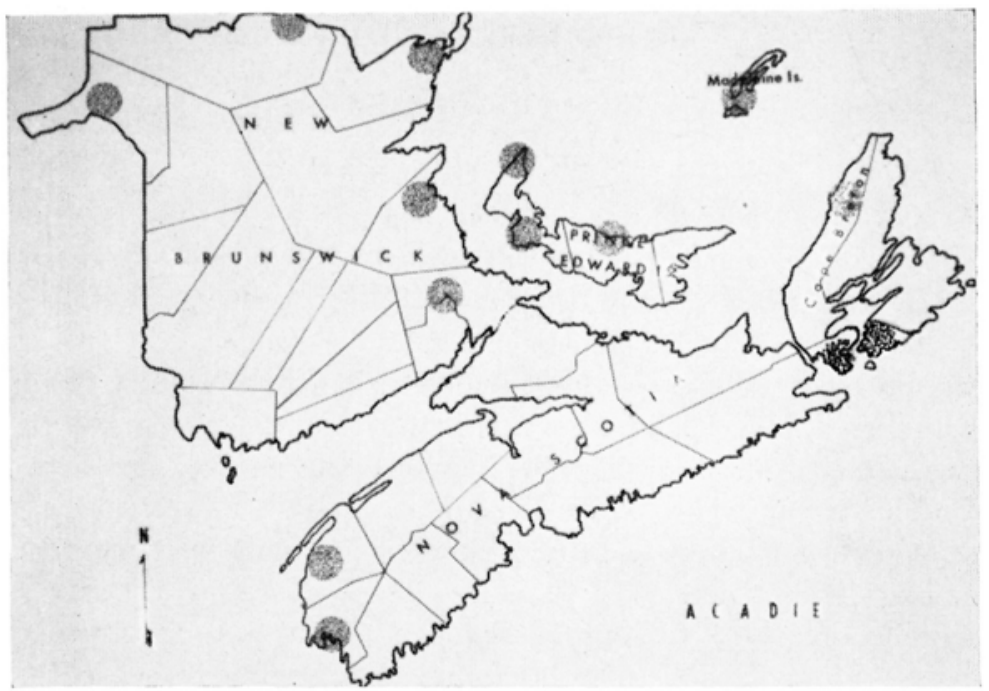

Map: Speakers of Franco-Acadian (shaded areas)

The field-work on which my data are based was done in the summer months of 1940 and 1941 with financial assistance from the Carnegie Corporation of New York. It is a pleasant duty to acknowledge here this support.

Preliminary to setting out on the actual field-work the distribution of the population speaking French was investigated by means of the then most recent census, that of 1931 . Here the population had been classified according to racial origin. It was believed, and later found to be true, that practically all who were counted as being of French racial origin, were also speakers of French. When the census of 1941 appeared, it showed, not racial origin, but a classification by languages spoken: those who spoke French only, those who spoke English only, those who spoke both these languages, and those who spoke other languages than these two. A check of the percentages of the total population in the two censuses revealed the following:

The percentage of residents of French origin in 1931 is practically the same as the percentage, in the 1941 census, of residents speaking French, either as their only language or with the addition of English. In other words: French monolinguals plus bilinguals in 1941, make up a percentage of the whole, approximately the same as the percentage 
of residents of French origin in 1931. It will be noted that the implication is pretty strong that the bilinguals among the population are those who possess French first and learn English as a second language. This being so, Franco-Acadian is spoken mainly by bilinguals.

My corpus of materials includes field-notes from a score of localities, plus a number of phonographic recordings of speech and a few songs. Some of those who were recorded were among the persons who served also as informants.

The detailed analysis of the phonemic systems in these several idiolects was the subject, a couple of years ago, of a doctoral dissertation at the University of Texas by John E. Garner. He has been teaching at the University of Texas and is transferring shortly to the Air University, Maxwell AFB, Alabama.

From this study it appears that there is enough uniformity among the several idiolects to justify the term of Acadian dialect, as distinct, for instance, from the Canadien dialect or Franco-Canadian.

The following table shows in schematic arrangement a summary inventory of the vowel phonemes of Franco-Acadian. Of course this does not mean that each idiolect has this set of vowel phonemes. Rather it is to be noted that regionally certain traits can be specified: In the northern-most localities, right across the top of the map, the front spread vowel phonemes are /i e $\varepsilon$ a/ while the rest of FrancoAcadian territory shows a fifth phoneme in this front spread series, namely:/æa/.

The nasal vowel phonemes have been treated as separate phonemes, on a par with the oral vowels, rather than as oral vowels plus a phonemic feature of nasality.

If we take the idiolect of Pointe du Sault, which represents quite well the whole region including the counties of Yarmouth and Digby, we find the following situation with regard to nasal vowel phonemes. (It will not be possible to give here more than a summary of the analytical procedure, but the matter will be treated more fully in an article which Garner is in process of writing.)

Mlle Geneviève Massignon has called attention to the diphthongization of nasal vowels in the French of southern Nova Scotia. ${ }^{2}$ Diphthongization is noticeable in final, unchecked, stressed vowels,

2 G. Massignon "Le traitement des voyelles nasales finales dans les parlers français du sud de la Nouvelle Ecosse (Canada)," Bull. de la Société de linguistique de Paris, 45.129-134 (1949). 
oral as well as nasal. We exclude then from detailed consideration here all the cases of stressed vowels in checked position, whether the checking consonant is one of the nasal consonants: $/ \mathrm{m} \mathrm{n} \mathrm{n} /$, or a non-nasal consonant.

These are excluded from the discussion because 1) forms like [grã:z]'barn', [lẽ:z] 'clothes', [õ:z] 'eleven' do not show the diphthongization which concerns us here; and 2) we have free variation in forms like the word for 'oak': [ $\left.\int \varepsilon: n\right]$ with [ $\left.\int \bar{\varepsilon}: n\right]$, and for 'lobster pot' $[\mathrm{bu} \cdot \mathrm{n}] \sim[\mathrm{bu} \cdot \mathrm{n}]$. The nasality of the vowel is found to be noncontrastive. To restate it: simple vowels, nasalized and checked by nasal /m $\mathrm{n} \mathrm{n} /$ are allophones of the corresponding non-nasal vowel phonemes.

This leaves the cases of the unchecked final nasal vowels, or those checked by velar $/ \mathrm{g} /$. The phoneme $/ \mathrm{y} /$, of limited occurrence, contrasts, in onesingleform, with $/ \mathrm{n} /$ or with $/ \mathrm{n} /$ as follows: [sprin]/[rasin], [lin].

Other occurrences of a phone [n] are limited to forms in which the

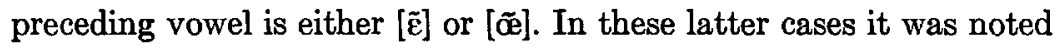
with a different symbol to indicate unreleased articulation, weakly articulated closure.

The nasal vowel phonemes are these four: $/ \tilde{\varepsilon}^{0} /, / \tilde{a}^{j} /,{ }^{3} / \tilde{e}^{0} /$ and $/ \tilde{\mathrm{u}}^{\mathrm{n}} / .^{4}$ These symbols are adopted here because they show up some special features of the idiolect of Pointe du Sault. The form [ [ $\left.\tilde{\varepsilon}^{\mathbb{D}}\right]$ is most frequently found in the stressed syllable, i.e., in phrase-final position. Its variants are the following: [ã], rarely in stressed syllable, but exclusive in unstressed syllable, and in stressed and checked syl-

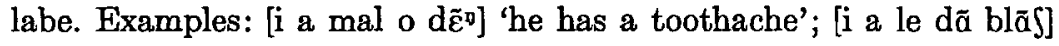
'he has white teeth'. The phoneme $/ \tilde{\varepsilon}^{\circ} /$, then, has two allophones $\left[\tilde{\varepsilon}^{p}\right]$ and $[\tilde{\alpha}]$.

The next nasal vowel phoneme is designated by $/ \tilde{a}^{\mathfrak{j}} /$, again in recognition of its most frequent manifestation. Variants in phrase-final

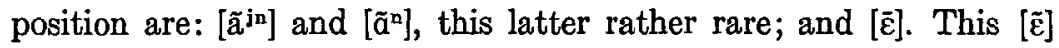
is also the exclusive representative of this phoneme group in unstressed or checked position. Examples, quoted from the field-notes:

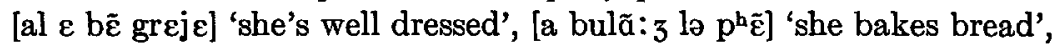
[zave fãa] 'I was hungry', [ze mãz $\varepsilon$ dy $\overline{p a}^{j}$ ] 'I ate some bread'.

3 Superior $\mathbf{j}$ stands for superior $\tilde{j}$.

4 Superior $n$ stands for superior $n$. 
The third phoneme of this type is / $\mathrm{e}^{\mathrm{o}} /$. Less frequent variants are

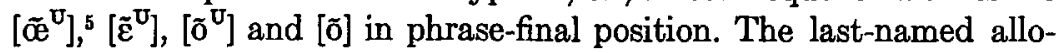
phone also occurs in unstressed position and in checked syllable. There is only one case recorded of an apparent exception to this last statement: the word [t$\tilde{\varepsilon}^{\mathrm{D}} \mathrm{dr}$ ] 'tinder', with nasal diphthong in a checked syllable.

Examples, quoted from the questionnaire: (lo sarkl $\varepsilon$ rö $^{\mathrm{p}}$ ] the hoop is round', [la ru $\varepsilon$ rõ:d] 'the wheel is round', [s mod la a Sãte yn

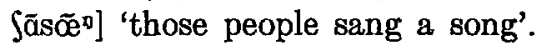

The last of these nasal vowel phonemes is $/ \tilde{\mathbf{u}}^{\mathrm{n}} /$. This is found to contrast with /un/ in the minimal pair: [pūn] 'not' [pũn] 'fist'. As has been stated, the latter form is analysed as /pun/. Allophones of lesser frequency in the phoneme-group designated by $/ \bar{u}^{\mathrm{n}} /$ are $/ \overline{\mathbf{u}}^{\mathrm{j}}$ ] in stressed position, and $[w \bar{\varepsilon}]$ in unstressed. Examples:

[de pum ki n sə gard pü̃] 'apples that don't keep', [nuzõnavố pwẽ boku] 'we don't have many'.

In all the forms which have been cited as variants of so-called nasal vowel phonemes, although they had a consonantal element following the vowel element, that consonant was recorded with a special symbol, different from the one used where the final consonant is a representative of a consonant phoneme. In other words the phonetic data separate [n] from [n] and [y] from [o]. The second in each pair is unreleased or weakly articulated. The phonemic analysis reveals the functional significance of this articulatory differentiation.

The complexity of the situation in the area of nasal vowels can be seen as so special that little is to be gained, if indeed it were possible to do, by classing the nasal vowels as oral vowels plus a feature of nasality.

Another problem, this time dealing with consonants, should be mentioned here. Once more Pointe du Sault furnishes the problem, and Caraquet, N. B. at the northern limit almost of our territory, a parallel problem. The solution of these two problems may be of some interest.

The inventory of consonant phonemes in the speech of Pointe du Sault and surrounding territory includes $/ c /$ and $/ \hat{\jmath} /$. The occurrences of $/ c /$ in the questionnaire-responses amount to twenty-three items. Some of them are repeated up to four times. In initial position,

Superior 0 stands for superior $\tilde{u}$. 
thirteen items:

[ci] 'who'

[ciskivẽ] 'who's coming?'

[cœ] 'tail'

[i cẽ d sa me:r] 'he looks like his mother'

[cẽ:z] 'fifteen'

[ce] 'wharf'

[ced] 'warm'

[cui:r] 'leather, to cook'

[cqizin] 'kitchen' (varies with [kuizin])

[cyje.r] 'spoon'

[cylot] 'trousers'

[cyi:r] 'to pick, pluck'

[cœdzõ̃ j̃] 'some (people)'

[c ${ }_{\mathfrak{c}}^{\varepsilon} l \varnothing:$ rtytkuS] 'What time do you go to bed?'

In medial position, eight items:

[mace:r] 'pus, matter'

[buc $\varepsilon_{\mathfrak{O}}^{\varepsilon d} \mathrm{~d}$ fle'r] 'bunch of flowers'

[loce] 'bolt (of a lock)'

[ecym] 'foam'

[harce:r] 'garters'

[hacet] 'hatchet'

[wace] 'to watch'

[orcórd] 'orchard'

To these must be added:

[kokro:c] 'cockroach'

[bonc] 'bunch of straw'

This last item seems to contrast in the field-notes with [lonf] 'lunch'.

/c/ occurs then in all positions. Variants of [cuizin] and [k'yizin] with aspirated [ $\left.k^{\prime}\right]$ were recorded. This is the only evidence of free variation between initial [c] and [k']. Further, the phonemes $/ k /$ and $/ \mathrm{c} /$ are in complementary distribution initially: before low central and back vowels we find $/ \mathrm{k} /$; before front vowels, spread or rounded, we find $/ c /$.

But in medial position such contrasting forms as [Jaket] 'jacket' [hacet] 'hatchet', [s Soke] 'to get angry' [wace] 'to stare at, watch', show that $/ \mathrm{k} /$ is a separate phoneme from $/ \mathrm{c} /$.

It is to be noted that some of these same lexical items were recorded 
in many other localities in. Acadia. A comparison of the variant responses in the whole area shows the following: /c/ in this idiolect has parallels in other idiolects of three kinds: [k], [tj], [c].

\begin{tabular}{|c|c|c|}
\hline$[c \varepsilon d]^{6}$ & 'warm' & [ced] \\
\hline$[c \bar{\varepsilon}: z]$ & 'fifteen' & {$[\mathrm{k} \tilde{\varepsilon}: \mathrm{z}]$} \\
\hline [cylot] & 'trousers' & [tyylot] [kylot] \\
\hline [mace:r] & 'pus' & [matje:r] \\
\hline [loce $]$ & 'bolt' & [lokg $\varepsilon]$ \\
\hline [hacet] & 'hatchet' & [hacet] \\
\hline
\end{tabular}

Caraquet, N. B. is a small town in the northeast corner of the province. It lives by fishing and lumbering. I have chosen to examine some of the data from there because it is at almost the greatest distance, physically, from Pointe du Sault, but also because it offers a problem in analysis which is of some interest.

The consonant system of the Franco-Acadian of Caraquet is as follows:

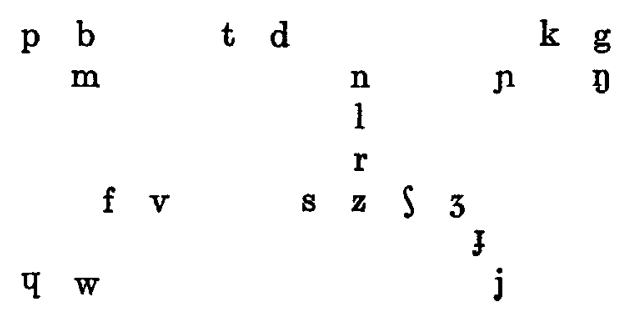

Note the presence of a phoneme $/ \hat{y} /$, voiced palatal affricate, but no $* / \mathrm{c} /$. And $/ \mathrm{f} /$ occurs in only one word in the field-notes. It is the word [ji:], the call to a horse you are driving, to get him to turn right. But, if an exhaustive vocabulary were established for this idiolect, how many other occurrences of $/ \hat{\mathrm{f}} /$ would we encounter?

${ }^{6}$ By way of digression the cognate words in Standard French may be noted, if only to demonstrate that they throw no light on the structural features of Pointe du Sault, or any other Acadian idiolect. There appear below the Standard French words which are cognates of the respective Acadian forms above.

$\begin{array}{ll}\text { [tjed] } & \text { 'tiède' } \\ \text { [kẼ:z] } & \text { 'quinze' } \\ \text { [kylot] } & \text { 'culotte' } \\ \text { [matje:R] } & \text { 'matière' } \\ \text { [loke] } & \text { 'loquet' } \\ \text { [aSet] } & \text { 'hachette' }\end{array}$


Now in Pointe du Sault, we find the phoneme $/ \hat{\mathrm{f}} /$ in initial and medial positions in some eleven items (including the same command to a horse [fi:]. This form is used in this meaning everywhere throughout the area.) The other ten are:

[jaket] 'jacket'

[ĵl] 'mouth of an animal'

[jeri] 'restored to health'

[jo:b] 'devil'

[f̂̃zble] 'gimlet'

[ĵ̣.p] 'wasp'

[ij̣œetso nami] 'he's expecting his friend'

[Sôे $\varepsilon: r]$ 'kettle'

[brajet] 'fly (of trousers)'

[ãjij] 'eel'

Some of these same lexical items turn up in Caraquet with either [g] or [j]. Thus: the word for 'wasp' is [gep] [jep]; for 'honeybee', [gep a mjel] [jep a mjel]; for 'animal's snout', [gœl] [jœl]. In addition the word for 'vegetables' is [legym] $\sim$ [lejym].

It looks like free variation between $/ g /$ and $/ \mathrm{j} /$, at least before front vowels. But, just a few more examples will destroy that theory: [pulaje] 'chicken coop' has no variant form $*$ [pulage] so far as I know; [in-avejyn] 'there was one (f. pron.)': I heard the form [jyn] many, many times, but never pronounced $*[\mathrm{gyn}]$. And the word meaning 'sty on the eyelid' was given as [orgejø]. Think what free variation might do to this form: *[orj $\varepsilon j \varnothing]$, *[orgeg $\varnothing]$.

There is then a phoneme $/ \mathrm{j} /$ with normal distribution. Allophones of this phoneme are apparently not substitutable by $[\mathrm{g}]$. But there is a phone [j] which is attested as varying with [g] before certain front vowels. Indeed the contrast between these two phonemes is demonstrated by such pairs as: [gã] 'glove' [jã] 'one (pronoun)'; [vine.g] 'vinegar' [vjej] 'old (f.)'; [magane] 'weakened (of a horse)' [mujase] 'drizzle'.

It goes without saying that all segments [j] belong to the phoneme $/ j /$. All segments [g] belong to the phoneme $/ g /$. To account for the facts exhibited by the usage in this community, we must draw up, as part of the lexicon, a list, as complete as possible, of those words with [g] which have synonymous variants with a [j] pronunciation.

It is regrettable that my data do not permit more than a very short 
list of this kind. But it is clear also that a geographical survey which merely samples each of several idiolects always leaves such gaps.

In Caraquet speech there is one other point which calls for comment here: While there is no phoneme $* / \mathrm{c} /$ in the sound-system, some of the words which elsewhere have /c/, here turn up with a voiceless prepalatal affricate, [t], or a voiceless mid- or post-palatal affricate $[\mathrm{k}]$, or a voiceless post-palatal stop [k].

The following variant forms are recorded in the field-notes:

a) in initial position, before front vowels:

[kyje:r] [tyje:r] 'spoon'

[kylot] $\sim$ [kylot] 'trousers'

[kuir] [tyii:r] 'leather'

$[\mathrm{k} \tilde{\varepsilon}: \mathrm{z}] \sim[\mathrm{t}, \tilde{\varepsilon}: \mathrm{z}]$ 'ffifteen'

Though we have no attested variants, these forms were noted, and are grouped together here to show the relationship which exists among the three phones in question:

a) initial:

[kœ:r] 'heart'

[kœlœr kile] 'What time is it?'

[ţœkzōe] 'some, a few'

[ţøk] 'some (adj)'

$[\mathrm{k} \varnothing]$ 'tail'

[ţz: dr] 'to hold'

b) medial, intervocalic:

[zuke] 'to roost'

[rëţe] 'spine'

[take] 'lock of a door'

[suţẽ:dr] 'to support'

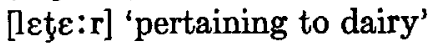

[raket] 'snowshoes'

c) medial, postconsonantal:

[virbərkš̃] 'brace and bit'

[3arķ $\varepsilon: r]$ 'garters'

These three phones [k-] [k-] [t-] seem to vary freely before front vowels, and can be assigned to the phoneme $/ \mathrm{k} /$.

Furthermore, there seems to be contrast between the [t] phone and the voiceless dental stop [t]. For example, [ote] 'to take off' contrasts with [rẽţe] 'spine'; [otel] 'hotel' with [leţe:r] 'of a dairy'; [ty] 'you' 
contrasts with [tyje:r] 'spoon'. In addition to these examples, both $[\mathrm{t}]$ and $[\mathrm{k}]$ occur before open and back vowels and certain consonants, as well as in final position.

Perhaps the most enlightening examples are the following which match up the forms from Pointe du Sault with their translations, so to speak, in the speech of Caraquet:
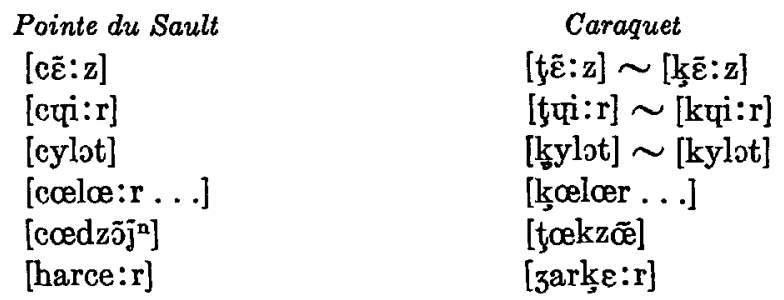

Again, it seems too bad that such a comparative list must be so short. But, even with such obvious gaps in the vocabulary, a sampling of this kind can give a fair idea of the phonemic structure of the dialect, and the main variations within that structure.

\section{Linguistic Convergence in Immigrant America}

\section{URIEL WEINREICH}

\section{Columbia University}

The fundamental contribution of structuralist thinking to historical linguistics is the theory that the patterns of a language themselves determine, at least in part, the direction in which the language changes. Stated in its simplest terms, the theory holds that a language always contains certain uneconomical imperfections which can be, and often are, eliminated by change; and the improvement of the linguistic system can be effected either by original innovation or by the adoption of elements from any accessible outside source. Thus structural diachronicists in the early days of the discipline made the assertion that a language accepts from other languages only elements which are amenable to its own structure, that is, which "correspond to its own tendencies of development," or to its "drift," as Sapir would put it. Accordingly, every language can be regarded as con- 
stantly exposed to potential interference from its neighboring languages, and convergence between unrelated languages becomes a perfectly understandable phenomenon.

Today the structural point of view in diachronic research is modified on two counts, at least. First, it is realized that the socio-cultural setting of language contact-factors of "prestige" and the likehas to be considered along with structural factors in an explanation of linguistic change and borrowing (which is really but a special case of change). Secondly, care must be taken to avoid a vicious circle like the following: A change took place or a foreign element was selected for adoption because there had been a structural need for the change or the adoption. How can we be sure that the need existed? Because the change or the adoption was made. If such an argument is admitted, the structural point of view becomes dogmatic, i.e. not subject to proof by facts.

I need not dwell here on the general aspect of these fundamental problems of historical linguisties; they have been and are being treated in detail by various American and European theoreticians. I should like to restrict myself to that special case of change in language which is interlingual influence. In my book, Languages in Contact (1953), I suggested two directions in which studies of contact and convergence might head in order to keep away from the fatal pitfalls of dogmatism and to give due weight to socio-cultural as well as to structural factors. In contrast to many of the best existing works on convergence which were concerned with historical problems and therefore had to fill important gaps by reconstruction, I think we now need more intensive field studies in bilingualism. In observing bilingualism in the field, we can make our standards of description as strict as we like and collect all the information, linguistic and sociocultural, that may be relevant to our problem. We may compare the behavior of persons in an advanced state of bilingualism with those who are only beginning to experience contact with the other language -a comparison which usually has to be eliminated entirely in historical studies. We may thus arrive at a realistic picture of how foreign elements are introduced and resisted and how they function in the complicated framework of linguistic and extra-linguistic conditions under which all human speech takes place.

Another possibility of improving studies of language influence is to 
investigate situations of multiple contact. By this I mean the contact of the same language with two or more others. The advantages of this approach are obvious. If the structure of a language is, as is asserted, partly responsible for the selective resistance to and acceptance of outside influence, then it should be possible in a multiple contact situation to test the reaction of several different "recipient" structures to the same source of borrowings, and vice versa.

One area which offers good opportunities for refined study of language influence is the United States. Here a variety of languages are in contact with a relatively uniform version of English. We have a common denominator on one side, and on the other, a wide range of structures in the immigrant and native Indian languages as different as Chinese and Lithuanian or Taos and Kutenai. Provided we ask the right questions, we may observe the handling of English elements adopted by the many languages and, on the other hand, the handling by bilingual English speakers of elements taken over from these various languages. Fortunately the contacts with English are in many cases still very much alive and are therefore directly accessible to first-hand description by the methods of our choosing.

There is one important difference between the United States of the twentieth century and many of the previously studied areas of convergence. In the schoolbook examples, such as the Balkans or the Flemish-Walloon region, there is a language border, where the bilinguals live, and a unilingual hinterland into which the innovations of the border area "trickle down." In Switzerland, for example, interesting observations have been made on how the rate of "trickling" of influence is reduced as one gets further away from the language border. In the United States of today, the same factors which have turned the country almost into a single (though not necessarily uniform) dialectological community-city living, motor transportation, and above all mass media of communication-have reduced the space factor in interlingual contacts nearly to zero. Language borders are virtually non-existent; the English area is superimposed on the others. On the non-English side of the fence, there is practically no difference between persons living close to or far from the line of contact. On the English side, geographic distance does survive, at least as a rudimentary factor; for example, the extent to which Pennsylvania German influences your English probably does depend on whether 
you do or do not live in, say, Lehigh County, Pennsylvania. But certain interesting "extra-geographic" channels of diffusion have also been established on the English side of the contact. The reduplicative shm-device (handsome-shmandsome), of Yiddish origin, can be diffused to the whole country simultaneously by a popular TV comedian who made his beginnings on the Yiddish stage, just as a new toothpaste is not necessarily diffused first in the vicinity of the factory which makes it. Yet, despite the large-scale elimination of the space factor and the rise of novel diffusion channels, the multiple language contacts of this country do contain rich sources of information on influence and convergence as universal processes.

In my discussion I would like, for reasons of space, to eliminate the problem of the native Indian languages and to skip over the impact of the immigrant languages on English, devoting myself instead to the influence that English in turn has exerted on the immigrant languages. A great deal of work has already been done in this field; a bibliography on the subject includes many dozens of items. They range from one-paragraph communications in journals like American Speech, through stimulating but often inaccurate and condescending surveys like Mencken's in the appendix to The American Language, to an exemplary two-volume monograph like Einar Haugen's The Norwegian Language in America (1953). Recent scholars with general linguistic interests like Haugen and Leo Pap (Portuguese-American Speech, 1949) have even taken advantage of previous studies by occasionally comparing the effects of English on various languages. But they could not go very far in this direction because the existing studies are, for many reasons, not really comparable. (1) Some used evidence of the spoken languagd while others, like Witold Doroszewski's valuable analysis of American Polish, based themselves principally on the language of the press and of popular literature. (2) Not all studies made use of the same descriptive methods, and many were not made by trained linguists at all. (3) Even where the descriptive methods are adequate, the classification of the material is not uniform. It was not until last year that Haugen proposed a foolproof classification of loanwords according to the mechanism of influence involved. But if we now want to study comparatively what Haugen calls "homophonous loan-shift extensions," we could not readily locate them in an old list of, say, Ameri- 
can Greek vocabulary. (4) Few or no studies have attempted to be exhaustive. To be sure, completeness in the treatment of vocabulary, especially the vocabulary of languages as unstandardized as those of immigrants to the United States, is unattainable. But we could wish for a uniform and exhaustive coverage of at least a set of selected questions for all the languages involved.

I should like to delineate some of the comparative problems that could, I think, be solved if the data were collected according to a coordinated plan. In referring to such languages as Amer. Norwegian or Amer. Italian, I have in mind, of course, their unstandardized forms.

We might begin with some lexical problems. Faced with lists of thousands of English loanwords in any immigrant language, we are apt to lose sight of the very important fact that not everything is borrowed. In Amer. Yiddish, for example, walk is a favorite loanword, both as a noun and as a verb; but talk is never borrowed. Similarly, for reasons which remain a mystery, butter is borrowed but bread is not. Such statements apply, of course, only to one language at a time. The question then arises: Do the various immigrants borrow and refrain from borrowing the same English words? Our sources cite bad, for example, as a loanword in Amer. Portuguese and Amer. Norwegian, but not in Amer. Yiddish. What about Amer. Chinese, Amer. Polish, and so forth? Does the absence of bad in a list of Amer. Yiddish loanwords mean that it does not occur in that language, or that the compiler of the Yiddish list happened not to note it? What we need is a standard checklist for all immigrant languages. A step in this direction has already been taken by Sandra M. Schor, a student at Columbia University, who is completing a cumulative vocabulary of English loanwords in immigrant languages based on published material. Field investigation of all gaps turned up by her vocabulary will be in order next.

Since a comparative loanword dictionary of the immigrant languages could lead to important cultural inferences, some thought should be given to the semantic grouping of the loanwords. In practice up to now, every student of loanwords has made his own grouping on an ad-hoc basis, and no two are the same. Haugen, for example, has a highly readable account of loanword fields with these chapter headings: Official Life, Economic Pursuits, Social Experiences, and 
General Terms. Unnumbered paragraphs within his second chapter set off subtopics such as farm structures, plants, house plan and furnishings, heat and light, dress, and so forth. Pap, in his Amer. Portuguese study, has paragraphs on food terms, meals, clothing, home furnishings, house plan, the human body and its functions, etc. I am afraid that such informal groupings will fail us in our comparative needs. Is it valid, for example, to say that English expressions "did not affect the immigrant's emotional and general behavior to the same extent as his economic life"? With a view to the socio-cultural value of such lists, we linguists could use some outside advice from the social sciences, I think, since it clearly won't do to classify any behavior as miscellaneous or even "general".

A further delicate point is the fate of the native words with which the loanwords come into competition. The old word may be displaced completely, as Norwegian kli 'coarse flour' was displaced by bran; it may become specialized in its reference, as happened in Amer. Norwegian where the loanword beer has come to stand for the beverage bought at the store, while the native $\phi l$ refers rather to home brew. As often as not there is complete confusion of the terms; speakers of Amer. Norwegian seem to alternate between borrowed dipo and native stasjon for 'depot'. The fate of the old words is one aspect of linguistic interference and cannot be left out of the study. But the problem is delicate because direct inquiry yields the required information only in particularly favorable cases.

The mechanisms of lexical interference are several. Words may be transferred outright, or there may be so-called loan translations, or a blend of the two processes may take place. It is a well known fact that some languages have chanelled all their borrowing into the loantranslation field; this is true, for instance, of Icelandic as contrasted with Modern English, of Polish as contrasted with Russian, and so on. But the evidence on record concerns only these highly standardized languages. We need information as to whether languages that have been less subject to centralized regulation display similar preferences. The wide range of American immigrant languages can provide a storehouse of data. On some points of detail, the differential reaction of the languages is self-explanatory. The Germanic immigrant languages, for example, have been far more liable than the Romance tongues to loan-translate English complemented verbs of 
the type read up (on something)-cf. Penna. German uf-lese. On the other hand, it is the Romance languages which have been quick to adjust the meaning of words like French introduire or Italian introdurre to that of the English cognate introduce 'to acquaint'. Other interesting differences emerge which are harder to explain. Haugen finds, for instance, that Amer. Norwegian has few loan translations of phrases comparable to Amer. Portuguese correr para mayor 'to run for mayor' or Amer. Yiddish ton mayn best 'to do my best'. Clearly we need comparable data in far greater abundance before generalizations and explanations can be hazarded.

Let us consider some grammatical problems. A favorite question in bilingualism study deals with the assignment of vocabulary to a conventional grammatical class in the "recipient" language. Thus the verb ring is in Amer. Yiddish conjugated as a strong vert (ringengerungen), while catch is inflected as a regular verb (ketshn-geketsht). Train is adopted as a feminine noun (di treyn) while (news)paper becomes masculine (der peyper) and pencil is neuter (dos pensl). Various factors have been adduced by the many writers on the problem to account for the vagaries of grammatical classification in the recipient languages. Without going into the question further, it is safe to say that far more data from many different languages will be needed before the problem can approach a solution.

Another major problem concerns the impact of the English grammatical pattern on the immigrant language. The syntax of all immigrant languages is full of Anglicisms. In Texas German, for example, an incipient loss of distinction between dative and accusative has been attributed to English influence. Emphatic formulas beginning this is what... are often duplicated in Amer. German (das ist was ...). The sequence of tenses of English has not been without widespread effect. The truly enviable English system of noun compounding has played havoc with immigrant patterns: Amer. Portuguese speakers have formed a Português Recreativo Club; speakers of Amer. Yiddish have broken up the proper stress relationships of compounds by creating forms like árbeter ring (instead of árbeter-ring) on the model of 'Wórkmen's Círcle'. What we need is a point-by-point juxtaposition of the grammatical systems in contact with English, a delimitation of the areas of likely interference, and a directed search for instances of such interference. 
A hotly debated point is the possibility of an outright transfer of bound morphemes, especially inflectional affixes, from one language to another. Can we find an Amer. Italian or Amer. Russian noun of native origin carrying an -s-plural? $\mathrm{My}$ own experience leads me to suggest that such forms do occur in the actual speech of bilinguals, but they are surely evanescent and are often corrected by the speaker on the spot. Mechanically recorded conversations between immigrant bilinguals may disclose these and many other phenomena which interviews with single informants would not bring forth.

We come now to interference in sounds. The several possibilities of what is generally referred to as sound substitution have been elaborately worked out. Since linguists disagree on matters of phonemic theory, their formulations of sound interference are also different; but the explanation of the facts is hardly controversial. It may be useful to point out that the investigation of prosodic interference is far behind that of the so-called segmental phonemes. Despite the advances made in analyzing and predicting prosodic difficulties during the preparation of spoken English courses for foreigners, more descriptive work remains to be done. A foreigner, as we know, who has spent even a few years in the United States can be spotted by his European countrymen even if he does not use a single lexical, grammatical, or segment-phonemic Anglicism. It is the elusive impact of English prosody, yet to be described, which apparently gives him away.

If we should succeed in collecting comparable data in great abundance on the preceding and related problems of lexical, grammatical, and phonological interference, we may at last find it possible to test some hypotheses concerning the role of linguistic structure as a regulator or selector of interference effects in a contact situation. But we take it for granted that the role of structure can become apparent only under similar socio-cultural conditions of contact, i.e. other things being equal. Alas, other things are almost never equal. In the first place, the acculturated American community does not look back on all immigrant groups with the same attitude. A French accent is in many circles an asset. A German accent may be tolerated or even go unnoticed in some regions where a Polish accent would stick out like a sore thumb. Secondly, among the immigrants themselves attitudes toward the native language differ. Some groups come with 
pride in their language, others with deep feelings of inferiority. Thirdly, the differences in the level of old-country education of the individual immigrant and his attitude to his native language form a familiar correlation which probably cuts straight across the various ethnic groups. The individual with the more cultivated view of his own language will probably make a greater effort to resist the inroads of English."But even on this point considerations of religion, urbanization, and the often inconstant political relations between the United States and the homeland of the immigrant may do much to upset a facilely erected scheme.

When "other things" are not equal, the only alternative is to neutralize the difference by making proper allowances for them. This means that linguists cannot hope to do justice by themselves to a comparative study of language contact in America. There is a definite need for historical, sociological and anthropological consultation-in short, for interdisciplinary teamwork.

Unequalled research opportunities are afforded by the comparative study of immigrant languages for helping to solve some of the most timely and fascinating questions of linguistic convergence, and more generally, of linguistic change. So far, however, the lot of these studies has not been a happy one. Interest in the history of immigration has not been overly enthusiastic; on the linguistic side, the commendable beginning made by the Committee on American Speech of the American Council of Learned Societies in calling the 1940 Conference on non-English speech unfortunately remained without systematic results. Perhaps now is a good time to renew the call for research in this field. The scholarly interest in the effects of bilingualism on language is increasing; this very panel is one of its symptoms. Immigration history is also giving signs of increased respectability; it may not be idle to hope for some moral support from a sister discipline.

The ideal would be a central institution from which comparative research could proceed. A university with a linguistics curriculum which would be given the means to attract qualified students (perhaps themselves of immigrant backgrounds) and to apportion the field among them would, I think, be the best way of launching such a program. But if this is too ambitious a plan, a body of specialists should at least work out a well-rounded and pilot-tested research 
program specifying the information we need about every language. Interested individuals could then obtain and submit the required data as the opportunity allows. Their efforts would at least have the fullest cumulative value, even if they should have to remain scattered in time and place.

\section{Discussion}

In the discussion which followed the First Session J. P. VINAY (University of Montreal) made some additional remarks on bilingual-. ism:

I have lived practically all my life in bilingual countries and I would like to point out two phenomena which might be of interest in this connection. The first one is the fact that speakers of two languages very often shift from one language to another without any apparent reason. It is a kind of free variation. For instance, I lived in Wales for a long time and I found that people who speak Welsh will suddenly change into English either for a short or a long sentence. I did not see any reason why they did so, but I find the same phenomenon in French Canada, where I live now. Two French Canadians talking to each other will suddenly switch into English. I assume that there may be something in that particular sentence or idiom that is unacceptable to the structure of the other language.

My second point is that it seems to me that many constructions which are not acceptable from the point of view of syntax, may become acceptable on another level. For instance, I have never seen or heard any phrase in. French Canadian which attempts to imitate the -ing form in English, as je suis venant, je suis fumant. This has never been done to my knowledge. It would be extremely contrary to the structure of French. On the other hand, on the lexical level, I have often heard and seen patterns which are obviously not French, but which have been accepted. I have seen one recently in French literature, even printed in France. The word science-fiction is obviously something taken from America. It goes against the grain of all French patterns in the field of lexicology, but it is accepted now. If a good many of these words would be accepted, I submit, this would introduce a new category in French.

The same thing happened in French spoken in Metz. There they 
say Viens-tu avec? (Kommst du mit?) If many such items were adopted, this would introduce a new category into the French language.

EINAR HAUGEN (University of Wisconsin): I would point out that on page 65 of my book I have discussed the point to which you are referring. I called it a language-switch and said that speakers will often switch back and forth, quite unaware that they are doing it. They are accustomed to having bilingual speakers before them and know that whichever language they use they will be understood. $\mathrm{My}$ guess at the reason for it is similar to yours, that they anticipate something coming which they cannot say, or do not feel can be as well said in the other language-so without thinking about it, they do switch. It affects our theoretical approach to the whole thing also, because it is often said that every utterance must belong to one language. I have never been able to understand the meaning of that phrase although it is spoken by some of our finest authorities in linguistics. Whether it is true depends entirely on how you define utterance. If every single word is a single utterance, of course, it might be true because I cannot remember anyone who switched in the middle of a word, although even that could theoretically occur. But how long does an utterance have to be to validate this particular general statement? I know many cases where people switch very rapidly back and forth, and what language are they speaking in that case? They are speaking two different languages, one after the other usually.

HUGO MUELLER (Georgetown University): I would like to come back to Professor Weinreich's remark that the mere fact that this panel on bilingualism is being held shows the increasing interest in that field. One reason we in this Institute had this subject put on the panel is that the particular group of people who work here feel the impact of that problem every day. There are a number of people around here, whose native language is not English. The question comes up very often: Why is it that we have such difficulty in becoming bilingual? Why is it that even the trained linguists have such difficulty? Why is it that in spite of their ability in linguistic analysis they always keep their accent and traces of the native language? And here is where the aspect of methodology comes in. This problem interests us predominantly as a methodological prob- 
lem. I would like to ask Professor Leopold this question in regard to his case-his daughter (who, as I understand, now is married and has a child herself): Is his daughter still bilingual today, and if so, or if not, does that reflect on the validity of the often expressed statement that when starting to learn a foreign language, it is very important that the child should begin at a very early age? Is bilingualism in an individual easily attainable by an early exposure to the two languages? As far as I can see from the material that Professor Leopold has given us, she was not really bilingual, she was not easily becoming bilingual, but she was monolingual, always according to the language which was predominant in her communication situation at a particular time. Did she really become bilingual to such an extent that one could call her bilingual even now?

WERNER F. LEOPOLD: (Northwestern University): I tried to make clear that she was not bilingual in the early years. German was so much inferior in its strength to the English because of the Englishspeaking environment that she needed the reinforcement of the German by half a year in Germany. I feel that a year or two after that time she began to be really bilingual, and I repeat, not bilingual in the ideal sense. Some people define bilingualism as the ability to speak two languages equally well. I don't see that that is a necessary ingredient of the definition; to me it is sufficient that an individual be able to transact all the ordinary affairs of daily life or whatever special field is under consideration, to call that individual bilingual. I believe she is bilingual now as long as you accept the reservation that she is not ideally bilingual. Her real language is English; there is no doubt about that. Now the question of associating the language with certain persons with whom she talked is a very interesting one. I would say it is definitely advantageous to tie the language to a certain individual if you want to teach a small child two languages. It is much better that the child should get into the habit of speaking one language to one person, and another language to another person, and it did work out that way in my case. She spoke German eventually only to me, and English to practically everyone else. I don't think it is necessary, but it is a pedagogical help.

GINAR HAUGEN: We want to be sure we know what we are talking about when we say bilingualism. I just heard Professor Mueller say someone was not bilingual because you could still hear his accent. 
That I would consider so narrow a definition of bilingualism that most of us would have nobody to study if we were to limit it in that way. I have defined bilingualism in my book as including anyone who can form utterances in a second language. A person who has studied a foreign language for a week in the modern method ought to be bilingual in that sense. The alternative would be to say a person is bilingual who can be accepted in two different language environments as a native speaker. Very few people actually can.

WILLIAM E. WELMERs (Cornell): Mention has been made of the kind of limitation of bilingualism in regard to the problem of who it is to whom you are speaking. It seems to me that there is another kind of limitation that is most interesting to study, and that is the problem of what you are talking about. The case of a close friend of mine may be mentioned here-a man who came to this country from the Netherlands at the age of eight or nine. He is now completely fluent in English. He expressed himself very completely on this subject one time, so that his situation is, I think, very easy to outline. He said that when he is in the Netherlands and speaking to monolingual speakers of Dutch, he speaks Dutch with perfect freedom on any subject on which his education enables him to talk. I don't know that he could talk about cleaning a carburetor, but I am not sure that he could talk about it in English either for that matter, because he doesn't clean carburetors. However, in matters of buying and selling in a store, ordering a meal in a restaurant, or philosophy or theology, and many other subjects in between, he can talk with complete freedom in Dutch to monolingual speakers of Dutch. In speaking to monolingual speakers of English he has exactly the same freedom in English. He said the problem arises when he speaks to another person of the same background as himself, who is also bilingual in Dutch or English. Then what does he choose? He said, if he is preaching a sermon (and he is a minister) to a congregation which understands both Dutch and English, he prefers to preach in English; if he is asked to offer a prayer, he prefers to pray in English; if he is asked to talk about philosophy, he prefers to discuss philosophy in English; if he is asked to talk about almost any subject you could mention, he prefers to discuss that subject in English, but he says-and this I think is a significant addition-"when I am in my room at night, and get down on my knees to pray, Dutch is the 
language in which $I$ talk to God-it is the language $I$ learned at my mother's knee; I can't pray privately in English."

Now a comparable situation, but at another extreme of language learning, was another man who was monolingual in English until his early twenties, and then was going to go to Amsterdam to do some graduate work in theology. Three months before he left, he found himself a Dutch grammar which has been described as being more of a liability than an asset-and I agree with that description-and began to learn Dutch. He went to the Netherlands in early 1939, and was compelled to return after one semester there. In telling me some of the things he had learned, he got himself involved in new terminology, terminology that he and $I$ had not shared before, and after five minutes of discussion, he broke down completely in English and found it necessary to express himself in Dutch. His Dutch was a most marvelous thing with a wonderful Philadelphia accent, but here he was talking about a subject which he was completely unable to discuss in English; he just didn't have the vocabulary for it. So bilingualism by subject, as well as by the person to whom you are speaking, I think, is a most interesting study.

EINAR HAUGEN: There is a complete section on this subject in Weinreich's book on Languages in Contact, a specialization of language by topics and by interlocutors, which I think is quite relevant to what Professor Welmers has just said. I think we have all had that experience with bilinguals; the problem of subject matter as well as persons spoken to.

HERMAN RAMRAS (Minnesota) expressed the need for spelling out in certain places which are accessible to the public, the definition of bilingualism, "because one of the greatest difficulties we have is that by fostering certain illusions which arise from such phrases as 'speaking like a native' being bandied about, we do not accomplish things which we can accomplish; that is, to get a good many people to speak a language reasonably well, perhaps quite a few to speak it like natives if they get into the native environment and stay there for a while."

M. G. MARTÍNEz (Georgetown University) voiced his doubts as to whether any person can really master two languages. He granted the possibility of a practical command of a foreign language for everyday life, even for writing articles or books, but when it comes to the native 
accent, he thought that "to expect a bilingualist to master two languages is just a dream."

PAUL GARVIN (Georgetown University): My experience with bilingualism as a linguist has been much more limited than that of anyone on the panel, though I have had some practical experience with it as an informant. There are, however, a few things that entered my mind, as these people were talking.

First of all, in regard to Professor Weinreich's address, I think he has correctly outlined the procedure which we generally follow-that is, we go from an intuitive insight to some kind of conceptualization, and then to some technical work, and I think we now are at the stage where we have to go back from the technical to the conceptual and play back and forth until we hit a conceptualization of a somewhat more permanent nature, and it is in regard to that that I have a few comments. One is that I think a basic problem, which has been suggested in the comments of the speakers and by people in the discussions and which has not been stated too clearly, is to maintain the difference between what I would like to call co-existing patterns, and interference by one pattern upon the other. Now, for instance, the language-switching situation very clearly is an example of co-existing patterns, whereas the use of loans and loan translations is quite clearly an instance of interference, and the two things should be kept apart conceptually, although they are not very clear in many cases in the empirical situation. There is also a certain correlation between the two which I could mention from my own observations and this has to do with another concept with which I have toyed and which I would like to call "loan-proneness." That is, I have observed that in two languages with which $I$ have worked very intensively there is a distinct difference in the degree to which the speakers are able or willing to assimilate loans from other languages. In Kutenai it is exceedingly unusual to borrow English words; in Ponopean, which is spoken in an entirely different part of the world, it is quite usual to borrow words from English, and for that matter, from other languages. With the Kutenai, who speak a relatively less loan-prone language, you find that informants in spontaneous conversations switch to English with English phonemes whenever necessary, whereas Ponopeans in spontaneous conversations use English loans with Ponopean phonemes, and you can tell when you listen to a Kutenai 
stretch of speech that such and such two or three contours are not Kutenai, they are English. The man says "Judge Roth," which is a completely impossible sequence in Kutenai. It isn't perfect English, but it is his English as opposed to his Kutenai. This brings back another point which has some reference to the comment of the gentleman from Montreal, and that is, that different "layers" of linguistic structure to my mind are variously susceptible to interference; for instance, it seems to me that obviously in Kutenai the phonological layer is less susceptible than the syntactic layer; there are syntactic loans from English which break up the traditionally known Indian pattern of incorporating objects in "classical" Kutenai, if you will allow the phrase. To say such a thing as "I built a house", you use a verb with an incorporated object, but the people who are about 20 or 30 and who speak quite fluent Kutenai, do not use an incorporated object, they use a verb plus a noun meaning house, and this to my mind is a syntactic loan translation. They don't, however, borrow English phonemes readily.

This brings about another question in regard to the loans: whether or not a loan is taken over and then functions externally as a single unit while being internally structured in various ways, I think this may explain the example of "science-fiction". I have a feeling that perhaps in French-Canadian such items as "science-fiction" are simply single units, perhaps even from the standpoint of FrenchCanadian single morphemes, and therefore cannot be in any way interfering influences upon the pattern. It is a loan morpheme just like any other loan morpheme that you might find in French. This is where I would at least investigate-that is, as to whether "sciencefiction" internally is really two. elements in French-Canadian, or whether it is just one.

And the final point on which I would like to make a comment is the matter of the bilinguals who speak two languages fairly natively. I have some experience in that, not as a linguist, but as an informant, because $I$ grew up in a part of the world where there are extensive communities in which people as a matter of course speak at least two or sometimes three languages quite natively. For instance, in Slovakia all Hungarian families of my generation (and I lived in Slovakia until I was seven) as a matter of course spoke fluent Hungarian, as Hungarians in Hungary, and their children who went to 
Slovak schools would speak the same kind of Slovak as all the Slovaks. Another classical example, of course, are the Germans in Prague. And there is one problem which emerges, which to my mind has not been investigated and bears some linguistic investigation, and that is, that very impressionistically I remember that the Prague Germans had a sort of compromise phonemic pattern which was sufficiently Czech to suit the Czech and sufficiently German to suit the German. That is, there was no great difference in my impression between, for instance, the vowels they used in the two languages, and so on. At least in the allophones, there may have been phonemic differences, and a Prague German could be told from a monolingual German from another part of Bohemia, and his Czech could be told from that of a monolingual $\mathrm{Czech}$, but it was completely acceptable. I think that a kind of compromise pattern existed there, in which the two linguistic patterns were at least phonetically assimilated, and I wonder whether or not something along those lines could be observed, let us say, among Alsatians, who are bilingual (French-German), and among Belgians who are bilingual (FrenchFlemish). 


\title{
II. First Luncheon Speech
}

\section{Cultural Implications of Linguistic Science}

\author{
NORMAN A. McQUOWN
}

University of Chicago

In attempting to develop this topic, I shall put my remarks in the form of a dialogue-in question, answer, and commentary. I shall attempt to characterize the working habits of the practitioner of linguistic science and of the analyst of culture (be he humanist or social scientist), and I shall try to contrast the results of their respective labors.

A. What question does the linguist ask first? How does it work?

B. What question does the student of culture ask frst? Why is it this way?

\section{A}

I. How does the linguist go about finding the answer to his question?

a. He identifies and circumscribes the object of study, i.e. he defines, for the process under investigation, its medium.

b. He brings to bear on the object of study (the linguistic process) a preliminary frame (specifically created for the purpose). Within this frame (with built-in questions each of which requires an answer) he proceeds to describe (in a preliminary fashion) the process. The results of this procedure constitute his raw data.

c. He performs a series of operations on this raw data for the purpose of eliciting its structure (its inventory of structure points and structural relations).

d. He states the results of the analysis in a number of ways: he lists the units, he specifies the levels on which they appear, he states the ordering of units and of levels, he portrays (verbally or diagrammatically) the over-all structure by cross-cutting it in various places-in short, he attempts to make explicit the total structure, to make accessible to the observer all of the functioning parts and their mutual dependencies. 
e. When this has been achieved the linguist's task is completedhe has described a functioning structure.

II. What are some of the general characteristics of this procedure? a. It takes off from easily accessible constants-in this instance physiological; nature has, in great part, bounded the linguist's object of study for him: the first lines can, for the most part, be drawn by the gross anatomist.

b. It is explicit: the precise characteristics of the preliminary frame (within which the linguist segments and describes the continuum which is the linguistic process) can and must be stated; the precise criteria in accordance with which the linguist operates on the raw data yielded by the preliminary frame, the operations he performs on the raw data, and the order of such operations, can and must be stated.

c. It is repeatable: two or more linguists may take off from the same constants, apply the same preliminary frame, perform the same operations in the same order and in accordance with the same criteria, and-within the limits of flexibility of constants, frame, operations, and criteria-come up with the same elicited structure.

d. It is public: the entire procedure on all levels is open-or can readily be made so - to inspection by interested parties - the steps of the analyst can be retraced and his raw data can be rechecked. To these characteristics of the work of the linguist we owe the label "linguistic science."

III. But does the linguist feel that the job is done? In the course of his investigation, he has discovered variations in make-up and ordering of units which he fails to tie-in with similar variations in other elicited units. He is vaguely disturbed by these unelucidated variations, but has no means of coping with them within the arbitrary limits of his object of study, i.e. within the medium as he originally defined it. He tends to label these variations "free", but is unhappy about the label. In an interdependent universe, it seems to him unlikely that any variation is ever really "free". But science is long and life is short-an arbitrary line is drawn, he smothers his natural curiosity, and turns back to replow familiar territory. 


\section{B}

I. How does the student of culture go about finding the answer to his question?

It is much more difficult to portray, in any simple fashion, the procedures of the student of culture-particularly so for one who has come into cultural anthropology via the back door, and makes no pretense of operating as anything other than an interested-and we hope not too benighted-layman in that field. I present one such lay view; I make no apologies for the negative phrasing of these remarks; this is, for better or for worse, a linguist's-eye view of the activities of the culture analyst.

a. His attempts to delimit the object of study take not one but three different directions:

(1) He reasons (i.e. he talks) about the object of study and attempts to delimit it verbally; or

(2) he looks for manifestations of the object of study in act and in artifact, that is, for those manifestations which are immediately and directly accessible to observation, and proceeds to describe them in biological (or physiological) and physical terms; or

(3) he plies his informant with questions and records the answers to them verbatim, or, far more frequently, in an abbreviated form in which they have already been passed through the filter of the analyst's own interpretive frame.

b. He attempts to convert the raw data resulting from his reasoning, from his direct observation, or from his interrogation into a meaningful whole by further reasoning (i.e. talking) about it.

c. He attempts to make rational this whole by trying to equate its parts to items in a logical (i.e. verbal) system which serves as an explanatory frame.

d. He states the results of his analysis in a way calculated to bring out in the listener or reader a sense of a satisfying whole and a conviction that the particular unit under consideration fits into a larger meaningful context.

II. What are some of the general characteristics of this procedure? a. It takes off not from a single, but from several sets of con- 
stants-some of them biological, others physical, and still others verbal, i.e. the medium for the culture analyst is heterogeneous.

b. It is only in part explicit: there is not one, but a variety of preliminary frames (within which the culture analyst attempts to segment and describe the culture process); their precise characteristics can rarely be listed; there is no consistent distinction drawn between raw data as yielded by a preliminary frame and the finished results of culture analysis; the precise criteria for analysis are rarely stated and the operations performed and their ordering are rarely specified.

c. It is not repeatable: two or more culture analysts seldom take off from the same constants, rarely apply the same preliminary frames, infrequently specify operations or criteria, and seldom come up with the same elicited structure.

d. It is not public: seldom are the various analytic stages open to inspection by interested parties-it is almost impossible to retrace the steps of the analyst and his raw data are rarely susceptible to recheck.

III. Nonetheless, the analyst of culture may very well experience a feeling of satisfaction of a whole perceived, and may, very possibly, by his skill in presenting his thesis, persuade others of its truth. Furthermore, a sense of integration with a larger meaningful context may be derived from a perusal of such presentations. On the other hand, through lack of skill in presenting the results of his analysis, no matter what its quality, he may fail to persuade others of the correctness of his thesis. In such a situation he has no recourse but to start over again and make another try. No means for impersonal check are available to him. There is no court of appeal. He might at this point envy the linguist his ability to retrace (publicly) his steps.

C

My topic is "Cultural Implications of Linguistic Science". Hence a spelling-out of certain of these implications for the non-linguist may here be requisite:

(1) He should define the medium for a particular culture process.

(2) He should create, adequate to that medium, a preliminary frame for describing it. 
(3) He should list the criteria according to which the analysis is to be carried out.

(4) He should specify the operations to be performed and their ordering.

(5) He should state the results in a variety of ways.

There is good reason to believe that the definition yielded by following through on the first implication, for most aspects of culture, will turn out to be "language". If this is in fact so, then it is the linguist who will provide the culture analyst with the preliminary frame (language with its built-in segmentation) upon which the culture analyst, if he is to experience the satisfaction of a task completed, must perform his specified operations in their stated order in accordance with his listed criteria in order to elicit the structure of the cultural behavior reflected in this medium.

Be that as it may, a word of advice to the linguist, too, may here be in order: his procedures may justify the label "scientific," but so long as he works only within his arbitrary line, he will continue to frustrate his natural curiosity. He should permit himself, now and then, the luxury of an excursion into neighboring fields, not merely for the pleasure of plowing new territory, but also for the satisfaction of searching for answers to the question 'why?' 


\title{
III. The Spectrographic Analysis of Speech
}

\author{
Acoustical Vowel Relationships
}

GORDON E. PETERSON

\section{University of Michigan}

There is some strange fascination which the study of oral language holds. Wherever men have turned to learning, as occult, charlatan, or scientist they have studied language in some form. Within the University of today men who study oral communication come from fields as diverse as English, Psychology, Physics, Linguistics, Speech, and Electrical Engineering. Some of these individuals, we believe, work more to the point than others, but all of these fields are represented by particular interests in oral communication, individual methods of approach, and special objectives.

When we consider seriously the nature of the communication process, the fascination is not so strange. For linguistic communication is the highest order and most complex process in which human beings engage. In effective communication one must not only think, but in some manner the thoughts must be transmitted to other individuals.

In Figure 1 is shown a highly speculative schematic of the communication process which emphasizes a single individual. The signal is normally generated in the higher functional centers of the nervous system, suggested at the left of the diagram.

At the level of semiosis and semantics a symbolism must be formulated for communication. This symbolism we believe to be quantized or discrete in nature. The quantization probably has essentially the same form as other specific instructions to our neuro-muscular systems: "Touch the table." "Get that book." "Mail thisletter." Clearly, in these initial stages the symbolic formulation has the form of neural impulses (and possibly electrical fields). It is here that any quantizations or discrete units are to be found (if at all), for by the time the directives have set the motor neuro-physiology into operation, a relatively continuous flow of muscle movements and coordinated articulations are observed. 
The intermediate mechanisms which are indicated in the diagram are to perform certain of the primary functions which must be involved in such a system of signal generation. The Short Time Storage provides a systematic flow of instructions to the Controller of the Motor Speech Mechanism. The Comparator performs the feed-back function, which is now widely recognized as fundamental in many types of physical systems and as a common control mechanism in physiological organisms. The Comparator receives sensory signals which it compares with the instructions from higher neural centers. In the well functioning feed-back control or servo system the Comparator supplies a signal which just corrects for the failures of the output of the primary device to follow the original instructions.

The complex muscular actions and breath stream dynamics create a complex acoustical wave whose ever-changing form presents many problems in analysis. It is a matter of some importance that the acoustical speech signal does not completely represent the positions and movements of the speech mechanism. It cannot, for at times part of the mechanism is silent or in silent movement during the course of an utterance. There is little reason to anticipate, for example, that every detail of the motor production can be found in the acoustical speech wave: the precise movements of the palate or the size and shape of the tonsils. In fact, certain basic difficulties are to be anticipated in attempting to distinguish in detail those features of the acoustical speech wave which are produced by the laryngeal or other generating sources from those features which are due to the cavity resonances and shapings which lie above the particular source.

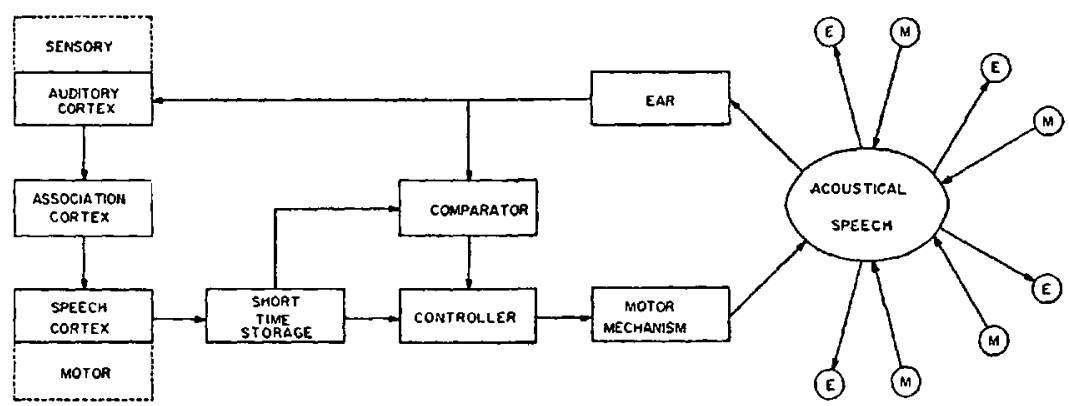

FIG. 1. Speculative model of the major individual functions involved in the communication process. 
It is primarily the acoustical waves of speech which are common to various observers. It is these that we may observe, compare, and preserve. Since individual vocal mechanisms differ appreciably, it is highly probable that individual speakers attempt to do whatever is required with their vocal apparatus to form acoustical waves which will provide a reasonably adequate communication. When the speaker's vocal or sensory mechanisms are sufficiently abnormal as to make such adjustments impossible, then some type of communication breakdown results which can sometimes be aided by prothesis and specialized training.

Highly complex, also, is the acoustico-neural transformation performed by the receiving ear of the listener. For the ear is nothing more than such a transforming device. It receives acoustical pressure waves at the input and into the cochlear branch of the auditory nerve are emitted neural impulses which conform in some complex manner to impinging acoustical signals. It must be recognized that ears differ markedly as well as vocal mechanisms. It should be noted that in the cochlear transformation of the receptive process, the ear can add nothing but noise to the acoustical signal in its operation. The auditory apparatus is a complex mechanism, capable of extracting useful information from acoustical signals under a multitude of environmental conditions. A knowledge of its function may aid in determining which aspects of the acoustical signal are significant in speech perception. Such knowledge would assist the experimentalist in determining what aspects, if any, of the signal might be discarded or disregarded in analysis, and what features are of primary importance. However, all of the auditory information which is supplied to the brain in an utterance is in the acoustical wave. Whatever the acoustical parameters of speech may be which are of primary importance to the human ear in its wave form analysis, they must be transformations of acoustical variables or of combinations of variables within the acoustical wave. Also, students of phonemics know exceedingly well, that in perception a listener does not always attend to just the same features of the acoustical speech signal.

Beyond the inner ear, however, once the signal has passed into the maze of neural fibers wherein it is perceived, then the forms which the signal may take in actual interpretation are probably varied beyond cataloguing. For the brain brings to the interpretation of the speech 
signal great quantities of remembered or stored information. The signal is perceived and interpreted in terms of both the past experience and the present physical status of the organism.

It is a matter of considerable importance, however, that from the time the symbolic formulation is initiated until the signal has passed through the cochlea of the ear, no further information intended by the speaker is introduced into the signal. As a matter of fact, information is very probably lost through every process involved in the signal transmission. Relatively few individuals would claim to be able to produce consistently, speech patterns which adequately represent the ideas they wish to transmit. It is commonplace to learn that our voices are not as charming nor as articulate as we command them to be. Masking noise and disturbing room reverberations commonly combine with the signal in its acoustical form, and the distortions of the human ear are well known.

While something is normally lost in each subsequent operation involved in the oral communication process, the speaker has a considerable amount of information fed back to him as a measure of the effectiveness of his communication. There is a kinaesthetic and proprioceptive sense of the movements of the speech mechanism which aid in its control. Likewise there is a direct feedback from the mouth to the ear, so that an individual hears his own speech productions and thereby is guided in their control. Serving a still higher order function is the feedback by speech and other means from the listener. Within certain limits, a speaker may judge the success of his communication from the responses of the target of the communication.

There is considerable interest in developing an understanding of the total neuro-physiological-acoustical process involved in linguistic communication. As indicated above, one of the primary states of the speech signal is its acoustical form. The remaining portion of this paper is devoted to the presentation of various acoustical measurements which have been carried out on relatively simple speech samples, largely obtained under controlled experimental conditions.

Probably the most prominent characteristic of the sound spectrogram is the appearance of the heavy dark bands of the speech resonances. These correspond to the formant frequencies. of the vowel portions of syllables. In Figure 2 is shown a set of measurements of the resonant frequencies on a series of simple vowels produced by a single 
speaker. The vowels were spoken in identical pairs directly into a sound spectrograph. The dotted lines divide the data into the two successive days on which the analyses were made. For the purpose of measurement, broad band spectrograms and amplitude sections were made of the recordings. $F_{0}$ represents the fundamental pitch of the voice, and the higher points are for the first three formant frequencies of the vowels. No recorded reference was available to the speaker.

In Figure 3 are shown various patterns for the frequency of the second formant plotted against the frequency of the first formant for a single speaker. In this figure the origin is shown at the upper right, which makes the vowel relationships somewhat easier to interpret for those accustomed to working in linguistics. In the monograph prepared by Professor Joos on Acoustic Phonetics, logarithmic scales were also employed. It is sometimes more convenient to employ a linear
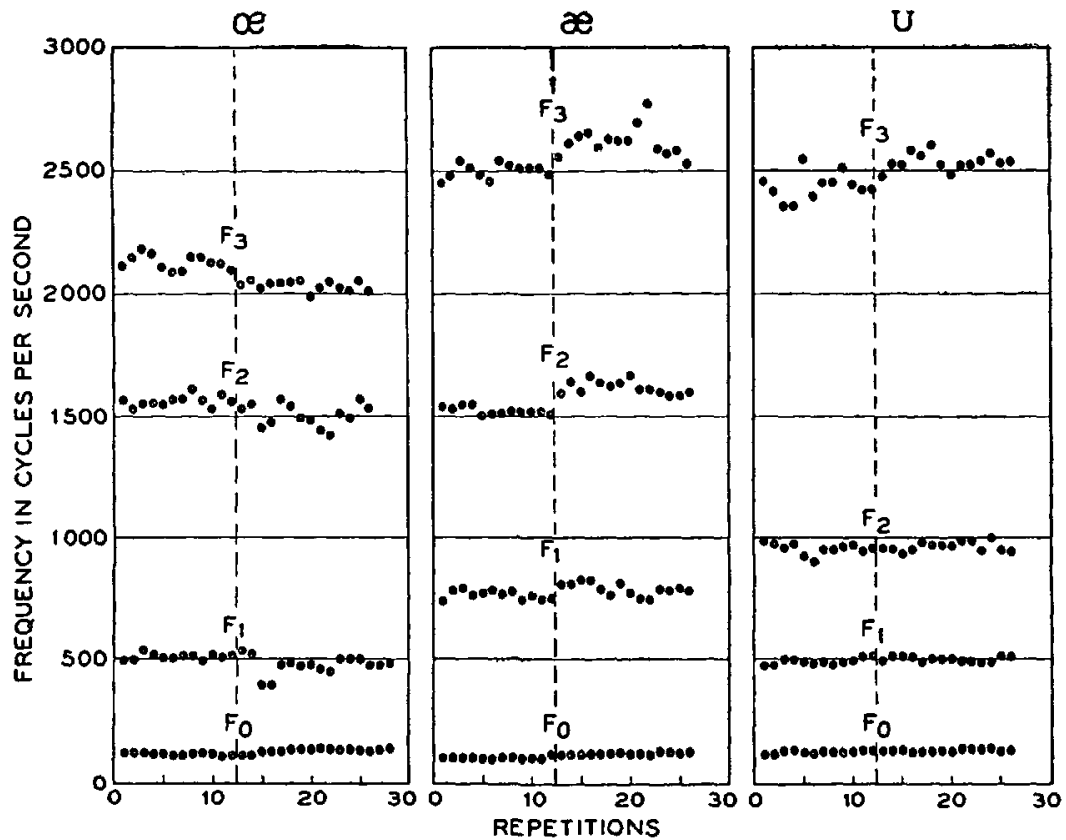

Fig. 2. Measurements of the fundamental voice frequency and of the frequency of the first three formants for three different vowels spoken by a single speaker on two consecutive days. 


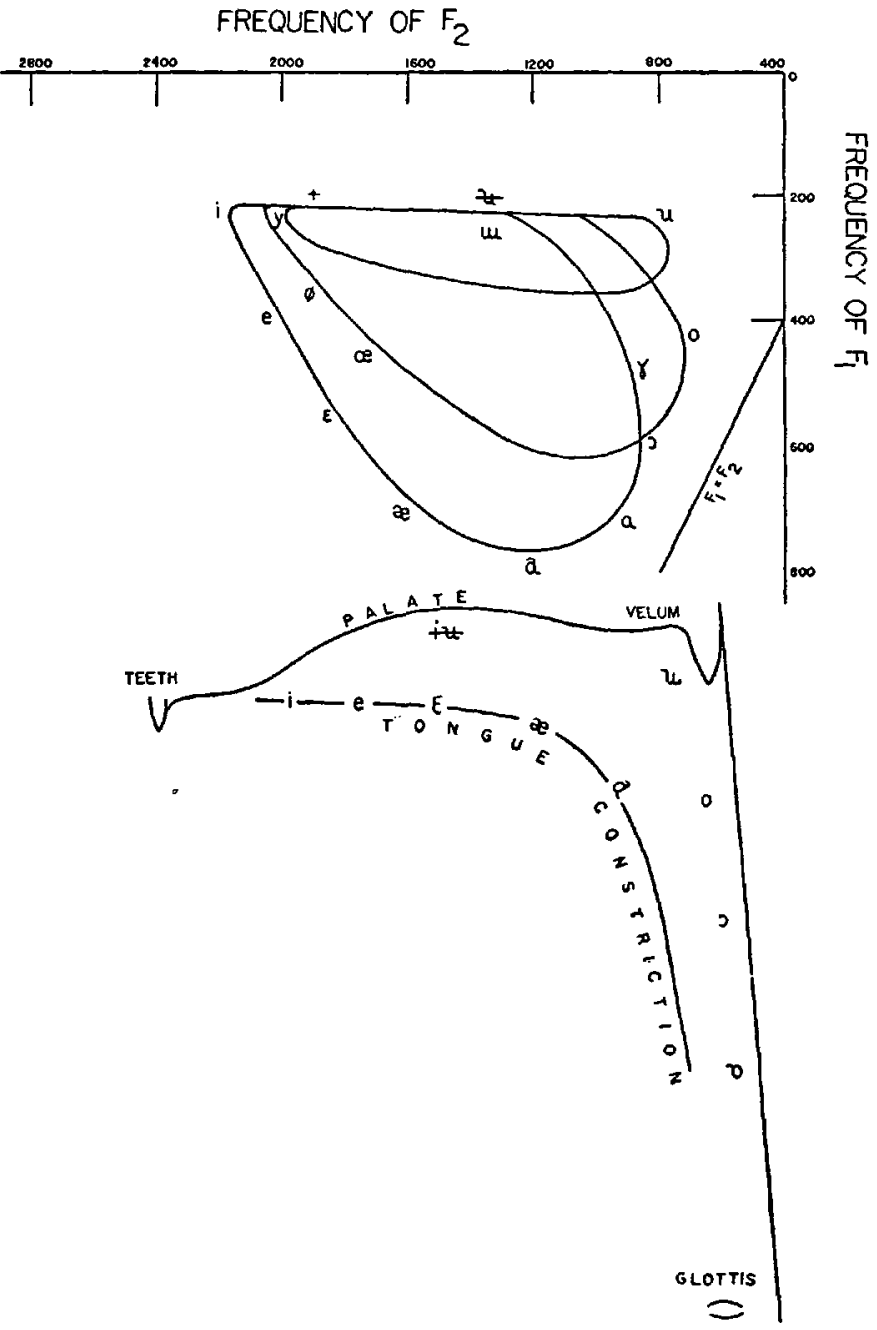

Fic. 3. The frequency of the first formant versus the frequency of the second formant for vowels produced by a single speaker. The three loops in the upper pattern were obtained with different degrees of lip rounding superimposed upon approximately identical tongue positions. The lower pattern is a schematic representation of tongue constrictions obtained from the examination of published x-rays of the rocal tract. 
scale for arithmetical or engineering purposes, but most of the experimental information which has been accumulated by the present author strongly favors the use of the logarithmic scale for phonetic purposes.

All three loops in the acoustical diagram represent corresponding tongue and jaw positions, with only lip rounding varied. In a schematic and simplified form, representative positions of maximum tongue constriction along the vocal tract are shown in the lower portion of Figure 3. Since the lip rounding is largely independent of the tongue position, only one set of vowels is shown, front unrounded and back rounded. It is obvious that the acoustical chart and the physiological chart do not have a simple correspondence.

In Figure 4 is shown an arrangement of equipment similar to that which has been employed to obtain equivalent speech samples for study. Reference utterances are recorded on the Magnetic Tape Repeater, and are reproduced over the loud speaker in a sound treated room. The subject is asked to listen to a reference utterance and to imitate some aspect of its phonetic characteristics, such as the phonetic vowel value, or the fundamental voice frequency, or the personal voice quality of the original speaker. The matched sample is recorded

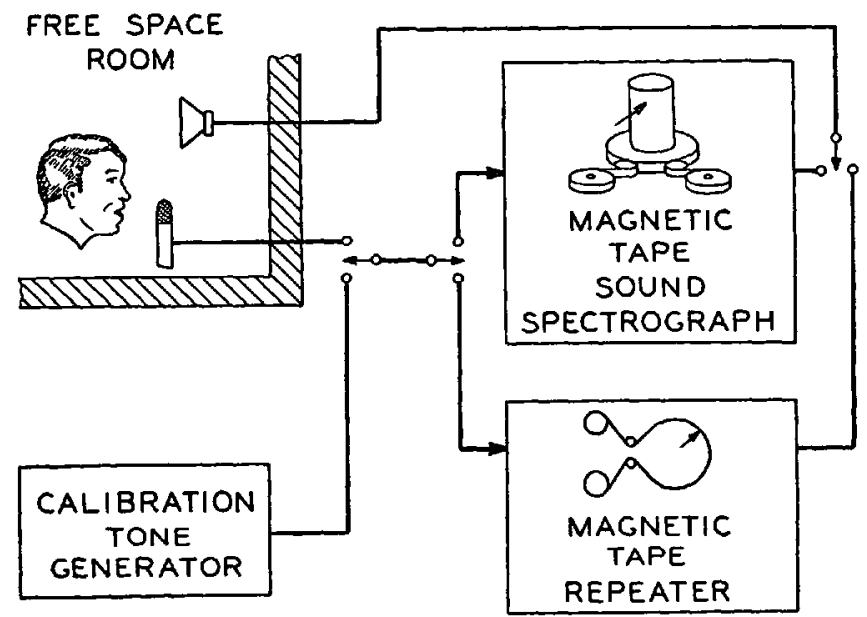

Fra. 4. Arrangement of equipment for obtaining matched speech samples for spectrographic analysis. 
directly on the Sound Spectrograph and can then be repeated through the loudspeaker for comparison with the reference. When a satisfactory match is obtained an analysis is carried out. The usefulness of a magnetic tape sound spectrograph for signal study and storage in such experimentation is relatively obvious.

Figure 5 shows data obtained on two vowels by the above technique. In this graph the frequency of the second formant is plotted against the frequency of the first formant. The points are for men, women and children subjects. The points shown with $x$ are for matches by the speaker who produced the reference sample. The straight lines pass through the origin so that they represent lines of equal formant ratio.

It has been observed previously that the positions and movements of the formant frequencies are of importance not only in the definition of vowel values, but also in the definition of the consonants. Figure 6

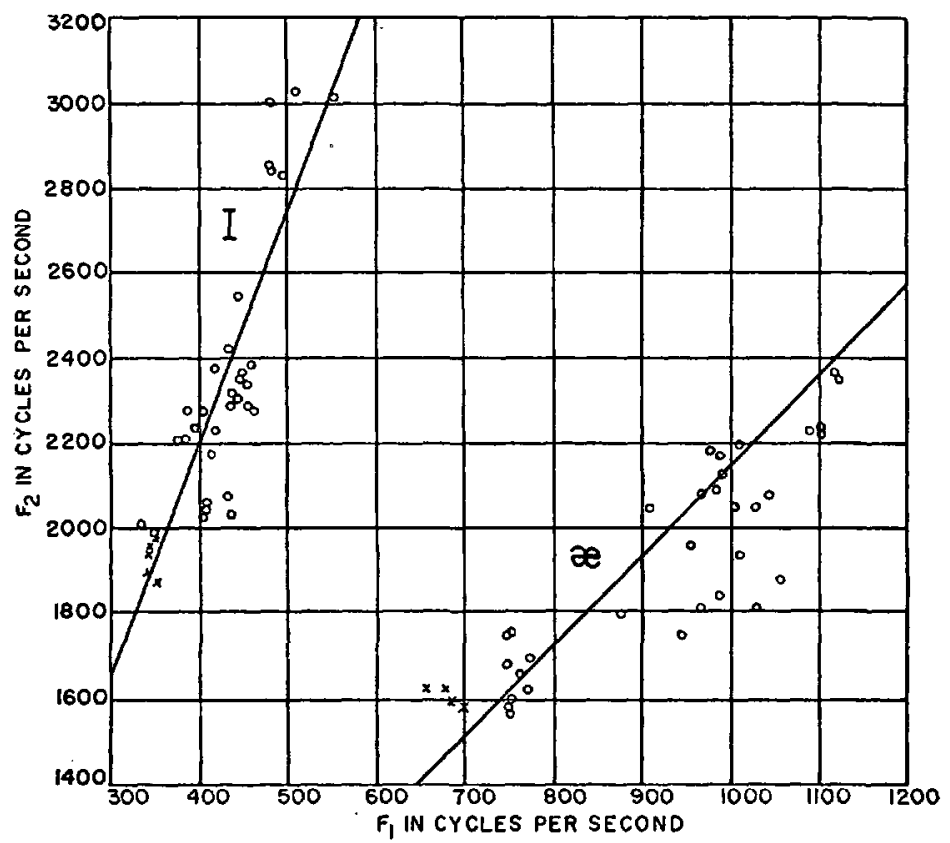

Fia. 5. The frequency of the second formant plotted against the frequency of the first formant for a series of vowels by men, women and children, matched to a reference by an adult male speaker. 


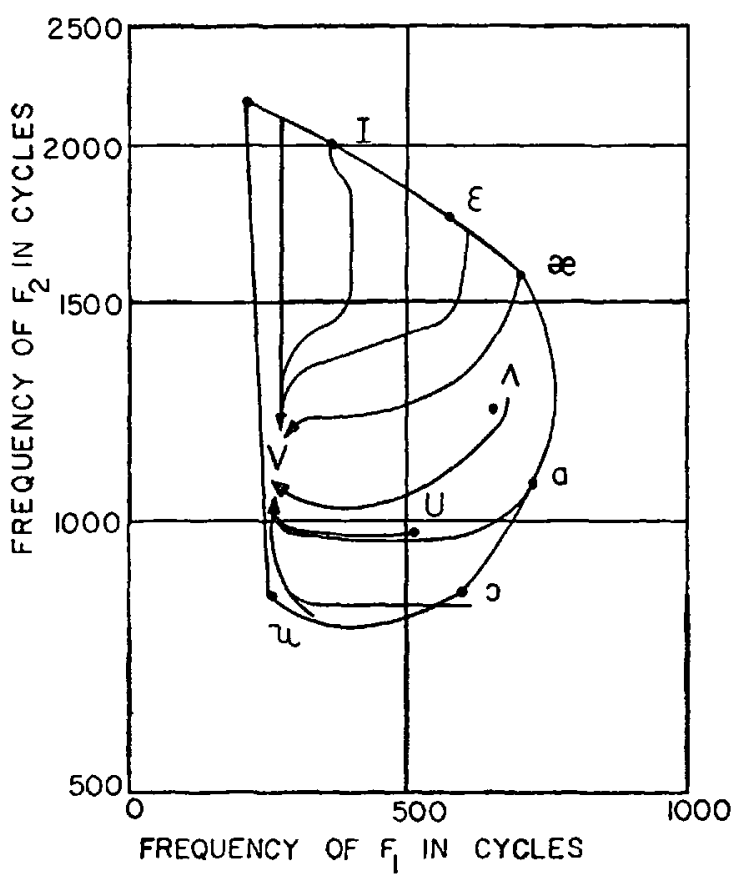

Fic. 6. Formant traces for vowels followed by a precise articulation of the consonant $/ \mathrm{v} /$.

shows traces of the frequency of the second formant versus the frequency of the first formant for utterances involving the various vowels plus the consonant $/ v /$. The points on the vowel loop are for the sustained vowel values by a single speaker. Each vowel-consonant utterance involves a movement to a relatively open identical consonant value. This pattern, of course, represents a very idealized series of utterances in which articulatory inter-effects have largelv been eliminated.

A much less idealized series of utterances is represented in various forms in Figures 7, 8, and 9. In Figure 7 are shown broad band spectrograms for the various vowels produced by a General American speaker followed by the consonant /f/. On a logarithmic plot, with the origin at the upper right, traces of the first two formant frequencies are shown in Figure 8. The arrow heads show the direction in 
which time progresses. Measurements of the formant values in constructing these plots were traced through into the frictional portion of the utterance as far as voicing and formant pattern could be observed. In Figure 9 are plotted measurements of the frequency values of the first three formants. The front vowels are shown in the graph at the left. The vowel portions of the utterances are equated in time according to the point of last formant measurement preceding the friction of the $/ f /$.

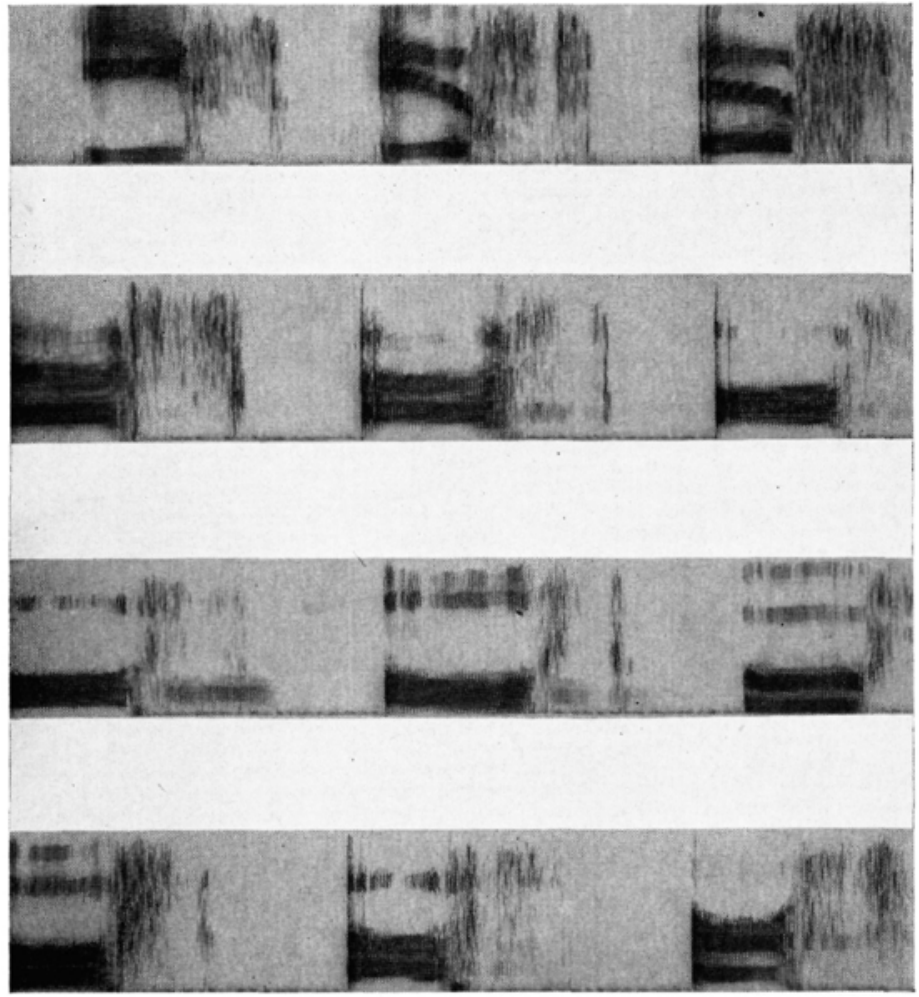

Fig. 7. Broad band spectrograms of vowels plus the consonant /f./

A.

B.

C.

D. if

æf

of

uf
If

af

of

Af ef

af

uf

3f 
FREQUENCY OF $F_{2}$

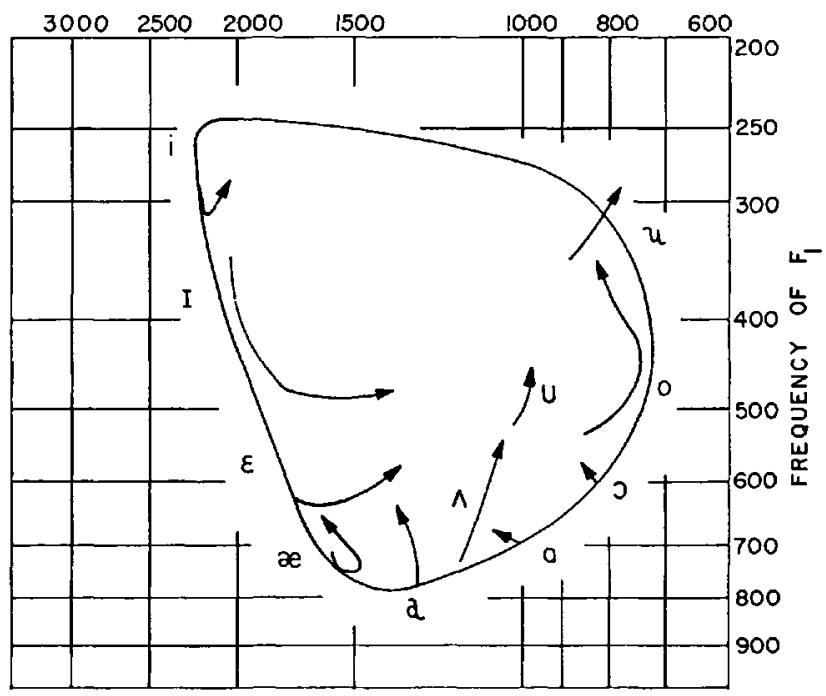

Fig. 8. The frequency of the first formant plotted against the frequency of the second formant for the spectrograms of Figure 7.

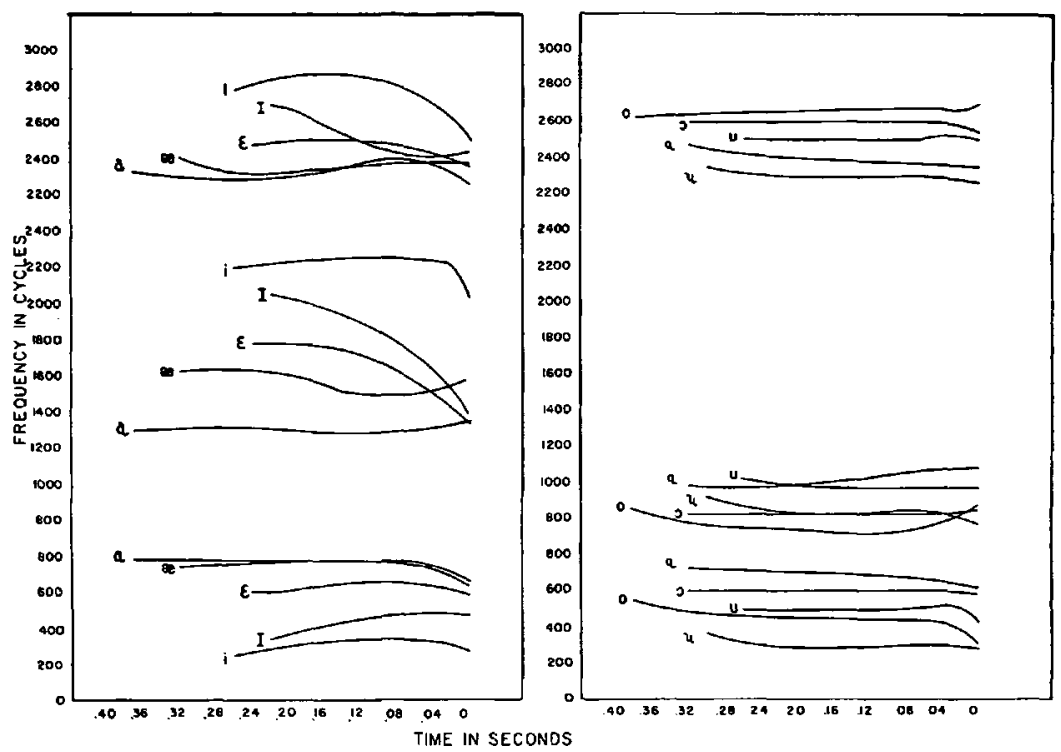

FIG. 9. Plots of the frequencies of the first three formants for the spectrograms of Figure 7 . The patterns have been equated in time at the termination of phonation. 
In analyzing the traces from this actual series of utterances, there does not appear to be any central point for any one of the three formants; nor is there an indication of such a point from the movements, singly or in combination of these formants. Since the movement from the vowel to the consonant and the formation of the consonant are effected by the preceding and following vowels, these results appear entirely reasonable. They suggest that the acoustical contribution of the formant movements to consonant identification can only be interpreted in terms of the phonetic environment of the consonant, so that a simple system of acoustical patterns for consonants is hardly to be expected. It should be noted that in actual speech the situation will likely be somewhat more complex than that represented in Figure 7 which is for a relatively isolated series of utterances. There is a very considerable need for experimental study with actual speech samples to determine the degree to which and the manner in which the vowel terminal and vowel initiating formant values contribute to phonetic recognition of the consonants. There is an even greater need of information about the frequency and time structure of the actual consonant portions of speech.

\section{Why and How Do We Study the Sounds of Speech?*}

\section{MORRIS HALLE}

\section{Research Laboratory of Electronics, Massachusetts Institute of Technology}

Many linguists and phoneticians view phonetics and phonemics as two completely separate disciplines. Phoneticians will sometimes claim that phonemic analysis is of no interest for phonetics, while phonemicists will say that their task begins where that of the phonetician ends. Phonetics, it is said, deals with speech as a physical and physiological phenomenon without regard for its social function and should, therefore, be classed among the natural sciences, while phonemics takes account of physical phenomena only insofar as these perform a definite function in language and is, therefore, a social

* This work was supported in part by the Signal Corps; the Office of Scientific Research, Air Research and Development Command; and the Office of Naval Research. 
science. ${ }^{1}$ Some linguists have gone even farther and have adopted as their slogan de Saussure's remark that in its essence the linguistic sign is incorporeal. ${ }^{2}$ These linguists do not admit appeal to physical facts of speech as arguments valid in lingusitic descriptions.

It is my purpose to show that a sharp distinction between phonetics and phonemics cannot usefully be maintained; that phonetics actually makes use of considerations which are strictly phonemic; that physical criteria are an integral part of phonemics; and that a description of language on any level, from phonetics to stylistics, cannot be properly evaluated without considering its consequences on all other levels.

We begin by examining the working procedures of phoneticians. All phoneticians utilize a notation consisting of discrete symbols, an alphabet; e.g., the IPA alphabet. This alphabet is central to the description to such an extent that one might say that a primary purpose of all phonetic descriptions is to give acoustical and/or articulatory meaning to the alphabetic symbols employed. No book written by a phonetician remains completely within the physiological or acoustical domain. No phonetician is ever satisfied just to classify the various articulatory movements or the great variety of wave shapes observed in speech without interpreting them in terms of an alphabet.

It is incorrect to say that a non-alphabetizing description makes no sense or lacks all scientific interest. We possess such descriptions of speech, and many among us have profited from them. We may mention here the work of the Bell System on the spectral distribution of energy in long time speech samples, ${ }^{3}$ Licklider's work on the intelligibility of distorted speech, ${ }^{4}$ Dudley's work on the Vocoder ${ }^{5}$ and many others. It is significant that none of these authors are phoneticians.

${ }_{1}^{1}$ This is essentially the view expressed in the introductory chapter of Trubetzkoy's Grundzuge der Phonologie, Travaux du Cercle Linguistique de Prague, VII.

2 Cours de linguistique générale (Paris, 1949) p. 164, also pp. 21 and 30.

'H. K. Dunn and S. D. White "Statistical Measurements on Conversational Speech," Journal of the Acoustical Society of America, XI (1940), 278-288.

${ }^{4}$ J.C. R. Licklider "The manner in which and the extent to which speech ean be distorted and still remain intelligible," Heinz von Foerster, editor, Cybernetics, Transactions of the Seventh Conference (Josiah Macy, Jr. Foundation, New York, 1950), pp. 58-122.

'H. Dudley "Remaking Speech" Journal of the Acoustical Society of America, $X I,(1939), 169-177$. 
Secondly, a phonetic description usually omits the individual voice quality. (I am not speaking here of the differences in voice quality that have emotional meaning, such as anger, doubt, affection, but rather of those that are specific to each speaker.) This is a very fundamental omission, and the reasons for it are quite obvious once we take into consideration the social function of speech. It cannot be justified, however, as long as we consider speech from a strictly physical point of view. ${ }^{6}$

We may say, then, that phonetics represents speech as consisting of temporally discrete events whose total number is quite limited. (No phonetic alphabet contains even 1000 symbols.) It also chooses to disregard the individual voice quality when making its description. In order to do this the phonetician must have some prior notions about speech; at the very least he must have confidence that his very special way of operating upon the phenomenon leads to interesting and reasonable results. This, however, can only be decided if we consider the function that speech serves, namely communication or the transmission of information.

Once this fact (the social function of speech) has been explicitly recognized, it is easy to justify the omission of the individual voice quality on the grounds that since this is a phenomenon over which the individual has no control, (he usually cannot choose between speaking in his own voice and that of another man) it cannot serve to transmit information.

The use of an alphabet, i.e. of a representation of the physical phenomena by means of discrete symbols assigned to intervals of varied duration, can also be justified only if the social function of speech is considered. As a medium for the transmission of information speech can be represented in this fashion. If, however, a faithful reproduction of the original utterance (and not just of the information content) is desired, as for example in a high fidelity recording, it would not be appropriate to represent speech in this fashion. And it is precisely because high fidelity criteria play an important part in satisfactory telephone communication (people want to sound natural and to hear their interlocutor's natural voice) that telephone engineers

- A very suggestive discussion of this question from the point of view of an engineer can be found in H. Dudley's "The Carrier Nature of Speech," Bell System Technical Journal, XIX, (1940), 495-515. 
have been less enthusiastic about linguistics and phonetics than many of us have wished them to be.

The number of properties reflected in a phonetic alphabet can be justified again only from a consideration of the function which language serves. The phonetician employing some system of transcription believes that it enables him to record everything of importance; which usually means everything that in his judgment may play a role in the transmission of information between speakers.

The social considerations should, however, not obscure the fact that all phonetic transcription systems, without exception, indicate certain properties of the speech wave. Thus, in striking contrast to the wide disagreements between phoneticians of various schools and backgrounds on how to record a certain vowel quality, there is almost complete unanimity on the number and the order of the symbols needed to transcribe a word like "bill." In general, all systems of phonetic notation indicate boundaries between vowels and consonants, between fricatives and stops, between nasal and oral consonants; they also agree on the order of these units in the sequence.

In other words, certain properties of the speech event are evaluated in an identical manner by almost all phoneticians. Such agreement is not conceivable unless we admit that there exist physical properties (and I want to stress the word "physical") which are common to a wide variety of (if not to all) human languages. It is these physical properties which enable a man with training in phonetics to deal with new languages. Had there been no such properties, phonetics would have little general interest.

In sum, then, the methods of phonetics clearly indicate that for the phonetician speech is not a physical phenomenon pure and simple: it is a physical phenomenon which "performs a definite social function, namely, the transmission of information." Phonetics, therefore, cannot be classified with physical sciences like chemistry, embryology or thermodynamics where no statements about the social functions of the objects investigated need be made.

We now turn to an examination of the procedure followed in setting up a phonemic system for a language. The first point to be noted is that the phonemicist does not perform his operations on the actual speech event, but rather on an alphabetic transcription of this event, which usually contains also indications of word boundaries. Since phonemics begins with a highly sophisticated symbolic representation 
of the speech event, and not with a direct observation of the physical phenomenon, there have been some linguists who have argued that the physical phenomenon is of no interest for phonemics. I shall show that this disregard for the physical facts leads to an uninteresting and trivial solution of all phonemic problems.

The most important principle employed in phonemic analysis is the famous criterion of complementary distribution. In a somewhat simplified form it states that a phonemic transcription omits all facts which can be inferred logically. In the language of information theory we could say that the criterion of complementary distribution is a rule for eliminating redundancy. For example, in a phonetic transcription of a certain language a phonemicist observes two types of stops, aspirated and unaspirated. He notices that the aspirated stops occur only before accented vowels unless preceded by $/ \mathrm{s} /$, whereas the unaspirated stops never occur in this position. He concludes then that there is no need for two kinds of stop symbols, since the appearance of one or the other of the symbols can be inferred from the context (before accented vowel except after / $/ \mathrm{s}$ it is always the aspirated; in all remaining cases the unaspirated).

(It should be noted that in most phonemic analyses the omission of a symbol from the phonetic transcription is accounted for by giving a rule of distribution. The set of these rules, plus indications of the pronunciation value of the phonemic symbols employed are usually considered an integral part of every phonemic analysis.)

We shall now examine a number of instances where the applicability of the principle of complementary distribution has raised serious discussion.

In English the velar nasal [ $\mathrm{n}]$ and [h] are in complementary distribution. Are these two units to be transcribed by one and the same symbol? If not, what limitation must we place on the principle of complementary distribution?

Another example which has recently been quoted in the literature ${ }^{7}$ : English possesses the sequences /skr/, /spr/, and /str/, but lacks the sequence /sr/. Should we write /sr/ instead of one of the three sequences above, thereby shortening the transcription by one letter? If not, why not?

A third example is the well-known case of "glimpsed" /glmpst/.

' Cf. G. H. Fairbanks' review of H. Lunt A Grammar of the Macedonian Language, in Language, XXX (1954), 125. 
Since there is no other word in English which ends in /mpst/, the /gli/ can be inferred from the context. Should we, therefore, transcribe this word as $/ \mathrm{mpst} /$ ?

Most linguists would reject the proposed simplifications on the grounds that the principle of complementary distribution is applicable only to units which are phonetically similar. ${ }^{8}$ No matter how we explicate the term "phonetically similar" it requires that we appeal explicitly to physical reality: to articulatory positions, to acoustical phenomena. We, therefore, conclude that phonemics (at least according to a great many of its practitioners) appeals explicitly to physical reality, and hence cannot be concerned solely with the "incorporeal" aspects of language.

If phonemics were regarded as completely non-physical, it would be extremely difficult to justify any limitations upon the principle of complementary distribution, since all such limitations appeal in some way to physical reality. Let us now examine what a phonemic solution would look like in which the principle of complementary distribution is applied without any limitations.

A theorem of information theory states that given a set of messages of known frequency, it is possible to devise an optimal code; i.e., a code where the information per unit will be minimal and the number of units per message will also be as small as possible, on the average. Such a code will possess only two units (zero and one) and erch message (i.e., word, sentence or utterance) will be composed of zeros and ones, according to a certain formula which need not concern us here. ${ }^{r}$ A phonemic transcription would then consist of long strings of zeros and ones. Such a solution would probably make even the most radical proponent of linguistics as a non-physical science shudder and protest that the Morse code is not properly a part of linguistics. ${ }^{10}$

It remains for us to investigate why a transcription which would

8 The most satisfactory explication of what constitutes "phonetic similarity" known to me is the one given by Roman Jakobson; cf. Preliminaries to Speech Analysis, M.I.T. Acoustics Laboratory Technical Report 13 (May 1952).

-D. Huffman "A. Method for the Construction of Minimum Redundancy Codes" Proceedings of the Institute of Radio Engineers, 40: 9, (1952), 1098-1101.

${ }^{10}$ For a different dissent from the view that phonetic reality is of no interest for linguistics see R. H. Stetson Bases of Phonology (Oberlin, Ohio, 1945), pp. 25-36. 
so admirably satisfy the requirements of a communications engineer would seem so completely wild to a linguist. The reason for this is that no linguist would be content with producing only a very efficient coding system for a language. Every linguist is interested in contributing towards a description of a language in its entirety. Phonetics, phonemics, morphology and syntax are all interrelated in that they serve to characterize different aspects of the language. It follows from this that a satisfactory description of a language can only be judged in its totality: thus it must be possible to go from a phonemic to a morphological and syntactic description on the one hand, and to the actual sounds (i.e., phonetics) on the other. It is clear that a phonemic description which accepts /mpst/ for "glimpsed" will have a very complicated morphology, because it will have to have among its rules for the formation of the past tense a special rule dealing with verb "glimpse." It will also have to contain a special rule for going from the physical stimulus to the transcription and vice versa; i.e., a complicated spelling system. Furthermore, there will be complications in the description of phoneme sequences; for instance, words without vowels will have to be admitted into English. There is no need to go on, since the conclusion is obvious: the simplification on the phonemic level entails a tremendous complication on all other levels of description. It is evident that the complications would be greater still, if we were to adopt an even more radical procedure of eliminating redundancy without regard for physical facts (e.g., the binary code mentioned above).

In linguistic descriptions, therefore, it is never useful to isolate totally any level from all others. It is always necessary to consider the effects of any statement upon other sections of the grammar. A proposed simplification can only be judged by its effects on the total description of the language.

Students of language, no matter what their field of specialization, are interested in the question of how human beings communicate by means of language in general, and by means of a given language in particular. This primary interest governs the way in which we study physical reality: the facts of physics and physiology are investigated in order to assess their role in the process of communication between people. This primary interest also makes it impossible for us to discount physical considerations, since real languages are not minimal 
redundancy codes invented by scholars fascinated by the powers of algebra, but social institutions serving fundamental needs' of living people in a real world. Finally, this primary interest forces us to evaluate all our statements by their effects on the description of language in its entirety.

A science of phonetics that disregards the social function of language is as trivial and barren as a science of phonemics that refuses to take into consideration the material aspect of language, its sounds. Neither phonetics, nor phonemics can get along without the other: phoneticians must have an understanding of phonemics, phonemicists must understand the methods of phonetics.

\section{Discussion}

There was a discussion after Professor Halle's paper.

CARLETON HODGE (Foreign Service Institute): I was wondering whether from the study of phonetics alone, that is, from the physical acoustical standpoint, it would seem probable that a new type of analysis could be evolved, something which is non-phonemic. It seems to me that we are still in the tradition of ancient Egypt. They first gave us individual consonants and phonemes, and somebody added the vowels later, and we have never gotten away from it. Do we have a chance?

MORRIS HALLE: I do not know why you want to get away from it-it has worked fairly well. But to answer your question, the high fidelity type of recording and the various kinds of speech compression devices which are being constructed by communication engineers, are precisely that kind of phonetics which are independent of segmentation of discrete symbolization. That is by no means a nonsensical way of doing things. It is a very sensible way, only that is what the engineer does. It gives you no possibility to do anything outside of it. You start with sounds; you will end with sounds, and that's all. You will not be able to make any statements about morphology in terms of wave shapes because you have set the pattern in which you can go on. The only thing that you can hope is that you can get out vibrations in the end, and they will to some extent be like the vibrations which you have put in.

EINAR HAUGEN: I just wanted to say that it is a long time since I have heard as sound a statement about the difference between pho- 
netics and phonemics. On the whole, we can say that in phonetics we have many symbols and relatively few rules, while phonemics has fewer symbols and more rules. The argument is whether we shall create a complicated transcription and simplify our statement of phonetic rules or whether we shall have the other kind, and I think in most cases the answer is a practical compromise between the two opposite desires to have simple rules for pronunciation and simple transcriptions. It doesn't seem to me that anyone can speak ex cathedra and say "This is the phonemic system," in view of the fact that there are many phonemic systems depending upon where we choose to draw the line between the two.

PAUL GARVIN: I think that my colleague from MIT has put his finger on one of the basic controversies in analytical linguistics today, which is the famous question of levels. We all feel that from the operational point of view the various levels of analysis should be kept apart. All of us, I think, have enough Sprachgefühl for our own language and other languages to know that they are really not clearly separate in practice, and this is no more than a restatement of the general truth, I believe, that in the world everything is interrelated, and then conceptually you have to sort it out, and how much in the process of sorting out you can keep separate, and how much you can keep together, I think is one of our basic problems.

MORRIS HALLE: We do not all feel-and among all I am to be included-that the levels are to be kept apart. It is quite possible to make a very reasonable description of the sounds of a language in terms of wave shapes or motions of articulatory organs. It has never been done, but in principle it is certainly possible. How would you state, for example, the rules of the formation of the English past tense or the rules of the formation of plural in English in terms other than phonemes. The beautiful thing about the phoneme is that it provides for the possibility of stating very elegant and beautiful rules. If you use any other type of transcription, this goes out of the window. If you want to argue "therefore phonemes exist," because this gives you the nicest description, that is all right. We can go a long way by using the phonemes. This is in part my answer to Mr. Hodge. Really there is no need to look for other types of description. Linguists have always been interested in describing language on all levels, as far as I can see. This keeping levels artificially apart, I think, has never 
worked very successfully, because then you end with the type of analysis such as that of Hjelmslev. Hjelmslev suggested transcribing all voiceless consonants in Danish with an $h$ before the symbol for the corresponding voiced consonant because he wanted to show that he did not care about the physical reality. Why not use a binary code, which is much more efficient?

PAUL GARVIN : I was not quite referring to that. I was referring to the discussions of morphemic and phonemic segmentation and morphophonemics and so on, in the controversy about Pike's grammatical prerequisites. $I$ think they go back to this basic problem: to what extent can you treat phonemics and morphemics separately and to what extent can you not, and I do not think that we have as yet a definitive answer.

ROBERT STOCKWELL: (Foreign Service Institute): It seems to me that $\mathrm{Mr}$. Halle has tried to bring back together something which appears to be a dichotomy: phonetics on the one hand and phonemics on the other. Particularly, he seems to have been speaking about instrumental phonetics. At least, I will make that assumption since he is on the panel for spectrographic analysis. I would like to point out that phonetics itself has to be dichotomized into something we can call instrumental phonetics and something which has been called by George Trager and others microlinguistic phonetics. This is on the level of social science, not on the level of physical science. You write down in some kind of speech symbology the things which individuals with their human ears and without any instruments whatsoever are able to distinguish from each other as different sounds. This kind of phonetics has not been adequately handled or differentiated from instrumental phonetics today, and it is this differentiation which seems to me to be very important.

MORRIS HALLE: I am somewhat at a loss to make a comment on this. I think that one of the things with which I have been most impressed is that as I have learned more I have been able to distinguish more. Now I know that Mr. Smith is able to distinguish even more. Who is to be the standard for how many symbols you are to employ? Is it my ear, Mr. Smith's ear, or somebody else's ear? In such impressionistic phonetics one is so terribly dependent on one's own experience, that one can very well say the shortcoming of this method is that it is not uniform. And you will always have to write, "This transcription 
was made by me in such and such a year," and then you will give your whole biography as far as it is relevant. I don't see why we must distinguish. I would say this is a bad move.

ROBERT STOCKWELL: I fully agree that the best training a linguist can get for his ear is working with the sound spectrograph. I don't think there is better training that a linguist can get in phonetics anywhere than working and checking his recordings against what he can see by reading sound spectrograms. But the sound spectrogram itself, I believe, cannot contribute directly to the phonemic analysis that the linguist is trying to achieve in order to go ahead and do his morphological and syntactic work also.

\section{Time Measurements in Speech Analysis}

\section{T. D. HANLEY}

\section{Purdue University}

We have time on our hands this afternoon, time--one dimension of the multi-dimensional bio-acoustical time series that is speech. Now, let us agree at the outset that to mistake the single parameter for the complex phenomenon, the part for the whole, is to err in a naive, yet very serious way. Therefore, while my remarks will be concerned almost entirely with the single parameter, I shall not be unmindful of cause and effect relationships involving other parameters.

I have chosen to discuss the attribute of time because, among other reasons, it has been a somewhat neglected factor, one which I feel has been taken too much for granted by recent investigators. There has been a tendency to produce speech displays, including time as one dimension, and then to concentrate attention on other sound attributes. This procedure may lead to serious error, a conclusion which you may reach with me after hearing some of the research results to be presented to you this afternoon.

Despite the neglected status of the time attribute, there has been good research done with it. My paper today will be an attempt to show, by means of selected examples, the depth and breadth of time research. I feel, however, that before these research investigations are brought up for consideration, it will be profitable to examine some of 
the conventional methods for making time analyses. One of the simplest techniques is the use of the stop watch. Use of the stop watch permits the timing of selected samples of speech. By simple computation the rate of the speaker in words per minute is revealed. The reliability of the technique is dependent in large part upon the reaction time of the investigator and upon the size of the sample of speech analyzed.

An instrument which offers considerably more in the way of versatility and reliability is one (Fig. 1) originally designed and constructed at the Purdue Voice Communication Laboratory. This is

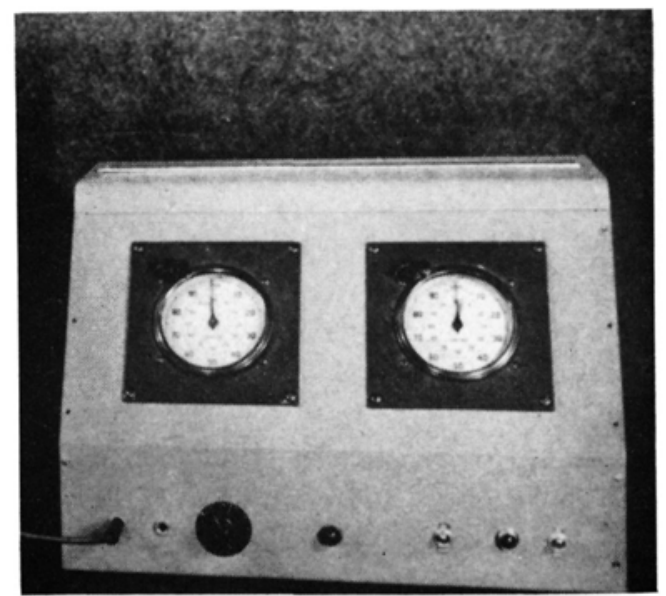

Fig. 1. Phonation Timer.

known as a Phonation Timer. It consists of two electrically operated clocks. When the operating switch is thrown, the clock on the right begins immediately to record time in conventional units. The clock on the left, however, is caused to operate only by means of a voiceactivated electronic relay. This means that the clock on the left will accumulate time units only during such time as voice signals of a predetermined magnitude activate the switching mechanism of the clock. By means of the right hand dial one may calculate words per minute just as may be done in the case of the stop watch. By means of the left hand dial, further calculations may be made, such as the phonation time ratio, with duration of voicing as the numerator in the fraction and total time of speech sample (taken from the right hand clock) 
as the denominator. Words per minute exclusive of pause time is a calculation which may be made from the left hand clock alone. This has been found to be an interesting and significant statistic.

There are three other common methods of doing time analysis, all making use of the records of instruments whose primary function is the measurement of some other acoustical attribute. From the power level recorder, for example, time measurements may be taken (Fig. 2a). The recording tape of the power level recorder passes the recording stylus at a known, constant rate. Each time the stylus of the instrument departs from the baseline, the existence of sound in time is recorded on the tape. Each time the stylus returns to the baseline, a silence period is recorded. It is possible, then, to "alculate such values

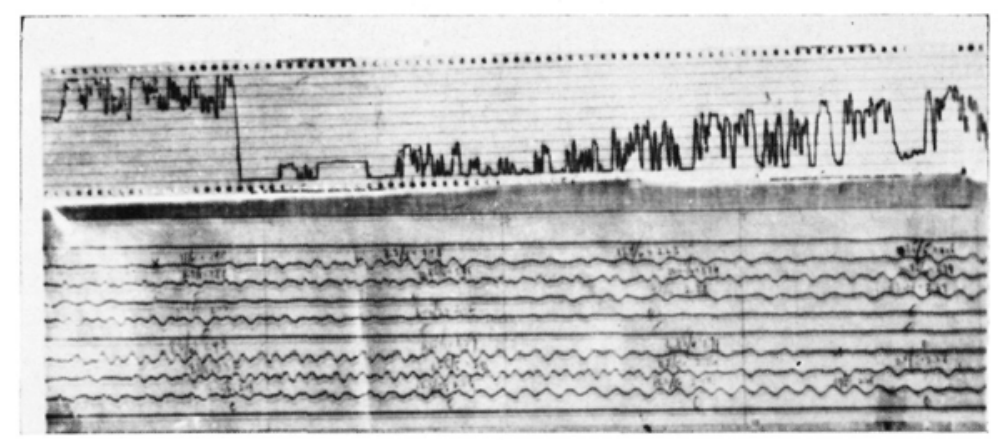

Fig. 2. a) (Top record) Trace from Power Level recorder. b) (Bottom record) Phonellogram, used in Vocal Frequency Measurement.

as phonation time ratio, words per minute, and even, if proper word or syllable identification can be made on the record, duration of inindividual speech units.

From pitch or vocal frequency records time analysis also may be done (Fig. 2b). Typically, in pitch analysis, speech waves from a microphone or phonograph pickup cause the oscillation of a tiny mirror which reflects a beam of light onto photo-sensitive paper passing by the lightbeam at a known rate of speed. Voice time is seen, of course, as the series of waves, pause time as the straight line portion of the record.

The third of these somewhat indirect methods of time analysis makes use of the Sonagraph or Visible Speech device. Here a sample of speech 2.4 seconds in duration is analyzed for the presence of energy 
at frequencies from zero to 8,000 cycles per second. Again, time is a constant value on the horizontal axis of the record produced by the instrument. Therefore, it is possible by visual identification of indi-

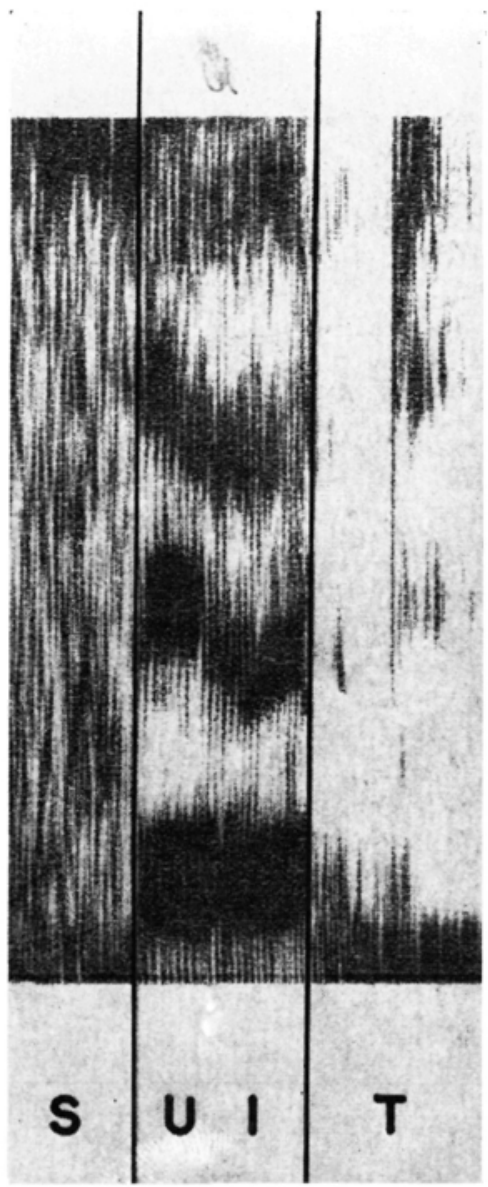

FIG. 3. Sonagram of Word "Suit" with vertical lines separating Vowel from Consonants for Duration Measurement.

vidual speech sounds to measure the absolute duration of each (Fig. 3). The list of time measurement techniques just presented does not exhaust the possible methods, but it does include those most commonly used. Several of the researches which I shall discuss this afternoon make use of one or more of these techniques. 
Four different frames of reference have been selected for discussion of the time attribute this afternoon. These are: the contribution of time to intelligibility, the contribution of time to judged excellence of speech, time as an attribute which modifies the perceptual aspects of other basic attributes of speech, and time's contribution to the perception of regional and foreign dialect and thus, by inference, its potential contribution in language training. For each of these four frames of reference, one or more researches have been selected which will point up the contribution of time. The investigations are not all those of the speaker; in fact more than half of them are not. Information will be presented, therefore, from some published materials which you may have read. My contribution, I hope, will be the integration of the investigations into a meaningful pattern.

\section{TIME AND INTELLIGIBILITY}

In the consideration of the contribution of time to intelligibility, an investigation by G. L. Draegert ${ }^{1}$ provides interesting data. Draegert's research design was an interesting and a fairly simple one. He administered a standard intelligibility test in noise to 250 subjects, young adult male college students at Purdue University. On the basis of the test results, Draegert selected for analysis the voices of 88 of his subjects, the two tails of his intelligibility distribution. That is, he analyzed the voices of the 44 most intelligible and the 44 least intelligible of his subjects. Some fifteen voice analyses were performed on the recorded intelligibility test lists of discrete words and on a short prose passage read by the subject. Of these analyses, two are of immediate interest to us this afternoon. One was syllable duration, the other phonation time ratio. Now it should be noted that his two groups, superior and inferior speakers in terms of intelligibility, were distinguished by mean scores on the criterion intelligibility test of $63.3 \%$ for the superior group, $33.3 \%$ for the inferior group. This difference was statistically significant at the $1 \%$ level of confidence. The two groups were found to be distinguished on the basis of mean syllable duration, by group mean scores of .174 second per syllable for the superior group compared with .141 second per syllable for the inferior group. This difference between groups was statistically significant at the $1 \%$ level. Finally the two groups were distinguished by the phonation time ratio, by values of .585 for superior group versus .504 for inferior group. This difference also was statistically 
significant at the $1 \%$ level. Draegert then performed a further statistical analysis, calculating coefficients of correlation between intelligibility and the several voice variables measured. The two variables in which we are interested-phonation time ratio and syllable duration-were found to be correlated with the criterion intelligibility score by coefficients of .35 and .92 , respectively. Draegert thus demonstrated that the contribution of time--here specifically syllable duration-to intelligibility is extremely important when the individual is attempting to communicate in an atmosphere of noise, where the masking effect of the ambient sound is considerable.

A second investigation in the area of intelligibility, in this case vowel recognition, has been reported by W. R. Tiffany ${ }^{5}$. Tiffany's ingenious research procedure included selecting tape recorded samples of the 12 commonly distinguished vowels of the General American dialect. He then cut vowel segments of $.08, .20, .50$ and eight seconds duration from each of the recorded vowels. These vowel segments were placed in random order on a master tape. They constituted the set of recorded stimuli for which responses were made by a group of trained listeners. The listeners responded to each sound stimulus by writing one of the given list of 12 phonetic symbols for each sound heard. Among the many significant results of the study were the following:

1. For the vowel $/ \varepsilon /$

a) at .08 second duration, $19 \%$ identification as [r]

b) at 8 second duration, $33 \%$ identification as [æ]

2. For the vowel $/ a /$

a) at .08 second duration, $64 \%$ identification as $[\Lambda]$

b) at 8 seconds duration, $9 \%$ identification as $[\Lambda]$

3. For the vowel /e/

a) at .08 second duration, $68 \%$ identification as [I]

b) at 8 seconds duration, $25 \%$ identification as [ $\varepsilon$ ]

It may thus be seen from Tiffany's work that vowel identification is at least in part a function of the duration of the vowel. Let me state here the obvious point that harmonic structure is the prime attribute contributing to vowel quality and hence to identification. But it is, I believe, noteworthy to find that the factor of time has so significant a contribution to make in the intelligibility, or the recog- 
nition at least, of the vowel sounds. With vowel duration shown to be so important to listener recognition, a question is raised as to the validity of any instantaneous display of vowel spectrum, at least insofar as any inferences regarding perceptual quality of the vowel are concerned.

It should be recalled that General American vowels, listened to by native speakers of the General American dialect, were the stimuli employed in the Tiffany study. It occurred to us that there might be profit in attempting to discover whether results similar to Tiffany's might be obtained with vowels from another language, listened to by native speakers of that other language. Accordingly a procedure very similar to Tiffany's was followed, using three Spanish vowels as phonated by a Colombian student at the University, listened to by a panel of seven Colombians. The vowels selected for study were /e/, $/ \mathrm{a} /$ and $/ \mathrm{u} /$. A tape was made up with the vowels presented with varying durations in random order. Since the listeners were not trained phoneticians, they were instructed to record their judgments on each stimulus sound in terms of key words. That is, the judgment sheet provided them was ruled into columns headed "e in peso," "a in dado," "u in pudo", etc. Following the definitions of the Spanish phonetician, Navarro Tomás4, judgment possibilities were set up for open and close [e], middle, velar and relaxed [a] and open and close [u]. Certain foil possibilities, such as the accented [i] in "ridículo" and the final vowel in "pudo" were included also. As is to be seen in Table 1, there appear to be, with Spanish vowels as well as General American vowels, recognition effects attributable to duration differences. The shortest of the /e/ stimuli, for example, was identified as either an open or close [e] $43 \%$ of the time but was also identified as an [a] $24 \%$ of the time and as [u] $10 \%$ and [i] $19 \%$. Duration thus contributed to discrimination between variants of the same phoneme and to erroneous identifications with other vowels. It is to be noted in this table that the errors were most numerous when the durations were most abnormal, that is, materially shorter or longer than the normal durations of the vowels. Many other comments about specific results displayed in the table might be made. However, it must be remembered that this was a pilot study in which the results were not subjected to tests for statistical significance. It was designed to discover whether duration of Spanish vowels might constitute a fruitful 
TABLE 1

Listener Identification of Spanish Vowels of Varting Durations Identification in Percent

\begin{tabular}{|c|c|c|c|c|c|c|c|c|c|c|}
\hline Vowel & Duration & 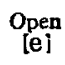 & $\underset{\text { lel }}{\text { Close }}$ & $\underset{\substack{\text { Middle } \\
\text { [a }}}{\text {. }}$ & $\begin{array}{l}\text { Velar } \\
\text { [a] }\end{array}$ & $\begin{array}{c}\text { Relaxed } \\
\text { [a] }\end{array}$ & Open & $\begin{array}{c}\text { Close } \\
\text { [u] }\end{array}$ & $\begin{array}{c}\text { Close } \\
\text { [i] }\end{array}$ & $\begin{array}{l}\text { Relaxed } \\
{[0]}\end{array}$ \\
\hline \multirow[t]{4}{*}{$/ e /$} & .060 & 14 & 29 & 14 & 10 & - & - & 10 & 19 & - \\
\hline & .087 & 24 & 48 & - & - & - & - & 10 & 10 & - \\
\hline & .113 & 29 & 48 & 14 & 一 & - & - & - & - & - \\
\hline & .500 & 29 & 21 & - & - & - & - & - & 29 & - \\
\hline \multirow[t]{6}{*}{$\mid a /$} & .060 & 10 & - & 43 & - & 14 & - & 10 & - & 10 \\
\hline & .087 & 10 & - & 29 & 29 & - & - & 10 & - & 10 \\
\hline & .113 & - & - & 33 & 24 & 14 & - & - & - & - \\
\hline & .133 & 10 & - & 48 & 29 & - & - & - & - & 10 \\
\hline & .140 & - & - & 50 & 9 & 14 & - & - & - & 14 \\
\hline & .500 & - & - & 21 & - & 29 & 14 & - & - & - \\
\hline \multirow[t]{5}{*}{$/ \mathrm{u} /$} & .060 & - & 24 & 14 & - & - & 19 & 24 & - & - \\
\hline & .073 & - & - & 19 & - & - & 10 & 57 & 10 & - \\
\hline & .087 & - & - & 10 & - & - & 19 & 52 & - & 10 \\
\hline & .100 & - & 24 & - & - & - & 19 & 29 & - & 14 \\
\hline & .500 & 29 & - & - & - & 一 & 14 & 21 & - & - \\
\hline
\end{tabular}

area for full scale, well-controlled research, which apparently it is. For the language professor, this information, if it is found to hold up in further investigations, may be quite significant in the teaching of vowel quality. A student who fails to achieve correct pronunciation by other techniques, may be aided by instruction to shorten or lengthen a given vowel sound in a given context. This is not to imply that such instructions are not occasionally or perhaps even frequently given. However, it is to imply that normative data for vowel durations in different contexts might be of considerable worth to the teacher of languages. Further, the results suggest that when the professor wishes to provide an auditory model for his student, he should do so in context and should not prolong the vowel.

TIME AND JUDGED EXCELLENCE

In the consideration of time factors as contributory to judged excellence of speech, I wish to go back now some 20 years to a study performed by Murray and Tiffin ${ }^{3}$ at the University of Iowa. In the 
investigation the general effectiveness of the speaking voice of each of approximately 1,000 freshmen was rated on a ten-point scale by members of the Department of Speech at the University. From those 1,000 freshmen, 171 subjects were chosen. Of these, the first or Poor group consisted of 88 of the poorest rated voices. The second or Good group consisted of 46 of the best rated voices. A third group, called the Trained group, was made up of 23 instructors and graduate or advanced students in the Speech Department. These 171 subjects were recorded speaking a 33 word sentence. From the recordings, several specific analyses of voice variables were made by the investigators. The results of all of the analyses are considered to be of significance to the speech teacher but three of them are most revealing with respect to our subject today. First, the average duration of individual phonations served to distinguish the Trained and Good groups from the Poor. On this variable both good and trained voices achieved an average of .13 second per phonation whereas the Poor group achieved an average of .11 second. A second factor which served to distinguish the Good group from the Poor group was variability of duration of phonations. The good voices showed a $19 \%$ greater average deviation in duration of phonations than did the poor voices. In the third factor, variability of pause time, the Good group was $28 \%$ greater in variability than the Poor and the Trained group was $26 \%$ greater than the Good, and $60 \%$ greater than the Poor. Thus duration of individual phonations, variability of duration of phonations and variability of duration of pauses served in this study to distinguish good and trained voices from poor voices, and further demonstration was made of the contribution which the time element makes to the total perception of speech.

\section{TIME AND THE PERCEPTION OF OTHER ATTRIBUTES}

Next, the contribution which time factors may make to the perception of other acoustical or perceptual attributes of the total speech phenomenon may be considered. The contribution of time factors to the perception of pitch has long been recognized, as indicated by the fact that most studies having to do with pitch and pitch variability include an analysis of the rate of pitch change per unit of time. The contribution of time to stress or emphasis has been thoroughly investigated in a study by Tiffin and Steer ${ }^{6}$. The 20 subjects used in 
this study were required to read a single sentence, "President Roosevelt wants all men to be loyal to the nation," five times. On each successive reading a different word within the sentence was designated to be stressed by the subjects. The recordings made of the performances of each speaker were subjected to various analyses, including pitch, intensity and duration. In the duration analysis, a comparison was made of the stressed and unstressed repetitions of the same word in each of the succeeding sentences. In this analysis it was found that $98 \%$ of the stressed words were of greater duration than the same words when unstressed. In summarizing their results, Tiffin and Steer stated their belief that of the six measures which they found to be significant, probably the most significant measure in the determination of emphasis was the greater duration of the stressed words.

TIME AND DIALECT

The final relationship to be considered in this paper is that between time and perceived dialect, whether regional or foreign. In this section I shall report the results of two separate investigations. The first, the doctoral dissertation of the speaker ${ }^{2}$, was concerned with the dialects spoken in the three major dialect regions of the United States, the Eastern American, the Southern American and the General American regions. In this study, college students in the three dialect regions were recorded reading a standard passage and speaking extemporaneously. The subjects were selected originally on the basis of length of residence within the dialect region and on the basis of judged "normality" of voice. The recordings made were further subjected to judgment by phoneticians at Louisiana State University and Columbia University where the "representativeness" of each dialect sample was the critical facor. By means of this judgmental procedure, an original population sample of 67 was reduced to 27 , nine from the General American region, eleven from the Southern American region and seven from the Eastern American region. The recorded speech samples of the speakers from these three regions were then subjected to instrumental analysis for pitch and duration factors. The duration measurements were made by means of the sonagraphic technique previously described. That is, sonagrams were made for the complete reading passage for each of the subjects in the study. Forty-nine individual sounds or sound combinations within the speech sample of 
each subject were then identified, marked and measured by the placement of a ruled templet over the sonagrams. The duration, in hundredths of a second, was noted for each individual sound. When the data obtained by this method were analyzed statistically, it was found that the durations of 13 of the 49 sounds were significantly different from dialect group to dialect group. These differences were in the direction of longest duration for the Southern American group for nine of the sounds and the Eastern American group for four of the sounds. The data collected are insufficient for broad general conclusions but they do suggest that sounds which are stressed tend to be relatively greater in duration for the Southern American group while unstressed sounds, perhaps benefiting from greater articulatory precision in the Eastern American speech, tend to be relatively greater in duration for that group. No sounds were found to be significantly greater in duration for the General American group.

It seemed desirable recently to determine whether the sound duration differences attributable to regional dialect might also be observed in foreign dialect. Accordingly a pilot study was conducted at Purdue during the last month. In this study five Latin Americans, Colombians, and five speakers of the General American dialect participated. Each subject read three English sentences which were recorded on high fidelity magnetic tape recording equipment. The sentences were so constructed that they provided two samples of each of the vowels $/ \mathrm{i} /, / \mathrm{e} /, / \mathrm{a} /, / \mathrm{o} /$, and $/ \mathrm{u} /$. In each case the vowel occurred between two plosive consonants, making for easy identification of the vowel sounds in the sonographic analysis which later was performed. By means of the templet technique described above the durations of the five vowels for the two groups of speakers were determined, and tests of statistical significance were applied to the obtained results. As is to be seen in Table 2, differences between groups significant at the $1 \%$ level were found for three sounds $/ i /, / \alpha /$ and $/ u /$. For all three, duration was greater for the General American group. A difference significant at the $5 \%$ level was found between groups for the sound /e/, but in this case the duration was greater for the Latin American group. There was no significant difference for the sound /o/. The obvious conclusion to be drawn from this study is that we should look for vowel duration differences in foreigners speaking our language. By logical inference, the obverse also is true: American at- 
TABLE 2

Vowel Doration Analysis of General American and Latin American Groups

\begin{tabular}{|c|c|c|c|c|c|}
\hline \multirow{2}{*}{ Vowe! } & \multicolumn{2}{|c|}{ Duration (hundredtbs sec.) } & \multirow{2}{*}{ Diff. } & \multirow{2}{*}{$\begin{array}{l}\text { Std. Error } \\
\text { Mean Diff. }\end{array}$} & \multirow{2}{*}{$t$} \\
\hline & GA & LA & & & \\
\hline$/ \mathbf{i} /$ & 12.90 & 8.10 & 4.80 & .520 & $9.23^{*}$ \\
\hline /e/ & 14.45 & 15.80 & 1.35 & .600 & $2.25 t$ \\
\hline$/ a /$ & 20.00 & 13.05 & 6.95 & .715 & $9.72^{*}$ \\
\hline 101 & 17.55 & 16.85 & 0.70 & .746 & 1.00 \\
\hline$/ \mathrm{u} /$ & 16.35 & 12.30 & 4.05 & .383 & $10.57^{*}$ \\
\hline
\end{tabular}

* Indicates significance at $1 \%$ level with 8 d.f.

† Indicates significance at $5 \%$ level with 8 d.f.

tempts at speaking foreign languages are likely to be characterized by sound duration differences contributing to "American dialect."

\section{CONCLUSIONS}

Not much remains to be said by way of conclusion, since each subsection of the paper ended with a summary statement. In my opening remarks I suggested that the research evidence I planned to present might lead you to share with me the conclusion that failure to take time factors into account. when speech analyses are performed can result in serious error. I hope this point has been established, and with it the conclusion that time research and the application of these research results may contribute materially to improved language teaching.

\section{REFERENCES}

1 Draegert, G. L. "Relationships Between Voice Variables and Speech Intelligibility In High Level Noise," Speech Monographs, Vol. XVIII, No. 4, November, 1951.

2 Hanley, T. D. "An Analysis of Vocal Frequency and Duration Characteristics of Selected Samples of Speech From General American, Eastern American and Southern American Dialect Regions," Ph.D. Dissertation, State University of Iowa, August, 1949.

8 Murray, E. and Tiffin, J., "An Analysis of Some Basic Aspects of Effective Speech," Archives of Speech, Vol. I, No. 1, January, 1934.

4 Navarro Tomás, T., Manual de Pronunciación Española, Quintana, Madrid, 1926.

6 Tiffany, W. R., "Vowel Recognition as a Function of Duration, Frequency 
Modulation And Phonetic Context," "Journal of Speech and Hearing Disorders," Vol. XVIII, No. 3, September, 1953.

- Tiffin, J. and Steer, M. D., "An Experimental Analysis of Emphasis," Speech Monographs, Vol. IV, No. 1, December, 1937.

\section{Discussion}

There was a discussion after Professor Hanley's paper.

ROBERT STOCKWELL said: The last charts presented comparing the duration of certain vowels in Spanish and English would suggest some way of controlling the conditions under which these vowels were measured. For example, were all the " $a$ " vowels measured under what might be called Primary Stress in both languages, or was this done on an average? And if it was done on an average, then of course there are certain difficulties because English has four levels of stress and Spanish has only two. Your average figures will not work out usefully for pedagogical purposes under these conditions. And the second question is that of stressed and unstressed vowels, especially in measuring English vowels. You talked about a sentence concerning Mr. Roosevelt, and you said that they changed the stress on each word in the sentence as read five times or as many times as there were words in the sentence. Now the unfortunate thing in such an experiment is that you have to consider the fact that English does not have $a$ stress or $a$ strong stress or $a$ weak stress. It has four clearly demarcated levels of stress and it would be impossible to remove at least the secondary level of stress from some of the words when you put the stronger stress on another word. Consequently you might be comparing in one word a weak stress with the same item under strong stress, whereas in the next word when you said it was longer or shorter with its heavier stress, you might actually be comparing a secondary level of stress against a primary, and therefore not have comparable data at all in your different experiments on that level.

T. D. HANLEY: With regard to the last point, perhaps it was not clear that each word was compared only with itself, under the different conditions of the experiment.

EINAR HAUGEN: There was something I didn't catch-you had these vowels with various durations under different conditions running from 6 centiseconds to 50 centiseconds. How were those differences in duration produced? Did you cut them off or did you extend them, or did you blow them up? 
T. D. HANLEY : They were cut down from prolonged vowels.

HAUGEN: By what technique? Do you mean by actually slicing off pieces?

HANLEY: Yes, by scissoring off the tape.

HAUGEN: But that would obviously change your vowel, you have not then got the same vowel.

HANLEY: That's true.

HAUGEN: Therefore I don't see the relevance of these experiments. You definitely changed the quality. Because if you take a vowel /ey/ you are starting out with one vowel and ending up with another-it's not strange that some people thought it was /i/ if what they heard was simply the end of it, and on the other hand if it was the beginning, one would not be surprised if it were heard as /e/ or something in that line. So I am wondering how you provided constant vowel conditions.

HANLEY: The point is well taken, particularly with regard to the diphthongized American vowels. That study was one in which I did not participate. I know only the published results. Specificity with regard to the cutting technique was not described. I can speak specifically with regard to the Latin American vowels in which I participiated, and I fully agree that there was an artificial aspect to the situation. Whether it can be demonstrated in future research that under some other method of producing the shortened and lengthened vowels the same results would obtain, I don't know. All that I hold for these results is that they are reliable, that is, they tend to be reproduced among different people, among different listeners. There is a tendency to make the same kind of response to these stimuli.

STOCKWELL: Is there some reason why you didn't use real samples of real speech and make the same test?

HANLEY: The difficulty of controlling all other factors by using real samples of real speech so that we could say "Only the time is the element that is being manipulated here," was technologically beyond me at the time the experiment was conducted-and is still beyond me.

VINAY (Montreal): I have always found in measuring time that it is extremely difficult to find boundaries according to which you could measure time. You all know Prof. Menzerath's experiments on coarticulation and I found the same thing in trying to apply time measurement to samples in French. For instance, it is very hard to know 
when to begin to measure something, and when to end your measurements, so I was hoping you might say something about this aspect, in view of the fact that in some languages apparently when you measure time-if you go only by the acoustic samples-you are not measuring the whole thing. I remember when I was working with Professor Jones in London they were measuring strongly stressed statements in English and they found that in the words "Thank you" you could have a long duration in the "Thank" although the word "thank" was actually not pronounced so that the duration would be on something which was not said, in which case the boundary would have to be extended far back before the first speech unit begins.

HANLEY: I should like to reply to that by stating that on the two studies for which tables were provided in the slides, the measurement of time was not a critical factor, since the stimuli were produced by the rigid method of cutting the tape. In the emphasis study, the time measurements were made by means of the phonophotographic technique, the pitch analysis technique, which was previously shown. The study that was involved in time as a measure of excellence or contributing to excellence, also made use of the phonophotographic technique. There they were not, you will recall, working with individual sound units in terms of speech sounds, but durations of phonations, and I think your criticism there would not apply since that is an all or none proposition. 


\section{Language and Culture}

\section{Introductory Remarks}

Mrs. Regina Flannery-Herzfeld (Catholic University): I would like to make a few remarks on the main speakers. The field of language and culture studies is a new and somewhat controversial one. It is generally recognized, of course, that language is a most important part of culture. As it has recently been phrased, "Speech has relatively high autonomy, but culture could probably not originate without language and language would be nearly empty without culture." In exploring the actual relationship of language systems to other cultural systems a number of problems on the borderline between linguistics and cultural anthropology are raised. Just as with another new development (that of personality-culture study) there is no agreement as to just what sort of study should be undertaken by specialists in the component disciplines. In the area of language and culture, however, the linguists have the advantage, providing they have the interest, as a solution, or even the posing of the important problems, requires a mastery of linguistic techniques which few cultural anthropologists possess. A variety of approaches to the study of language and culture have been made. We are fortunate in our panel today, as the speakers will present some interesting new approaches. While Dr. Politzer and Dr. Welmers are both concerned with foreign language study in relation to culture, each has chosen a quite different facet. Dr. Politzer will emphasize the depth of cultural understanding that can be developed only through the language itself as distinct from the kind of understanding to be derived from reading text translations, even though these be accompanied by descriptions of the culture. On the other hand, Dr. Welmers is concerned with quite a different aspect. $\mathrm{He}$ will relate non-segmental phonemes to the universal world of sound, and draw some interesting conclusions from the implications.

The other two papers deal with the problem of change; Dr. Olmsted will present a theory of linguistic change that takes into account both linguistic and non-linguistic factors, a theory that may be as interesting as it seems. Dr. Garvin will draw on his experience, like Dr. Olmsted, in an area of non-Indo-European speech, in introducing a type 
of cultural change analyzing both the linguistic and the cultural factors involved in the situation. I would like, then, to ask Dr. Politzer to present his speech:

\title{
Developing Cultural Understanding through Foreign Language Study
}

\author{
ROBERT POLITZER
}

\section{Harvard University}

Last summer an interdisciplinary seminar in Language and Culture was held in Ann Arbor under the auspices of the Modern Language Association. I was fortunate enough to be a member of this seminar and a cosigner of its report which appeared under the title "Developing Cultural Understanding through Language Study" in the Publications of the Modern Language Association (Vol. LXVIII, No. 5, pp. 1196-1218). Obviously I shall not attempt to repeat or even summarize this report at this occasion. What I shall rather do is to comment briefly on the connection between cultural analysis and language teaching as it seems to be envisaged by the report and then try to discuss and answer the reactions to the report, whichafter long personal discussions with some of my colleagues and after the official panel discussion of the report at the last meeting of the Modern Language Association-seem to me the most significant and most noteworthy.

Our seminary intended to investigate the claim that language study leads to cultural understanding, and the means by which such cultural understanding could be achieved. Cultural understanding was defined mainly as an awareness of the nature of culture (e.g. learned and shared behavior) and as the attainment of a non-culture-bound attitude. By the latter, it seems to me, we meant the relativistic attitude of the Social Scientist (though the word "Relativism" as such does not appear in the Seminar Report). The conclusion at which the Seminar arrived was the following: In order to combine the teaching of language with that of cultural understanding, we must make a precise analysis of American culture and of the culture of the 
country whose language we want to teach. This analysis must be carried out with the precise tools of the social scientist (using concepts like theme, culture-trait, etc.) and in the spirit of social relativism. The comparative cultural analysis, supplemented by special studies like those of national stereotypes, will furnish us the items of similarity and difference between the two cultures which-again in a spirit of social relativism and objectivity-should be presented to the students or upon which the reading or discussion material in the language classroom should concentrate.

In discussing the reactions to our report, I shall not deal with those which are either due to misunderstandings or which are in a sense inanswerable, and lead only to philosophical discussion. To the first category belongs the-fortunately very rare reaction of the professor of French literature who claims that Racine and Corneille are the best representatives of French culture-thus giving the word "culture" a meaning different from the one explicitly stated in our report. To the second category belongs the query whether freedom from "culture bondage" and the implied relativistic position is a desirable educational objective or whether the relativism of the social scientist is a tenable philosophical position. So, leaving aside misunderstanding and the discussion of philosophical ultimates, let me turn to the first reaction. This is the comment of a Professor of French literature who claims the following: "I am teaching in a Department of Language and Literature; my aim in teaching language is to prepare students for the study of literature which will give them access to the cultural achievements of France-cultural achievements in terms of esthetic and moral absolutes. I understand the social scientists' definition of culture very well indeed, but never having made the claim to impart the cultural understanding described in your report, I am not interested in your subject or in the connection between culture and language". Now it is, of course, perfectly true that cultural understanding as an aim of language instruction may be a personal choice-but the connection between language and culture-and this is a point which I believe became clear to us during the seminar discussions-is inevitable. As language teachers we must be interested in the study of culture (in the social scientists' sense of the word) not because we necessarily want to teach the culture of the other country but because we have to teach it. A language is after all only a system of arbitrary 
symbols by which the members of a speech community communicate. It is a code which receives meaning only because it refers to objects, concepts, or activities which exist in that particular community. If we teach language without teaching at the same time the culture in which it operates, we are teaching meaningless symbols or symbols to which the student attaches the wrong meaning; for unless he is warned, unless he receives cultural instruction, he will associate American concepts or objects with the foreign symbols. Ultimately the very understanding of literature depends upon understanding the meanings - and this means the cultural context-in which the language symbols are used. Thus we might say that the relation between language and literature is vertical (the understanding of literature presupposing the understanding of language) and an optional one as far as the curriculum is concerned, while the relation of language and culture is horizontal (language cannot be understood without cultural context) and a necessary and inevitable one.

The second attitude I want to consider is that of a linguist who feels that (1) cultural analysis and cross-comparison is beyond the necessary scope of his teaching and who remarks that (2) as a matter of fact the very idea of a systematic comparative analysis of the two cultures seems to be borrowed from the scientific approach toward language teaching, which makes a comparative analysis of the two languages. Now as far as the first remark is concerned what applies to the professor of literature applies also to the linguist: the necessity of teaching meaning makes the study of culture imperative. As to the second objection which implies that a comparison of linguistic systems is a sufficient basis for language instruction, let us take a closer look at the way in which linguistic systems can be compared: if we compare let us say English /p/ and French /p/ we cannot compare them as phonemes but only as phonetic realizations, we compare phonetic substance, as phonemes (this means from the point of view of function within their system) they are incomparable. If we compare English I want him to do it with French Je veux qu'il le fasse we are comparing two statements which become comparable only because they mean the same. In other words, a language is the association of sound and meaning in a system of symbols, but the basis of comparison between systems rests either in the realm of sound or in the realm of meaning (on the prelinguistic and metalinguistic level as some lin- 
guists would say). But if we compare two structures because they mean the same, we must of course ask the question, "how can we be sure that they have the same meaning?" This is not the place to revive the discussion of the meaning of meaning, but certainly one of the most important objective ways of ascertaining identity in meaning of two utterances would be to determine that the speakers of the two different speech communities use the utterances to refer to the same objects, that they are responses made in the same or similar situations. In other words, we are back at the necessity of examining cultural contexts. It is interesting to note, incidentally, that the very linguists who defined meaning most precisely and sharply "out of language", were and are inevitably among those who pioneer in the field of comparative cultural analysis when it comes to language instruction (see e.g. E. T. Cornelius Jr., Language Teaching, New York 1953, Ch. IV; E. T. Hall and G. L. Trager, The Analysis of Culture, Washington D. C. 1953).

The third criticism comes from a Social Scientist: "I agree with the idea of comparative cultural analysis and I fully agree that a knowledge of the foreign culture is a prerequisite for the knowledge of the foreign language. But if cultural understanding is the aim, why not present the student simply with the results of the comparative cultural analysis; in other words, why not simply lecture to him in English on the nature of culture, on cultural differences, relativism, etc. and take your objective by the frontal attack."

There are several possible answers to this suggestion. The first and most obvious is that language is not only a key to culture but is itself part of culture, learned and shared behavior par excellence. The student who talks or learns about a community without paying attention to its language simply ignores an important part of its culture. Language is also that part of culture with regard to which we are apt to be most "culture bound." The student who learns the most obvious lesson, namely that there are different ways of expressing the same ideas or concepts in different languages, learns inevitably the lesson that the important segment of culture, called language, is based on convention. In addition the language student will also almost inevitably become aware of the fact that different languages divide reality differently: to give an example, that segment of reality which in English is referred to by wood and forest, is in Spanish divided into leña, bosque, silva, 
madera, and to quote again de Saussure's famous example, French mouton is English mutton as well as sheep. (F. de Saussure, Cours de linguistique géneral, 2d ed. Paris, 1922, p. 160). All this is a practical lesson in cultural relativism. Another argument in favor of language study as a means of achieving cultural understanding-which was incidentally briefly mentioned in the seminar report (p. 1213)-rests upon the assumption that different languages do not only describe reality in different terms but as a matter of fact change the view of reality, the appearance of reality itself. This attitude which has its own continued history reaching from at least W. Humboldt to B. L. Whorff, has recently again become an object of investigation and discussion (see for instance S. Öhman, "Theories of the "Linguistic Field" ", Word IX (1953) 123-135, Eric P. Lenneberg "Cognition and Ethnolinguistics", Language XIX, (1953), 463-472). Here I should only like to stress the importance of ethnolinguistic investigation from the point of view of our pedagogical claims in favor of language instruction as means of imparting a less culture bound attitude.

A person who learns about the culture of another country in terms of his own language will inevitably look at the foreign culture from without, a person who learns to use the foreign language has at least a good chance to assume a position from within. A student who learns that the French language has a term "civilisé" which has no exact counterpart in English because it implies a great number of qualities, has learned a cultural fact about France; but a student who has learned to use the word "civilise" correctly and without hesitation in French has learned-at least in this one respect-to assume the point of view of a Frenchman.

The argument of language as a key to cultural understanding, it seems to me, rests largely on a distinction between two types of learning, and of knowledge: learning and knowledge of things and knowledge not of things but about things. The first type of knowledge is acquired by doing and by direct contact; it is the type of knowledge that is gained in the laboratory. The second is acquired not by doing but by being told and it is imparted in the lecture room. Now it is interesting to note that the pragmatic American educator is, of course, apt to class language study with the second type-namely knowledge of symbols rather than facts--knowledge "of" and acquired through speech rather than action. But the important point to be emphasized 
is precisely that when it comes to teaching of cultural understanding, language study belongs in the first category rather than in the second. The student in the language class uses and lives foreign culture-the student in the anthropology class hears about it. This is not meant to depreciate classes in anthropology, but if we believe in learning through doing rather than learning through "talking about", then paradoxically enough the language classroom should be an ideal place for the acquisition of non-culture bound attitudes.

There is yet one more attitude toward the problem of achieving cultural understanding through language study upon which I should like to comment. It is the attitude of persons whom I should like to refer to simply as conscientious language teachers. Their comment is the following: "Preoccupation with so-called cultural objectives, while acceptable in theory, is practically always harmful in practice because it detracts from our efficiency in teaching the language skills. As a matter of fact, it happens only too often that bad language teaching hides behind precisely such claims as developing cultural understanding." I should like to register complete agreement with that attitude. The necessity of examining and teaching culture with language, the possibility of imparting cultural understanding through language study should never be used to disguise failure to teach the language. There is of course a great temptation to defend language study by saying: I realize that my students didn't learn much French, but think of all the cultural understanding they acquired! Frankly, I think that this claim will never stand up under closer scrutiny; for the claims of the language teacher to impart cultural understanding beyond that or different from that imparted by the social scientist rests largely on the distinction between doing and talking about-and doing in this case means using the foreign language, and using it well. The ethnolinguistic argument that different languages are different ways of looking at the world, proves as such nothing with regard to the student, for he may very well have studied other languages without having come to the personal realization that this is so: If he reads a page of French and this page of French means nothing to him until he has slowly deciphered it and put it into English, then the French text will "fade away" and only the English translation will remain. The student will think of the ideas, the concepts on that page from now on as English concepts, English ideas. If this is his approach to the foreign 
language, then I fail to see the important specific contribution which language study has made to his cultural understanding. In the same way, if he can respond to the stimulus of a situation only in terms of his native language, language study has made no specific contribution to his cultural understanding, even though the student may be able to translate--by means of mechanical rules, learned vocabulary equivalents, etc.- his response into another language. Only if the student knows the language well, if he can react immediately in the foreign language, has language study made its important specific contribution. For being able to react immediately in two languages means to be capable of two different responses each of which is normally at least determined by a different cultural complex. It is in this immediate, self-experienced lesson in cultural relativism that $I$ believe the main contribution of language study to cultural understanding must ultimately rest. So, far from detracting from the study of language skills, the claim of imparting cultural understanding through language study rests-in the final analysis-largely on effective language teaching.

\title{
Towards a Cultural Theory of Lexical Innovation: A Research Design
}

\author{
D. L. OLMSTED
}

\section{University of California, Davis}

Anyone who has engaged in as much criticism of other people's theories as I have must eventually face up to the task of constructing one himself. If this activity accomplishes nothing else, it may at least serve to impress upon him the difficulty of theory-construction about human behavioral data and make for a tempering of his vituperative and polemical energies.

As usual, I would like to begin by defining the items in the title of this paper. The first word, "towards", is included to indicate the inchoate state of these formulations; the postulates presented here are perhaps more likely to turn out to be necessary than to be sufficient. By "cultural" we mean to specify the data which are the object of 
the theory as human learned behavior, both linguistic and nonlinguistic. By "theory" is meant, as usual, a set of statements consisting of the following kinds: one or more postulates, formal definitions indicating the use of terms in the postulates, transformation rules for the postulates, and one or more theorems deduced from the postulates by the transformation rules.

"Lexical" is meant to indicate interest in the vocabulary of a language. We do not seek to predict anything about the phonemes of the language or about anything except the fate of new words; "innovation" is said to occur whenever any speaker $y$ adopts in otherwise regular utterances of his language some new word previously used by some other speaker $x$. Note that the theory is not concerned with coining of words, only with their acceptance and spread among speakers. We assume a word as already coined and used by at least one speaker before the theory is operable. It follows from the above that the theory is not restricted to the first use of any word in any language, but ought also to deliver predictions about the spread of a term from any speaker to any other speaker in the same speech-community.

As will be evident below, prediction of lexical innovation cannot rest on linguistic postulates alone. A lifetime of analysis of linguistic corpora will not tell us why a speaker adopts one form and rejects another, though $I$ hasten to add that such analysis is a necessary condition, even though not a sufficient one. For the rest of the answer it is necessary to consider such variables as the speaker's learning history and position in society and also the non-linguistic correlates of the word.

In consideration of these other variables an effort has been made to secure the best postulates and techniques possible from three other disciplines: anthropology, sociology, and psychology. None is, of course, acceptable to all experts in those fields, but each notion or method utilized here follows competent precedent in the three disciplines. Nor can it be claimed that the selection of the variables is a new development. On the contrary most of the variables are ones that have been suggested by workers in historical linguistics or dialect geography, though almost always in crude and untestable form.

If there is anything new here about these variables, it is their explicit statement in a single series of postulates so that they can all be brought to bear in a single situation, tested for their relative con- 
tributions to the work of predicting behaviorial events, and discarded, modified, or supplemented as may prove advantageous.

The sub-title, "research design", has been added in the belief that a theory unaccompanied by the operational specifications necessary to render it testable is a mere speculative exercise.

The definitions and postulates will be presented first, then the operational problems involved in the field research will be discussed, together with a few of the possible theorems to be tested.

\section{Preliminary Postulates and Definitions}

0.1. Among the utterances of the members of at least some speech communities there is at least one free form which has never been used by at least some member.

0.2. Def. Such a free form is for the present purposes to be called a test word.

0.3. Def. The class of speakers in whose idiolects the test word is occurrent are models.

0.4. Def. The class of speakers in whose idiolects the test word is nonoccurrent are tories (Irish tōraidhe "pursuer").

1. The likelihood that some tory $y$ will adopt a test word used by some model $x$ is:

1.1. An increasing function of the phonemic regularity $R_{p}$ of the test word.

1.2. Def. $R_{p}=\frac{F_{i}+F_{j}+F_{k}}{n}$, where $F_{i}$ is the frequency of the initial phoneme or cluster (either one or more vowels or one or more consonants), $F_{j}$ is the frequency of the least frequent sequence of three or less medial phonemes, $F_{k}$ is the frequency of the final phoneme or cluster (either one or more vowels or one or more consonants), and $n$ is the number of words in the corpus in which the $F$ 's are calculated. ${ }^{1}$

${ }^{1}$ Floyd Lounsbury has pointed out that phonemic regularity might be more satisfactorily measured in terms of the average transitional probability obtaining between the phonemes in the test word. Such a measure would involve a further research step of no mean magnitude-the calculation of such probabilities for the language as a whole. The measure proposed here is retained, provisionally, in the belief that around zero the two methods would not differ greatly in results and that above that figure the differences decrease in importance. Ultimately, however, Lounsbury's proposal seems preferable. 
2. The likelihood that some tory $y$ will adopt a test word from some model $x$ is:

2.1. An increasing function of the morphemic regularity $R_{m}$ of the test word.

2.2. Def. $R_{m}=\frac{F_{d}+F_{d}}{F_{a}}$, where $F_{d}$ is the frequency of any inflectional affix occurring with the test word and $F_{e}$ is the frequency of the most frequent affix of the same class. Affixes whose (non-contrastive) distribution is predictable on phonemic grounds are considered the same affix. (This insures against counting phonemic irregularity twice.) $F_{f}$ is the frequency of any derivational morpheme or the sum of the frequencies of any derivational morphemes, which are parts of the test word. ${ }^{2 b}$

3. The likelihood that some tory $y$ will adopt a test word from some model $x$ is:

3.1. An increasing function of the positive difference in status, $S_{x / y}$, of $x$ over $y$.

3.2. Def. $S_{x / y}=\frac{S_{x} / S_{y}}{N}$, where $S_{x}$ is the status of $x, S_{y}$ is the status of $y$, and $N$ is the total number of members in the speech community. ${ }^{2 a}$ 3.3. $S_{x / v} \equiv 0$.

4. The likelihood that some tory $y$ will adopt a test word from some model $x$ is:

4.1. An increasing function of the upward mobility, $M$, of $y$.

4.2. Def. $M=S_{y}-S_{p}$, where $S_{v}$ is the status of $y$ and $S_{p}$ is the average of $y$ 's parents.

5. The likelihood that some tory $y$ will adopt a test word from some model $x$ is:

5.1. An increasing function of the frequency of interaction, $F_{I}$, between $x$ and $y$.

5.2. Def. $F_{I}=\frac{I}{w}$, where $I$ is the total hours of interaction, $w$ the number of weeks.

6. The likelihood that some tory $y$ will adopt a test word from some model $x$ is:

3a It will probably be wise, in preliminary tests, to restrict the sample to members of one sex.

${ }^{2 b}$ Cf. Dwight L. Bolinger, "Shivaree" and the phonestheme. American Speech, 25, (1950) 134-135. 
6.1. An increasing function of the frequency of occurrence of the test word, $F_{a}$, in the idiolect of $x$, during interaction with $y$.

6.2. Def. $F_{a}=\frac{F_{b}}{F_{I}}$, where $F_{b}$ is the average number of occurrences per hour.

7.1. Def. The likelihood that some tory $y$ will adopt a test word from some model $x$ is $L$.

7.2. $L=\left({ }_{a} R_{p}\right)\left({ }_{a} I\right)\left({ }_{f} F_{a}\right)\left({ }_{b} R_{m}+{ }_{a} S_{x / y}+{ }_{a} M\right)$.

7.3. Def. $a, b, c, d, e$, and $f$ are empirical constants, whose values are unknown at present, which assist in the proper weighting of the variables (cf. 13.1 below).

\section{Research Problems.}

The first problem in the testing of the theory is the selection of a speech community. It is obvious that some at least of the above postulates could be tested by deriving theorems that would apply to a laboratory or quasilaboratory situation. Such investigation may indeed prove the most convenient way of testing some of them. But it is also of considerable importance to construct theories in such a way that they may apply to natural languages spoken by members of actual societies. Importance was therefore attached to study of the variables in an extant society under field conditions.

It was clear in the light of this requirement that the community should be small enough to permit of observation of all its members in order to ensure that a new item might not be learned from some model not subject to investigation. This ruled out, of course, any investigation of a community that is only a part of a larger speech-community; e.g., a small community of English-speakers, no matter how isolated, would still be susceptible to untraceable influences from press, radio, or occasional interactions with outsiders. Since the study necessary for making an actual prediction must be carried on over a period of years, the group had to be accessible, although of course such a group as Holmberg's Sirionó would have been better, because more isolated. The group that seemed best to meet these requirements was the Achumawi ${ }^{3}$ of northeastern California. There are less than fifty speakers of Achumawi alive today.

Grateful acknowledgement is made to Professors A. L. Kroeber and M. B. Emeneau who first suggested the Achumawi and supplied assistance and encouragement with respect to field work. 


\subsection{The Test Word}

9.1. The first consideration concerning the test word is its accurate operational specification, i.e., making sure a given item is used by some speakers but not by others. The best way to ensure that a speaker has not used a word is to be sure that he has never even heard it. Two ways suggest themselves: observation of the community at a period when a new term is introduced naturally by the appearance of some new item ${ }^{4}$ of non-linguistic culture, or coinage and introduction through trusted informants of test words devised for the occasion. The second method has several advantages. First the source is unambiguously known and models can be chosen so as to control for status, amount of interaction, etc. Second, both phonemic and morphemic regularity can be controlled. Third, the timing of the whole series of operations can be controlled.

9.2. The calculation of measures of phonemic and morphemic regularity requires that linguistic analysis of the phonemics and morphemics be done first. Then must come the frequency counts. The method here proposed for calculating phonemic and morphemic regularity is quite tentative, and if a preferable one is proposed, it will meet with ready acceptance.

10.0. Rank Determination

10.1. Social stratification is the process of ranking individuals within societies. Much of the controversy ${ }^{5}$ over social stratification has re-

${ }^{4}$ Elmendorf has described with admirable precision the custom of word taboo among the Coast Salish. The occasion of the taboo of any word should afford a good chance to observe the fate of its successor. Cf. William W. EImendorf, Word taboo and lexical change in Coast Salish. IJAL, (1951), 17, 205208.

${ }^{5}$ Cf. Reinhard Bendix and Seymour Martin Lipset (editors), Class, Status, and Power, A Reader in Social Stratification, Glencoe, (1953); Talcott Parsons, An analytical approach to the theory of social stratification, Amer. J. Sociol., (1940), 45, 841-62; Hans Speier, Social stratification in the urban community, American Social. Rev., (1936) 1, 194-5; W. Lloyd Warner and Allison Davis, A comparative study of American caste, in Edgar T. Thompson (ed.), Race Relations and the Race Problem, (1939), Durham; Kingsley Davis, A conceptual analysis of stratification, Amer. Sociol. Rev., (1942) 7, 3, 309-21; W. Lloyd Warner et al., Social Class in America, (1949) Chicago; Maryon K. Welch, The ranking of occupations of the basis of social status, Occupations, (1949) 27, 237-41; Ralph Linton, The Study of Man, (1939) New York; August B. Hollingshead, Elmtown's Youth, (1949) New York; George P. Murdock, Social Structure, New York (1949); Robin M. Williams, American Society, A Sociological Inter- 
sulted from failure accurately to delimit several dimensions of the problem: (a) formal definition of social class or of caste (b) the locus of the group taken as the object of the investigation, (c) operational specifications of social class (or caste or rank), and (d) other variables correlated with class, caste, or rank. Each of these dimensions is discussed below; in so doing, the term applying to the cluster of statuses -whether they be of class or caste nature-characteristic of the individual will be used. This term is "rank."

10.2. The problem about formal definitions in social stratification has centered around the question, "How many social classes does society X REALLY possess?" There generally follow discussions of real vs. nominal definitions in which the number of morphemes is inversely proportional to the competence of the writer. Such "REALLYpossess" questions are, of course, totally spurious for any scientist who has discovered that language is made up of conventional signs. The reason for this is that at the root of such questions lies the assumption that there is some utterance that must have some empirical referent in the world, once and for all time, and that the problem is to discover. what that referent is. The scientist, on the other hand, is only interested in whether a definition is useful in helping him discover the grand regularities that are presumed to characterize men and nature. For example, the definition, "a social class is a set of Chinese Popes" is not a useful definition since it asserts that a social class is a set consisting of no members and thus gives very poor instructions about where to go and what to observe or manipulate if the object of study is a social class. The view taken here is thus the nominalist one with utility judged roughly by the ratio between the amount of in-

pretation, (1951) New York; Richard Centers, The Psychology of Social Classes, (1949) Princeton; Some of the previous attempts to apply social stratification theory to individual communities are: Robert S. and Helen M. Lynd, Middletown, A Study in Contemporary American Culture, (1929) New York; W. Lloyd Warner and Leo Srole, The Social Systems of American Ethnic Groups (1946) New Haven; W. Lloyd Warner and Paul S. Lunt, The Social Life of a Modern Community, (1941) New Haven; James West, Plainville, U. S. A. (1945) New York; Hollingshead, op. cit.; Alfred Winslow Jones, Life, Liberty, and Property, (1941) Philadelphia; Elin L. Anderson, We Americans, A Study of Cleavage in an American City (1937) Cambridge; John Dollard, Caste and Class in a Southern Town (1937) New York; John Useem, Pierre Tangent, and Ruth Useem, Stratification in a prairie town, Amer. Sociol. Rev., (1942) 7, 3, 331-42; Allison Davis, B. B. Gardner, and M. R. Gardner, Deep South (1941) Chicago. 
formation needed empirically to interpret any definition and the amount of information that can be predicted given the knowledge that a datum fits the definition. 'To put it another way, if our definition of "social class" is such that it is easy to determine what class Mr. Arbuthnot belongs to, and if knowledge of his class, so defined, allows us to predict all that is of interest concerning his other qualities, the definition is useful.

10.3. The locus of the group of individuals to be ranked is a question on which students of stratification are divided, some maintaining that it should be restricted to the individual's face-to-face interaction group, others contending that the entire society, made up of a multiplicity of such interaction groups, is the better unit, while still others settle for a unit of intermediate size, such as a city like those designated by the pseudonyms, "Middletown," "Yankee City," "Southerntown," "Elmtown," and "Plainville." These differences of opinion reflect different problems: investigators favoring smaller units are often concerned with "group dynamics," while those favoring larger units are sometimes interested in the economic aspects of the classes so defined. In our case, of course, the unit that suggests itself is the speech community. Care was taken to choose a group for whom the society (defined ethnically) would be substantially identical with the face-to-face interaction group, ${ }^{6}$ thus obviating difficulties on this point.

10.4. Operational specifications of stratification have taken many forms, from "objective" measures like income, formal education, and house type to "subjective" ones such as the subject's own ranking of himself-a measure, incidentally, that has proved surprisingly useful for certain purposes. Somewhere between these methodological extremes lie the data gained by asking some subjects to rank others. These latter data have the virtue from the standpoint of lexical innovation, that they are actual differentiating behavior by some members of society toward others and thus presumably stem at least in part from factors that also influence lexical acceptance. They are more

"Achumawi do interact somewhat with English-speaking "Anglos," and they do form, in some respects, part of the larger American society, so that the cloth is a little ragged on both ends, although the assumptions that have to be made about the sample remain simpler than those of most other social stratification studies. 
likely to stand the test of cross-cultural comparison than such measures as income, which may be reckoned in dollars, drachmas, cattle, or cocoanuts, or formal education, for which the society may thoughtlessly have made no provision. The same advantage appears a fortiori with respect to subject's rankings of themselves, which are obviously greatly dependent on what Bloomfield might have called tertiary responses to social stratification, i.e., the society's own folklore (accurate or not) with respect to its organization.

Sociologists ${ }^{7}$ have had good results with the data obtained from selected respondents who rated all other subjects. Selection of the raters obviously requires the degree of knowledge of the society which has come with decades of research on our own society; such conditions are not likely to be duplicated when attention is turned to other less well-known societies.

For these reasons, the technique known as sociometrics recommends itself. It involves rankings of every member of a group by every other member, thus eliminating selection of raters by the investigator. There are no limits to its application in any culture except size of group. For these reasons it seems promising as a method for filling the blank in the equation reserved for ranking individuals along a continuum of esteem. ${ }^{8}$

The chief disadvantages of sociometric techniques are two: for reasons intrinsic to sociometric theory, they can only be used with groups of small size, and their use threatens rapport with informants.

The Achumawi were chosen partly because their dwindling numbers allow sociometry to be used, partly for the interest in their language, a study of which ought to result even if the rest of the research program should come to nought.

Finally, it should be noted that this particular technique for social stratification represents only one guess as to how to get at the variable noted by linguists when they coined such terms as "hyper-urbanism"; it is hoped that at least some other methods could be substitued without forcing major changes in the theory.

'E.g., Hollingshead, Elmtown's Youth, op. cit.

"Power" is explicitly not provided for, though it is undoubtedly important in some of the problems of social stratification. The assumption here is that it is significantly less important for the present purposes than esteem. 
11. The measure of upward mobility ${ }^{9}$ is intended to get at the individual tory's tendency to imitate those ranked above him, since sociologists have shown ${ }^{10}$ that any individual's chances of making a successful move to a higher social stratum are fairly directly proportional to his capacity to adopt the behavior patterns characteristic of that stratum. ${ }^{11}$ The particular one chosen has the disadvantage that it can be used only with adults. Furthermore, some corrections might have to be made in societies where a great premium is put upon age as a factor making for esteem.

In other respects the measure seems relatively well adapted to cross-cultural comparability, and has the added advantage that it is independent, or nearly so, of the measure of rank.

12. In order to calculate the frequency of interaction (cf. 5.12) and the frequency of occurrence of the test word, it is proposed to spend as much time as possible with the tory in question for a period of a month, say, in order both to get these measures and, in addition, to ensure that no other model uses the test word in interaction with the tory.

13. A final word is perhaps in order with respect to the form of the equation..$^{12}$ The specific form, of course, must remain a guess at this stage of research. What is needed is investigation of each of the variables, other things being held constant, before the final form of the equation can be ascertained. However, it is both tempting and useful to speculate on the mathematical relations obtaining between the variables.

13.1. The tentatively final form of the equation contains constants $(a, b, c, d, e, f)$ multiplying the numbers for the variables. These constants are included to provide against undue weighting of one variable or another depending on the essentially arbitrary measures chosen for the variables; e.g., if amount of interaction were measured in hours per day instead of hours per week, the denominator of the fraction would be 30 instead of roughly 4 , over a month's span. The constants thus provide some slack in the theory which should be taken up

9 I am indebted to A. B. Hollingshead for suggesting the form of this measure.

${ }^{10}$ Hollingshead, op. cit.; Davis and Dollard, op. cit.

11 It is not denied that other factors are also operative in upward mobility.

${ }^{12}$ I am greatly, indebted to Lloyd Morrissett for his suggestions on this point. He is, of course, not responsible for any errors in the form adopted. 
when empirical work has made possible the assignment of values to the constants.

13.2. The equation expresses some relations of variables as multiplicative, others as additive. At the moment this indicates only our hunch about the relative importance of the variable. If (a) phonemic regularity, or (b) interaction, or (c) frequency of test word, or (d) the combination of morphemic regularity, rank differential and mobility, -any one of (a), (b), (c), or (d)-goes to zero, these will be no lexical innovation whatsoever. In other words, the prediction is that there will be no lexical innovation if the following circumstances obtain: if the test word contains a foreign phoneme sequence or a nonoccurrent sequence of native phonemes, ${ }^{13}$ or, if there is no interaction between model and tory, or if there is no occurrence of the test word in the model's utterances while interacting with the tory. ${ }^{14}$ To put the matter another way, the indispensables for lexical innovation are pronounceability and opportunity.

${ }^{13}$ It follows from this prediction that word-borrowing involving foreign phonemes (e.g., the adoption by the Zulus of clicks from Khoisan) can never come through a single bilingual speaker. If the test word contains foreign phones, borrowing may take two forms: (a) substitution of native phones for foreign ones, and (b) relatively close retention of the foreign pronunciation. It is likely that no lexical innovation takes place from a single bilingual model that involves close retention of the foreign pronunciation. Thus it follows that the adoption by the Zulus of Khoisan clicks must have required not one but a number of bilingual models. For a single model would probably not provide a constant enough source of stimulation to the tory and phonetic substitution by the tory would often go unnoticed, unpunished, and hence uncorrected. A number of bilinguals, however, might, if they constituted an important segment,- perhaps a majority would be needed-of the tory's interaction group, confront him at every turn with the "correct" foreign pronunciation of the word. The ideal tory in this situation would be the one that was most teachable and the one that had the greatest opportunities to interact with the models, for example, a child of mixed parentage. A generation of such children, if they spoke their fathers' language-Zulu-with some unchanged borrowings from their mothers' tongue-Bushman or Hottentot, would have successfully introduced clicks into Zulu. Thus those older writers who postulated "mixed languages" as a result of "racial" mixture may have been closer to the mark than intervening generations have judged them to be, though of course for the wrong reasons.

${ }^{14}$ In the first approximation to a theory, we pass over in silence the complex set of circumstances in which the sum of morphemic regularity, rank differential, and upward mobility is equal to zero. 
13.3. Once all variables are above zero, their effects, relative to each other, probably change rapidly. For example, phonemic regularity, crucial if it is zero, probably does not add much as it approaches 1. The frequencies of occurrence and interaction probably have the greatest effect followed by rank differential ${ }^{15}$ and upward mobility.

These hunches are partly reflected in the measures chosen for the divers variables, partly left to the eventual corrective action of the values to be assigned the constants.

14. Testing for Innovation

14.1. Of course any spontaneous use of the test word-in a context where it is not a quotation-may be regarded as a test of the theory. There remains the problem of what to do if a month or so of dogging the informant's footsteps has produced no results one way or the other.

14.2. In such circumstances it is advisable to conduct an interview in which the conversation is steered towards a context in which the test word or a competing word is probable. It is of course important that the test word not be used by the investigator. ${ }^{16}$

15. Some Actual Predictive Theorems

15.1. Other factors equal, a tory $y$ will adopt the phonemically more regular of two test words more rapidly than he will adopt the phonemically less regular one.

15.2. Other factors equal, a tory $y$ will adopt a test word supplied by a model $x$ more rapidly than he will adopt one from a model $w$ if the social rank of $x$ is higher than that of $w$.

15.3. Other factors equal, a tory $y$ will adopt a test word more rapidly than will a tory $z$, if the upward mobility of $y$ is greater than that of $z$.

15.4. Other factors equal, a tory $y$ will adopt the most frequently

${ }_{15} \mathrm{G}$. P. Murdock has suggested that the effect of rank differences probably decreases with distance of the model's rank from that of the tory as a result of the empirical finding that respondents often have not learned the cues that distinguish remote classes from each other, i.e., a lower-lower respondent may lump together both upper-middle and lower-upper respondents as "big shots." Thus the curve of rank effect is probably negatively accelerated.

${ }^{16} \mathrm{R}$. D. Schwartz has suggested that the likelihood of the informant's using the word is highly dependent on whether he thinks the listener will understand it. This poses problems for ascertaining the effect of the investigator as listener in the test interview. 
presented of two test words more rapidly than he will adopt the less frequently presented one.

16. Conclusion

Many more theorems like those of the above sample can be generated. Such predictive theorems are the most satisfactory way of ascertaining the effects of the variables, their mutual relations and dependencies, and thus, testing the theory. The theory pertains to but a small part of the relations between linguistic and other cultural systems; if it proves to be supported by the evidence, the results should be two-fold: vindication of those pioneers who dared commit themselves with respect to some of the non-linguistic correlates of linguistic change, and the generation of associated formulations concerning similar ethnolinguistic problems such as bilingualism and linguistic acculturation.

\section{Literacy as a Problem in Language and Culture}

\section{PAUL L. GARVIN}

\section{Institute of Languages and Linguistics, Georgetown University}

I would like to present a case study in language and culture.

On my field trip in 1947 to Ponape, an island of the Eastern Carolines in Micronesia, ${ }^{1} \mathrm{I}$ had, in addition to the usual linguistic research, the practical objective of devising a unified spelling system for the island. I would like to discuss some of the linguistic and cultural problems of interest that emerged in the course of this enterprise, of the practical success of which, incidentally, I am by no means assured.

There was no question of the need for a major spelling reform on Ponape: for a speech community of about 5700 , most of the adult and adolescent members of which are at least partially literate, I counted

1 The field trip was undertaken as part of the author's participation in the Coordinated Investigation of Micronesian Anthropology, under the sponsorship of the Pacific Science Board of the National Research Council, June-December 1947. In addition to the sponsoring agency, many agencies of the U. S. Navy and other branches of the U. S. Government extended their support to make this research project possible, all of which is bereby gratefully acknowledged. 
after my arrival at least five different spelling systems in varying degrees of use and disuse. The reasons for this proliferation of orthographies are to be found in the unusual acculturative history of the island: after the first contact with Yankee whalers in the 1820's, no fewer than four major Western or Westernized nations have at one time or another drawn Ponape into their political and cultural sphere of influence, with the attendant influx of missionaries, administrators, seafaring and armed forces personnel, and-within limits-even colonists. In the 1840 's, the Boston Mission inaugurated an extensive program on the island, in the 1870's Spain acquired it aspart of its Oceanic colonies and sold it to Germany after the Spanish-American War. Early in the First World War, Japan occupied the island and kept it after the Versailles Treaty as a League of Nations mandate. The end of the Second World War saw, first Military Government and Civil Administration by the U. S. Navy, and later American civilian administration as part of the Trust Territory of the Pacific Islands. Protestant Missions have been active on the island since the 1840's, Catholic Missions since the Spanish rule. The population is at the present about evenly divided between Catholics and Protestants. With the changes in political administration, mission groups of different nationalities succeeded each other, although the religious affiliations of the islanders remained largely unaltered. Each of the various missions published its own literature in Ponapean and, most unfortunately, used its own orthographic inventions, creating various degrees of language loyalty to them. Hence the minor graphemic Tower of Babel which I encountered. Needless to say that none of the systems in use, either singly or-as was frequently the case-in combination, has been particularly adequate for purposes of functional literacy.

In order to do more than merely increase the existing confusion by the addition of one more rival orthography, I thus had not only to devise an adequate and generally agreeable system, but also to attempt to bring about its acceptance by the Ponapeans and others concerned.

In the third month of my stay on Ponape, when I had become sufficiently familiar with the language and the problems involved in the promulgation of a new orthography, I formulated a few of the basic 
ideas for the proposed spelling reform. I discussed these ideas with several prominent Ponapeans in various parts of the island, and with interested local whites. Since the reaction to my ideas was generally favorable, I proposed a meeting of the teachers, native preachers, and higher chiefs of the five major political subdivisions-called districts -of the island, at which to present my suggestions to the larger audience. This meeting took place shortly afterwards, and in addition to the named Ponapeans was also attended by representatives of the U. S. Navy Civil Administration Unit, a local missionary, and an interested white settler. I addressed the meeting in halting Ponapean, and it was decided that I should proceed with the spelling project, aided by a commission of five Ponapeans, one from each of the districts. The Commission consisted of one district chief, one native preacher, and three schoolteachers. The former two had served as my informants for quite some time previously. They were both fairly educated men, fluent speakers of German, and able to read German books and use German dictionaries. In addition, their informant work with me had given them considerable insight into the structure of their language. I met with my Ponapean co-workers almost daily over a period of more than a month, during which time we ironed out the details of the new orthography and also prepared the text, in Ponapean, of a Ponapean spelling book to be used in promulgating the new system.

The problems to be solved fell into the following categories, in approximately decreasing order of importance.

(1) The selection of the linguistic pattern to be represented by the spelling system-in short, of a Ponapean standard language;

(2) Deciding on the most suitable orthographic representation of the standard language;

(3) Orthographic solutions of morphophonemic and morphemic problems such as word boundaries, variability in the phonemic shape of morphemes, and the spelling of multiword lexical units;

(4) The spelling of loan words;

(5) Secondary orthographic details: points of syllable division, use of capital letters, punctuation;

(6) Problems of style and terminology contingent upon the preparation of the Ponapean spelling book. 
In dealing with each of these problem areas I had, to paraphrase Pike, the benefit of sophisticated native speaker's reaction:2 I presented and explained possible alternative solutions of the various problems to the Ponapean commission, and in deciding between solutions, the preferences of the Commission members were consistently taken into account.

Let me now discuss the six problem areas in some detail.

1. Selection of a standard language. Ponapean has two major dialect divisions: the Kiti dialect, spoken in the district of the same name, and the Main dialect, spoken in the remaining four districts.

The two dialects agree in their consonantal pattern: they both have $p, t, t$ (retroflex), $k, m, n, b, s, r, I$; there are no consonant differences in dialect cognates. The same applies to the semivowels $w$ and $y$.

The dialects differ in their vowel inventory: the Main dialect has eight vowels-a, $0, \varepsilon, o, e, u, i, \ni$; the Kiti dialect has seven vowels$a, o, o, e, u, i, a$ (in the Kiti dialect, $[\varepsilon]$ and [e] are positional variants of $/ e /)$.

In both dialects, vowels are in addition differentiated by the prosodic feature of quantity. Stress is phonemic.

The most prominent phonological difference between the dialects lies, however, not in the vowel inventory but in the distribution of vowels in cognate morphemes. A sizeable number of morphemes which contain / $a /$ in the Kiti dialect, have either $/ \varepsilon /, / e /$, or /o/ in their Main dialect cognates; a lesser number of Kiti morphemes containing / / have / $/$ / in their Main dialect cognates; and in a few cases Kiti has /a/ where Main has / $/$.

Neither dialect community is willing to accept the preëminence of the other; hence, selection of either the Kiti dialect or the Main dialect for the standard underlying the new orthography would have provoked the antagonism of the speakers of the other dialect.

I therefore proposed that the reformed spelling represent a sort of "overall pattern," based on a compromise between the two dialects. Although this suggestion violated the one-by-one correspondence of phonemes to graphemes for either dialect and therefore was apt to increase the learning difficulty, it was enthusiastically received by

" Kenneth L. Pike used the term "naive native speaker's reaction" consistently during the 1951 session of the Summer Institute of Linguistics at the University of Oklahoma, Norman, Oklahoma. 
both Kiti and Main dialect speakers, as the only way in which acceptance by both dialect communities could be assured.

This overall graphemic pattern was to be so constructed that, with very few unavoidable ambiguities, the graphemes could be read off with the phonemes of either dialect with equal ease.

No problem existed, of course, for the consonants and semivowels, since they are shared by both dialects and distributed equally in all cognates: one grapheme had to be assigned to each consonant and semivowel phoneme.

In the case of the graphemic representation of the vowels, two different sets of situations had to be faced: cases in which the cognates of the two dialects have the same vowel phonemes, and cases in which the vowels in the cognates differ.

The complication introduced by the difference in the vowel phoneme inventories of the two dialects (two mid front vowels in the Main dialect versus a single mid front vowel in Kiti) turned out to be apparent only. All those cognates which in the Main dialect are minimally differentiated by the opposition of open versus closed mid front vowel, are in the Kiti dialect differentiated by a corresponding opposition of the low vowel /a/ versus the single mid front vowel /e/: thus, Main dialect has /séscy/ rowing versus /seysey/ haircut; to this correspond Kiti dialect / sáysay/ and /séysey/. In other cases, where the opposition of openness for mid front vowels in the Main dialect is not minimally differentiative, it could be ignored in the graphemic pattern without reducing the unambiguous intellegibility of written texts by speakers of either dialect.

In summary, the following specific cross-dialectal vowel correspondences exist:

(1) Cases in which vowels are the same, or nearly the same (i.e., Main dialect $/ \varepsilon /$ or $/ \mathrm{e} /=\mathrm{Kiti} / \mathrm{e} /$ ):

$$
\begin{aligned}
\text { Main } / \mathrm{a} / & =\mathrm{Kiti} / \mathrm{a} / \\
\text { Main } / \mathrm{o} / & =\mathrm{Kiti} / \mathrm{o} / \\
\text { Main } / \mathrm{o} / & =\mathrm{Kiti} / \mathrm{o} / \\
\text { Main } / \varepsilon / \text { or } / \mathrm{e} / & =\mathrm{Kiti} / \mathrm{e} / \\
\text { Main } / \mathrm{u} / & =\mathrm{Kiti} / \mathrm{u} / \\
\text { Main } / \mathrm{i} / & =\mathrm{Kiti} / \mathrm{i} / \\
\text { Main } / \mathrm{a} / & =\mathrm{Kiti} / \mathrm{a} /
\end{aligned}
$$

Each of these cases could be adequately represented by a single grapheme, necessitating in all 7 vowel graphemes. 
(2) Cases in which vowels differ:

$$
\begin{aligned}
\text { Main } / \varepsilon / \text { or } / \mathrm{e} / \text { or } / \mathrm{a} / & =\mathrm{Kiti} / \mathrm{a} / \\
\text { Main } / \mathrm{a} / & =\mathrm{Kiti} / \mathrm{o} / \\
\text { Main } / \mathrm{o} / & =\mathrm{Kiti} / \mathrm{a} /
\end{aligned}
$$

Ideally, these three cases would require 5 additional graphemes to cover every situation unequivocally for both dialects. In practice, however, it was found sufficient to posit a single additional grapheme to cover all the cases, and they are many, in which either $/ \varepsilon /$ or $/ e /$ or $/ \partial /$ in the Main dialect corresponds to /a/ in the Kiti dialect. In the relatively few cases where Main /ə/ corresponds to Kiti /o/, and where Main /o/ corresponds to Kiti /a/, another solution was proposed: to use, from among the 7 graphemes with unambiguous phonemic value, alternatingly either that representing the value of the vowel in the Main dialect cognate, or that representing the vowel in the Kiti cognate.

Irrespective of the graphemic alternative chosen, all the above cases in which vowels differ in the two dialects were to be listed in an orthographic glossary, since their correct spelling could not be predicted from the phonemics of either dialect alone, although if seen in writing, they could be recognized unambiguously by both Main and Kiti speakers.

Of the two prosodic features, quantity and stress, only the former has sufficient functional yield to require graphemic representation. Stress could be ignored in the orthography. The difference in functional yield is based on the following criteria: greater stability of quantity (some cases of alternative stress were found, but none of alternative quantity); easy availability of minimum pairs for quantity - frequently volunteered by native speakers-versus none for stress.

In all then, ten consonant graphemes, two semivowel graphemes, eight vowel graphemes, and a graphemic representation of quantity had to be accounted for by the new spelling system.

2. Choice of orthographic symbolization. The grapheme inventory of the proposed standard Ponapean thus, as outlined above, turned out to consist of twenty graphemes and the vowel feature of quantity, in all twenty-one units. Since this is less than the number of letters in the Roman alphabet, theoretically, each grapheme and the feature of quantity could be represented by one of the letters, with letters to spare. This would, however, have required assigning completely 
arbitrary values to some of the letters, which would have created considerable confusion, in view of existing spelling habits and foreignlanguage literacy, especially in German and English. Those graphemes which correspond to phonemic values different from the "usual" phonetic values of the Latin letters, as well as the feature of quantity, thus had to be rendered by some other means. The "problem" graphemes included four having the cross-dialectally unambiguous phonemic values of $/ \mathrm{t} /, / \mathrm{g} /, / \mathrm{o} /$, and $/ \mathrm{a} /$, respectively and one having the variable cross-dialectal phonemic value of $/ \varepsilon /$ or $/ e /$ or $/ \partial /$ in the Main dialect, $/ a /$ in the Kiti dialect. I shall call the latter the "variable" grapheme.

I proposed two alternative orthographic treatments of these five graphemes and of the feature of quantity to the Ponapean commission, which for the sake of convenience I shall label the "Finnish" and the "German" solution respectively.

Both solutions agreed in the treatment of the grapheme for $/ \mathfrak{y} /$ : it was to be represented by the digraph "ng". For the remaining "problem" graphemes, the Finnish solution used diacritics, the German solution digraphs, in the following manner:

\begin{tabular}{|c|c|c|}
\hline Grapheme for: & "Finnish" solution: & "German" solution: \\
\hline$/ \mathrm{t} /$ & "ț" & "t" (as opposed to " $d$ " for $/ t /$ ) \\
\hline /al & "g" & "oz" \\
\hline$/ \partial /$ & 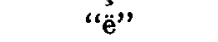 & "oe" \\
\hline Variable grapheme & “ä" & "ae" \\
\hline
\end{tabular}

The "Finnish" solution for indicating phonemic quantity was the doubling of long vowels, as follows: "aa", "ää", etc.; the "German" solution was to use " $h$ " as a length mark, as follows: "ah", "oah", etc.

The commission members found the "German" solution awkward; they objected especially to the trigraphs necessary to render long vowels which, when short, are represented by digraphs, such as "aeh", and necessitating no less than tetragraphs for long diphthongs, such as "aehy".

It was therefore decided to adopt the "Finnish" solution and proceed with other details of the orthography. The two diacritics needed, the cédille and dieresis, could easily be produced on a typewriter by backspacing to make a comma under the letter or a quotation mark 
over the letter. Both diacritics were already known to the natives from previous spelling systems.

The alphabet used for Ponapean in accord with the "Finnish" solution thus included the following letters, with one digraph: a, a, e, ë, i, k, l, m, n, ng, o, $,, p, r, s, t, t, u, w, y$. The remaining Roman letters were available for use in spelling unassimilated loans.

3. Morphophonemic and morphemic problems. Agreement upon a suitable set of alphabetic symbols by no means exhausts the range of orthographic problems. The next decision to be made related to the matter of word boundaries-in orthographic terms, the matter of spaces to be left between sequences of letters.

The linguistic definition of the Ponapean word as a sequence of morphemes and morpheme clusters in fixed relative order, is not completely adequate for orthographic purposes, because it conflicts in part with the native speaker's use of the word in the sense of Voegelin's operational definition: the word is the smallest unit volunteered in isolation by a native informant. ${ }^{3}$ In fact, the Ponapean commission agreed to the linguistic word boundaries I proposed for verbs, but not for nouns. In the case of the latter, they preferred to write incorporated expressions and phrases followed by noun suffixes with spaces between each word of the expression or phrase and each cluster of suffixes. As a result, orthographic words did not in every case coincide with morphemic words.

Still another discrepancy between the linguistic pattern and the new orthography arose in the realm of morphophonemics, again as a result of the cultural desideratum of acceptability overriding purely linguistic considerations. Linguistically, the framework for all morphophonemic processes leading to morphophonemic variation and morpheme alternation, is the contour, which is a unit bounded by pauses and characterized by a single primary stress. Any contour may contain one or more (or even less than one) linguistic words, and, mutatis mutandis, one or more orthographic words. Nonetheless, morphophonemics was accounted for orthographically only within orthographic words, not across orthographic word boundaries, as follows: phonemic /totónki/ work with would yield a single orthographic word, with the morphophonemic dissimilation of the first of

s C. F. Voegelin, Multiple Stage Translation, address to the Washington Linguistic Club on 8 April 1954. Paraphrased by me from memory. 
two contiguous stops rendered by the spelling, thusly: "totogongki". Phonemic /toto' $\eta^{n} k \varepsilon \cdot l a y l / 4$ hard work would yield two orthographic words, with the morphophonemic dissimilation ignored by the spelling, thusly: "totogk keelayl". This inconsistency was introduced into the spelling system because of the strong feeling of the Ponapean commission for the need for keeping the orthographic shape of individual words constant. Perhaps this preference reflects the linguistic fact that in Ponapean each full word may potentially constitute a complete and separate contour.

One exception to this disregard of morphophonemic processes at word boundary was adopted into the spelling: wherever the initial vowel of certain particles is elided after a full word, this was to be reflected orthographically and indicated by the use of an apostrophe. Thus, phonemic / wèy^ $n^{\wedge} n \varepsilon t$ / district of Net, showing elision of the vowel of the hypotactic particle, would yield orthographic "wey'n Net". The commission members felt that some solution was required for these cases, since elison had in some ways been accounted for by previous spelling systems, though not satisfactorily. When I explained the analogous use of the apostrophe in French to them, they agreed to accept it for Ponapean. I assume that some prestige feelings may have had a part in their decision.

An additional inconsistency from the standpoint of morphemic word boundaries was introduced in the case of lexical units consisting of more than one word, which in Ponapean are usually constituted by hypotactic noun expressions. These were in the orthography linked by hyphens, and if the vowel of the hypotactic particle was elided, the hyphen was replaced by an apostrophe as indicated above, as follows: phonemic / $\mathrm{ol}^{\wedge} \varepsilon \mathrm{n}^{\wedge}$ wáy/ foreigner would yield orthographic "ol-en-way"; phonemic /wàsa`^n^ tóto•k/ place of work with elided / $\varepsilon /$ of the hypotactic particle, would yield orthographic "wasaa'n-tọtogk".

4. Loanwords. The Commission agreed to my suggestion that assimilated loans should be spelled in the native manner, in accord with the rules laid down in the previous sections; unassimilated loans (in practice, most of the English loans introduced since the American administration took over from the Japanese) should retain the spell: ing they have in the original language. The Commission itself was to

4 Superseript arcs in the phonemic transcription indicate word boundaries within contours. Forms are cited in the Main dialect. 
decide which of the loans would fall in either of the two categories. Among assimilated loans, they placed such items as "nayp" knife, "sitoosa" automobile, "sirangk" wardrobe; among unassimilated loans, such items as movies (phonemically:/múpis/), jeep ( $=/ \mathrm{si} \cdot \mathrm{p} /)$, business ( $=/$ pisnis/), although each of these is in fact phonemically assimilated to Ponapean, and is in quite common use. Nonetheless, their "Sprachgefühl" placed these and other similar words in the unassimilated category.

5. Points of syllable division, capital letters, punctuation. These orthographic devices were in some way or another utilized by all previous spelling systems, and the Commission felt they should be retained in the new spelling, but that their use should be standardized by a set of definite rules.

In regard to points of syllable division, all agreed that a single consonant or semivowel should be counted towards the following syllable; in clusters of two consonants (larger clusters do not occur in Ponapean), the point of syllable division should fall between the two consonants; in cases of consonant and adjacent semivowel, the point of syllable division should fall before the consonant even where the semivowel follows the consonant.

Initial capital letters were decided upon for the first words of sentences or headings, personal names and place names, all chiefly titles, titles of foreign officials and agencies (most of these would fall into the category of unassimilated loans discussed in the previous section), religious titles and holidays (including, of course, the name of the Deity), all honorific pronouns. An interesting discussion developed among the commission members regarding the use of capital letters for chiefly titles: one member of the commission felt that some of the lower titles did not deserve the honor of capital letters, but the others convinced him that such discrimination would create bad feeling among those concerned, and so the rule was formulated specifically to include all chiefly titles. Strangely enough, no need was felt to capitalize the Ponapean equivalent of "Sir" which is most often used as an honorific term of address to foreigners.

Sentence-final punctuation presented no problem. In the matter of punctuation within the sentence, I explained to the Commission the difference between the West European tradition, as exemplified by English, of punctuating approximately at phonological breaking 
points and logical boundaries on the one hand, and on the other hand the Central European tradition, as exemplified by German, of punctuating approximately at syntactic boundaries. The Commission members showed a definite preference for the West European procedure, and the rules were formulated accordingly.

6. Preparation of the spelling book. This was the most important phase of my work relating to Ponapean orthography. The spelling book was to serve as the vehicle for the introduction of the new system; also, it was during the preparation of the spelling book that most of the orthographic details were worked out.

The working procedure was as follows: the contents of each section of the spelling book were first discussed in detail with the members of the Commission, on the basis of my recommendations. Upon reaching agreement, I would attempt to formulate, sentence by sentence, the pertinent passages of the book in my own imperfect Ponapean, which would then be corrected and improved by the Commission. Next, I would write each sentence separately on a blackboard that we had available, and final corrections were made by Commission members. Upon definitive acceptance, the sentence would be copied for inclusion in the spelling book.

Rather than being in the customary ethnological rôle of participant observer, $I$ thus found myself in the position of a sort of observant participant in the culture. My deficiencies in Ponapean were balanced by my experience in the organization and formulation of written matter, and between the Commission and myself, we worked out a Ponapean style suitable for use in highly literate writing of this nature. All Commission members shared, and expressed, a feeling of pride in the ability of their language to express such relatively complex material adequately; all of them had also in the past been skeptical as to the possibility of doing so. Our success was, of course, no more than a confirmation of Sapir's well-known statement that "language is a perfect symbolism of experience." Ponapean non-verbal culture had already, through acculturation, acquired a good many urban features, ${ }^{6}$ and the language was on its way to catch up with it. I felt

${ }^{5}$ Edward Sapir, Language, reprinted from Encyclopedia of Social Sciences in David Mandelbaum, ed., Selected Writings of Edward Sapir in Language, Culture, and Personality, Berkeley, 1949, p. 12.

- These terms are here intended in the sense of Robert Redfield's folk-urban scale. 
that it was my function to assist in this linguistic urbanization of a folk community, as an aid in its adjustment to an urban environment, and, if I may inject a personal note, I was gradually led to share my Ponapean friends' pride in their verbal achievement.

While my observations on style must, at this level of my analysis of Ponapean, remain impressionistic, I should like to present, as my last point of detail, another index of linguistic urbanization which I am in a position to discuss more specifically. I am referring to the technical terminology used in the spelling book.

Here again, whatever innovations the Commission and I made were merely an extension of existing practice since a number of the terms needed in the formulation of the spelling rules were already in use in Ponape. In adding to these terms, I had an opportunity to test the potentialities of the Ponapean language for adjustments to new cultural needs by the adoption of viable lexical units to meet these needs. By viable, I mean lexical units which both phonemically and morphemically are easily absorbed into the pattern; on the basis of my vicarious "Sprachgefühl" I would impressionistically call them easy to handle, "modern", or even "streamlined".

The mechanism of vocabulary expansion in Ponapean includes the utilization of existing productive categories for the formation of new native units, the phonemic assimilation of loans from three foreign languages-English, German, Japanese-and a combination of both processes. The existing spelling terminology represented a cross section of all three of these procedures; additions to it could therefore likewise draw upon these resources of the language.

The operational procedure followed in the creation of new terminology was similar to that followed in other phases of the work. Whenever the need for a new term arose, I would explain its content to the Commission and ask for suggestions. In many instances, native formations were immediately volunteered and agreed upon. In those cases where the Commission members were unable to produce a terminological solution, I would attempt to create a term myself by analogy to existing native formations. If I was unable to do so, or if my suggestion was deemed unsuitable by my Ponapean co-workers, I would then suggest both the German and the English term for possible adoption as a loan, and ask for their preference. Upon reach- 
ing final agreement, the term would be included as an assimilated loan, that is, using Ponapean orthographic symbolization.

Let me give a few examples.

Names had to be given to the letters " $w$ " and " $y$ ", not previously used in Ponapean spelling. Consonant letters in use so far were consistently named by adding the vowel $/ \mathrm{i} /$ to the consonant, thus /pi / for "p", /si\%/ for "s", etc. Hence, the semivowels were by analogy named /wi / and /yi / respectively, orthographically "wii" and "yii".

A name was needed for the cédille. No native name was proposed, so I suggested the inclusion of "cédille" as a loan, which became phonemically /setil/, orthographically "setil"-a normal phonemic shape for a Ponapean morpheme.

A term was needed to denote "parenthesis". Again, no native suggestion was forthcoming. I asked for the Japanese term which my co-workers did not know. Then I proposed a choice between English "parenthesis" which could be loaned into Ponapean as */paréntesis/, and German "Klammer" which could be loaned into Ponapean as /klámmar/. The Commission expressed a strong preference for the latter term because of its more normal phonemic shape; it was adopted as orthographic "klammer".

Terms were needed for punctuation marks; the existing term for

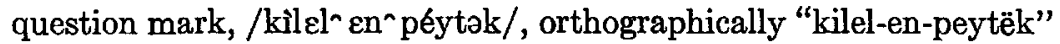
(literally: "sign of question"), provided the pattern for a number of

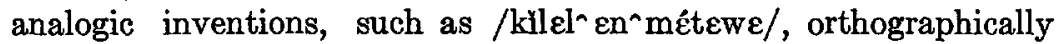
"kilel-en-mätäwä" dash (literally: "sign of thought", based on German "Gedankenstrich").

The problem of devising an acceptable spelling system, which initially might have appeared purely, or at least primarily, a linguistic matter, upon closer inspection thus turned out to be a language and culture problem par excellence. At each step, linguistic judgement had to be tempered by the consideration of cultural attitudes, traditions, and even prejudices, as the closely intertwined patterning of verbal and non-verbal behavior unfolded before the eyes of the observer. 


\section{Non-segmental Elements in Foreign Language Learning}

\section{WILLIAM E. WELMERS}

\section{Cornell University}

You may be wondering just what connection there is between my subject and the general topic of this panel: Language and Culture, language in its broad cultural setting. Well, I have been wondering the same thing myself. I am quite sure, however, that there is a connection, and that none of us realizes just how deep and intimate that connection is. I am going to speak largely in terms of language structure and techniques of language teaching, but I hope that I will be able to convey some impression of the implications of a linguistic problem in an area wider than that of simple linguistic communication.

By the term 'non-segmental elements', I mean features of pitch, intensity, and duration in language, as opposed to consonantal and vocalic phonemes. I hold no brief for the terms 'non-segmental' and 'segmental', but I believe that there are valid reasons for setting up a dichotomy between the types of phenomena indicated by these terms.

From the point of view of the history of language teaching, it is certainly these non-segmental elements that have received the least attention. A few passing remarks on 'accent' are about the most that can be expected from the average language textbook. A commercial recording purporting to teach something about the pronunciation of Classical Greek illustrates the difference between short and long vowels by comparing a vowel sound about one-tenth of a second long with one that, as I recall, must have lasted at least two seconds. The complete absence of realism defeats the purpose of the comparison. For some of the more exotic languages of the world, grammarians still observe with alarming frequency that such things as tone and stress 'can only be learned by observation and practice'-a statement that Bloomfield characterized as 'nothing less than a downright swindle', since observation and practice are the only way to learn anything about a language. It is a slight improvement to learn from some books that in the languages they describe 'tone is very important'. But in a vast number of cases this remark is followed by three or four examples of minimal contrasts in tone, concluding with the statement: 'In this book, however, tone will not be indicated'. It has only been 
within the last few years that systematic efforts have been made to analyze and teach intonation.

It is perhaps a cause and perhaps a result of these factors that nonsegmental elements in language are often assumed to be something outside the linguistic system-something characteristic of the human race as a whole, or at best something associated with a given culture. It is not without reason that many Americans believe the British to be snobs, that many Britishers believe the Americans to be crude, and that many speakers of English believe the Japanese to be unusually precise. A common intonation for questions in British English is used in this country almost exclusively in speaking to children or in circumstances of personal intimacy. When the Britisher says 'Are you going to town?', it makes the American feel like a child or like someone who needs special tender handling; therefore the Britisher is a snob. Conversely, the American intonation with the same question strikes the Britisher as impatient, rude, and blunt. Therefore the American is all of these things. The Japanese speaks English with syllable-timed rhythm, and perhaps uses vowels of equal length before voiceless and voiced consonants. There is something mathematical about the precision of it, and therefore the Japanese is precise and mathematical.

It is no wonder, then, that non-segmental elements in language are especially difficult for students to master. Students must first be persuaded that such elements exist! Once that is realized, some of the difficulties may be primarily in the students' imagination, but they exist none the less. The very mention of 'tone' is enough to frighten a good many would-be linguists, and even experienced language analysts and teachers claim that they 'cannot hear tone'. Since I have worked on a number of tone languages, I enjoy the reputation in some circles of being a minor genius. The reputation is undeserved, but it does no harm to my ego, and reflects the aura of mystery and profundity that surrounds problems of non-segmental linguistic features.

I suspect, however, that there is a more basic reason why these problems differ from problems of learning and teaching consonantal and vocalic sounds. Pitch, intensity, and duration are significant characteristics in the entire realm of sound, not merely in language. A note played on a violin may be higher or lower in pitch, louder or softer, longer or shorter. Distinctions of pitch in a dog's whine, of intensity 
in the footfalls in the apartment upstairs, of duration in the horn of the car just behind you - all these are calculated to stir us into action. But-with the possible exception of a few trick imitations like that of a muted trumpet-consonants and vowels are peculiar to human speech. It is obvious to the learner of a new language that consonants and vowels are going to be of functional importance in that language. It is not so obvious that pitch, intensity, and duration are going to be of functional importance in a language. We all recognize such distinctions in our daily life, but it is not immediately obvious that they may have a specifically linguistic function, or just what that function is or may be. Incidentally, the function of pitch, intensity, and duration in the realm of sound outside language as well as within it-as opposed to the uniquely linguistic function of consonantal and vocalic articulation-has suggested to me the possibility of calling these features 'para-linguistic'. Our problem concerns the linguistic function of these 'para-linguistic' features.

In learning a foreign language, the student's tendency to transfer habits from his native language to the language he is learning applies to both segmental and non-segmental features-or, if you prefer, to both uniquely linguistic and para-linguistic features. This is not even to mention the problems of transfer in grammar and lexicon. In the case of consonants and vowels, however, even a considerable amount of transfer is likely to result in little more than somewhat laborious communication, with an occasional embarrassing or hilarious confusion such as that experienced by the missionary who for a year thought he was saying 'Let us bow our heads' only to learn that, because of using the wrong vowel in one word, he had been exhorting the people to vomit. In the case of non-segmental elements, on the other hand, transfer of native habits is likely to extend far beyond these limits. There is certainly enough of overall difficulty in communication. Another missionary discovered that the audience to which he had preached didn't even realize he was attempting to speak their language-in this case, a difficulty probably largely attributable to his complete disregard for the tonal structure of their language. There is no lack of isolated confusions either. Every discussion of this type should include at least one prize example. My favorite is not particularly funny, but is at least striking. In the More language of French West Africa, the words for 'God' and 'sin' differ in tone, in the length 
of a vowel, and in the presence vs. absence of nasalization. The words are distinguished in writing also, but the spelling difference has no relation to any of the three phonemic differences between the words!

Beyond this point, however, the transfer of native habits in nonsegmental elements is likely to involve all sorts of additional troubles. If a language distinguishes verbal aspects by tone or stress, the learner may unwittingly miss a large area of systematic grammatical distinctions in his hearing and in his speaking. The contrast between negation and exhortation in Fante is tonal. Pluralization is often tonal in Tigum. And misunderstandings are inevitable in the subtleties of emphasis, question-asking, surprise, and the whole range of connotative overtones. A learner of Kpelle was able to say 'one cent' 'quite understandably, but when she tried to say 'ONE cent (not TWO)', her utterance was meaningless, because there is no word segmentally like the word for 'cent' with the tone she used in an effort to emphasize 'one'. Very few Americans learning Kpelle have ever succeeded in asking questions gracefully. The Kpelle question intonation sounds like a surprised or rude question in English, and the American-unwilling to betray surprise or to act rude - succeeds mainly in making a fool of himself. It is here that the implications affecting culture and cross-cultural relations lie. The problem has barely been touched, but perhaps we are at least stating it. I'm not sure how the Kpelle people feel about the missionary who pronounces the benediction in a service otherwise conducted entirely by native speakers of Kpelle. My reactions are by no means native, but it just may be significant that, when I heard it all recorded on tape, I got the impression that the missionary didn't really care a bit if the Lord blessed the people or not. Is it possible that at least a bit of the antagonism and unrest blamed on 'imperialism' should be attributed to an ethnocentricity evidenced by the miserable failure of explorers, missionaries, anthropologists, businessmen, and colonial officers to recognize and imitate tone, intonation, stress, and length? (By the way, missionaries have often borne the brunt of smug criticism in this respect. It has been my observation that, on the whole, they are the least guilty of all the groups I have mentioned.) At the very least, the learning of non-segmental features of a new language seems to be a special problem, and perhaps it requires a special solution.

In the preparation of teaching materials for English and a number 
of other languages, and particularly in teaching Americans to speak tone languages, I have had occasion to experiment in a limited way with techniques and types of drills. Having painted the background at some length, $J$ can outline these central figures in my picture quite briefly.

The very nature of the problem would seem to require that it be handled from the very beginning of the student's attempts to learn a new language. Contrasts in pitch and intensity and duration are not mere details that can be filled in later, but are basic problems that may involve the worst kind of misunderstandings. And if we start at the beginning, we have an opportunity to do what I believe will prove most valuable: require the student to imitate without knowing the meaning of what he says! I think you can all hear a difference between these two utterances: / a làli pala/ and /a lâli pàla/. If I were to tell you the meaning of these utterances, you-if you are a normal student -would concentrate on remembering the meanings at the expense of mastering the contrast, and in addition you might become confused and discouraged. But I am not going to tell you anything about the meaning. As far as you know, I may have been talking about two utterly different things. Or I may first have asked a question and then made a statement. Or perhaps I said 'the same thing' with two different emphases. Or possibly I was referring to the same action taking place at two different times. You have no idea what distinction I was making. As long as that is true, you will try to imitate accuratelyperhaps because you can only imagine what horrible consequences might ensue if you made a mistake. I can instill a habit of recognition and imitation in this way, unencumbered by your smug awareness that I am not going to tickle the soles of your feet if you say the wrong thing. And if you were to hear me say /à làli pala/ three times and /a lâli pàla/ three times, I would almost guarantee that you would remember the distinction long enough to ask me afterwards what the two utterances mean. That is learning a language!

From the beginning of the learning process, the student can also use a type of drill which may well come to be known as 'the monotony drill'. Such a drill has two or more parts. Each part is a group of short utterances identical in their non-segmental features, but differing in segmental phonemes. For drill on English stress, such 
a group might consist of the forms teacup, meat ball, door knob, pie tin, soup spoon, tie clasp, necktie, hide-out. Other groups illustrate contrasting patterns, like three times, hide here, tie knots, and so on. What the student may miss in a single utterance, he is almost bound to hear in a monotonous repetition, particularly if he is told just what detail to listen for. Meaning is not particularly relevant, but may be gradually introduced, particularly for very common forms.

Having established the recognition of several different patterns, the student can begin problems of comparison. Into which group does a new utterance fit? Such patterns can, in turn, be treated as the constituents of longer utterances. In time, all of the endless variety of combinations of non-segmental patterns can be reduced to the limited number of elements that enter into them. We want our students to imitate like children, but perhaps we have failed to expose them sufficiently to the endless repetition of simple patterns that is part of the child's experience.

Finally, a judicious use can be made of the very transfer tendencies we are trying to avoid. In imitating an informant, a speaker of English will very frequently repeat what he has heard, but with an English question intonation that means 'Am I saying it right?' If he is learning a tone language, and if the sentence ends with a low or falling tone, the imitation is automatically NOT right. I have had to tell students to talk as if they are sure of themselves, to TELL the informant what he said, and even to get mad and yell it at him. Eventually, something works. For sentences that end with a mid tone, Americans often imitate more accurately if they are told to say such sentences hesitantly. If the student learns that every sentence that ends with the word for 'there' sounds hesitant, he will eventually divorce the nonsegmental pattern from the English intonational morpheme meaning 'hesitation', and associate it with a word meaning 'there'. But meanwhile, he is speaking accurately, and that is the important thing.

All of what I am trying to say boils down to something very simple, but something which has often been ignored. The teaching and learning of non-segmental linguistic elements is most important-perhaps far more important than we have yet begun to realize. It is also subject to systematic treatment. And it is by no means as hopeless a task as we may have thought. 


\section{Discussion}

In the discussion after the third session the following points were brought up:

HALLE (MIT): I would like to address my question to Professor Garvin-what kind of alphabetic order did you impose? You see the question of alphabetic order is quite important because it is one of the most important ways in which information is arranged in our language. What did you do about the extra letters?

GARVIN: I put the letters with diacritics after the letters as they would come in the ordinary Roman alphabetic sequence, simply because this has been customary before.

A. A. HILL: I should like to point out that I was extremely interested in Professor Garvin's paper, since all too frequently the assumption in devising a system of writing is that probably the real purpose of the system of writing is to make the linguistic structure of the native language available at a glance to any wandering linguistic analyst. In this instance, it seems as if the approach has been very different. It has been not merely one of attempting to satisfy all cultural demands and prejudices and feelings, but also the necessary pragmatic approach of a system of writing which is that, essentially, a system must perform one of two aims. It doesn't necessarily have to perform both, but if it performs neither, then it is a failure. One aim is that a native speaker of the language should be able to look at the forms and recognize them as forms in his native speech. In other words, the predictability from the symbol. The other is that the native speaker should be able to say something and predict what the symbols are that would be necessary to record it. In general, of course, Western European languages fail on both aims. You cannot tell how to pronounce an English word if you look at it, you cannot tell how to spell an English word unless you know it. But if one of the two aims is satisfactorily accomplished, it seems to me that as far as I know spelling systems, people nearly always will be willing to settle for that, and think of the result as a workable orthography.

KHALIL SEMAAN (Georgetown University): My question is directed to Dr. Politzer. You stated that in order to teach a language fruitfully we ought to teach the culture of the country whose language we are studying. I wonder if you can elaborate on this statement as to how to go about teaching this culture. 
ROBERT POLITZER: Well, I simply meant that in order to teach the meaning of the words, we have to analyze the situation in which the words are used and this inevitably leads us to the analysis of the foreign culture. Do you mean in a technical sense, just how you would teach the culture?

K. SEMAAN: Of course culture is always reflected in the language as being spoken, but you made the statement that to teach that language we ought to teach the culture of that country. Does that mean that we ought to give the students first a course in cultural anthropology which would deal with the culture as a whole of that country whose language they are studying?

POLITZER: No, I don't mean to imply anything like that. I think that in the normal teaching situation your cultural explanation or cultural analysis will be tied in with the teaching of the language. Quite clearly, if you teach a Spanish-speaking student about going to an American drug store, you have to explain to him what this particular cultural item is and the entire cultural complex that is connected with such a simple thing as an American drug store. So I think that your cultural explanation will develop out of your teaching situation rather than being presented by a parallel or preceding course in cultural anthropology.

K. SEMAAN: What does /a lali pala/mean?

WELMERS: Along with answering that question, I want to say that no one was bribed to ask it. /a lali pala/ means "he has made a hammock," and /a lâli palla/ means "he makes hammocks."

HAUGEN (Wisconsin): I would like to direct a couple of questions to Mr. Olmsted. I am a little troubled by the use of the word "frequency" and I wonder how deeply you thought through the concept of frequency. It seems to be used in different meanings in the different formulas. You have the frequency of phonemes which is one thing, that is to say, you have a reasonably finite number of phonemes, and the question of their frequency is a little different from the frequency of morphemes, for instance. Furthermore, when you speak of frequency of occurrence of a test word in the idiolect of a person, do you mean the number of times it occurs per ten thousand running words for instance, or some measure of that kind, and if so, just exactly how are you going to derive a sufficient body of text from the speech of individuals merely interacting in a social situation, and not writing down what they have said? It seems to me to be an absolutely insuperable 
task that you have set yourself when you consider how tentative and how inaccurate the measures of frequency are that we have even for English and the written materials in English. It seems to me that you are up against a very serious question and furthermore, it seems to me just on a personal basis of experience with language it is not the frequency per se but it is the usefulness of the word for which I don't see any particular measure available at the moment. A word may be very infrequent, let us say the parts of the spinning wheel, but anybody who wants to learn to spin has to learn those words. It is not a matter of their frequency, it's a matter of their absolute necessity in a particular situation. I think you have thought of that, it's perfectly obvious, but I just wondered why you didn't say anything about it. Furthermore, there are certain intangible qualities about words that are adopted. They are adopted because they are amusing, or attractive. There are qualities for which it would be very difficult to find any formula, words that we adopt simply because we think they are fun, or because they somehow create an atmosphere of congeniality between us and the members of whatever group we happen to belong to; those are some of the things about which I would like to have you talk a little more. As for the use of the word "Tory", I think you will recognize yourself how bad it is by the smirk with which you launched it-if I may use that term. The word "Tory" after all, in most cases represents one socially superior to the innovators, and therefore, it's going to be turned upside down in a good many of your formulations. The Tory who is of a lower status than the innovator adopts the word eventually because he has upward mobility. I also wonder about this term "upward mobility"-as to whether it is relevant in very many societies. It happens to be highly relevant in ours, but is it relevant in a European society with a highly structural class division, or in India?

DAvid L. OLMSTED (University of California, Davis): These questions you have raised are all very pertinent to the problem. Some of them I have thought about and consciously attempted to make provision for them here; others are new to me. For example, I hadn't thought of Tories ordinarily being socially superior. That didn't occur to me as a disadvantage of the term, but as I pointed out, I should not get involved in the term, and I am perfectly willing to drop it. But for societies where Tories are present I haven't stated that the 
Tories need necessarily have social status less than that of the model. All I stated is that if his social status is greater than that of the model, the chances are that he won't borrow a word from him. That probably has been held up in society as being the case, but let me attempt to deal with your questions in the o, der that you raised them:

Frequency, you are quite correct, is used in slightly differing meanings here. The calculations of frequency for both phonemic and morphemic purposes (purposes of calculating phonemic and morphemic regularity) are as I now see them, to be done on the basis of texts taken down from the language. I have considerable items of folk-lore from certain texts, I haven't gathered all the texts that I want to gather, but the main way in which such frequencies would be gathered would be the ordinary one of calculating the number of times a given phoneme occurs, and in this case, a given cluster. Now this is quite different, I grant, from the frequency count that I propose to take of occurrences of the test word in the model's speech while interacting with a Tory. This is something which you may well be right about when you say it is well nigh insuperable, but I am hoping that it isn't, because if it is insuperable, of course then I am in very grave difficulties indeed. What I propose to do is when a model is primed with the proper test word, to attempt to observe his interactions with the Tory in question, and simply to note down every time the test word occurs, ignoring the rest of the conversation. It may be better to test the frequency in a quasi-laboratory situation, small group situations to work out categories of interaction.

Now, another question you brought up is one that I had actually provided for in the system for some time and eventually cut it out, and that is, the relevance of the term. In working out these variables of course I simply attempted to think about studies of borrowing, and the sorts of terms that were borrowed in different situations of cultural contact, or situations in our own society, and I think it is very true that people do not borrow terms that are not relevant either to the model or to the Tory. That is, Tories don't customarily borrow terms from models which they do not feel are within the model's competence, and given the choice between two models, a person is more likely to borrow a term from that model that he feels controls his subject matter better than the other. Now I hope that I had taken care of that on the frequency of interaction and the frequency of occurrence point. That 
may not be the case, but it seems to me that if model and Tory interacted frequently and if the model used the given term frequently in this interaction with the Tory, then no matter how frequent the term may be in the language as a whole, I would predict innovation. I do not know, perhaps mere frequency of occurrence, frequency of interaction, will not do the trick. There are a number of other difficult points. One of them is the problem of rank, which was pointed out to me by Professor Murdock. It probably is a little too simple to assume that mere distance of rank differential will have an increasing effect on lexical innovations, for as he pointed out, studies of American society have shown that for a person whose rank is low in the hierarchy, differences between members whose ranks are high tend to be obscured. That is, a person whose rank is very low may not perceive those cues which differentiate the upper middle from the lower middle class, which are very clear to members of those two social classes in our own society. So that it may simply be that a sort of gross social status differential operates in that case, and that such fine distinctions are not known. 


\section{Special Paper}

\section{Current Trends in Language and Culture Programs in Western Europe}

\section{LOUIS LANDRE}

\section{Professeur d la Sorbonne. Professional Expert on the UNESCO Language Seminar, Ceylon, August, 1969}

I have seldom been so embarrassed as I am now in opening these brief remarks. I find myself in a group of highly specialized technicians and experts on language and linguistics whose learned papers and speeches I have listened to with considerable interest. My competence, if I have any, lies in a slightly different field. Many of my French and European colleagues would be far more qualified than I am to discuss linguistics and language teaching. Some of you will think that I should not have appeared here at all.

I agree with them to a considerable extent. In fact, after declining the honor of addressing this audience, I finally consented only on Professor Dostert's insistence, with the understanding that I should limit myself to general-some will say somewhat superficial-observations.

Insofar as I can observe, current trends in language and cultural programs in Western Europe, and principally in my country, are widely divergent from those which I have been able to observe during my tour of your universities, colleges and schools.

There are several reasons to that:

First of all, we have long discarded the grammatical approach in the study of modern languages and never feel that we still have to fight against it. It is more than fifty years ago, after the important work carried out in the fields of philology and linguistics in Germany, the Scandinavian countries, Great Britain, Switzerland and France principally, during the latter part of the 19th century, that the so-called "direct method," the oral-aural approach as you would say today, was generally accepted, with all that it implies, that is phonetic exercises, drills of all sorts, the initiation to structural patterns.

It was all the easier as our European systems of education have allowed us, for more than a half century, to start the study of lan- 
guages early, at the age of ten, and that, in the case of most public secondary schools, it can be continued for seven, eight or more years. This indeed obliged us to go slowly and progressively at the beginning, with only a timid use of phonetic symbols, but it has allowed us in the later stages in the secondary schools and at the university to go deeper into the study of the language, oral and written, in its present stage and in its original development, and to link it more closely for a much zreater number of pupils and students to the culture of the countries whose language they study.

Several consequences have followed both in our language studies and in our culture program.

The approach to linguistics has generally been and remains more influenced by historical, sociological, psychological and philosophical considerations in Europe than in other parts of the world. The influence for instance of Meillet, Vendryes, Henri Delacroix for instance is still strongly felt, as is exemplified by M. Marouzeau's various editions of La linguistique ou la science du langage, by the recent new edition in 1952 of Les Langues du Monde, a work of considerable importance, by the long list of publications of the Societé de Linguistique de Paris. European linguistics, as a rule, do not separate oral speech and the written language, often generally viewed in its historical perspective. Linguistic studies in the modern languages take advantage of what is done in the fields of the ancient languages.

It may very well be that in recent years descriptive phonetics and phonemics have been carried further in the U. S. A. than in Europe and I am sure our European colleagues in those fields are anxious to know what is going on here-however the work of Daniel Jones and Palmer is still carried on at the University College, London, and the Institute of Education in the University of London. The recent opening of an audio-visual center in France in connection with the Ecole Normale de Saint Cloud, the recent start of a journal on audio-visual aid, the activity of such scholars as Pierre Fouche, Gougenheim, Marguerite Durand are proof that in the country of Paul Passy, such studies are not neglected. Studies in historical phonemics have been carried on by Haudricourt and Julliand. Stress has recently been laid in the more practical fields on the length of vowels, the pitch, the grouping of sounds, the verbal intonation, the music of language and naturally on meaning, a certain danger having been felt to exist in analyzing and isolating too much. 
In the field of vocabulary, to mention only briefly the work of Benveniste, important studies have been made on the problem of frequency. If, as a rule, little favor has been shown on the Continent towards Basic English, efforts have been made principally in Belgium under Professor Closset, of the University of Liège, and in Scandinavian countries to stress the importance of a vocabulaire de base. Our colleague Michea in our Review Les Langues Modernes has published a most valuable series of articles on the methodology of word frequency, the importance of which will become evident with the Bibliography on the Teaching of Modern Languages now undertaken by UNESCO, in collaboration with your Modern Language Association and our Fédération Internationale des Professeurs de Langues Vivantes. For about three years now a special committee in France has been conducting a very precise investigation to establish plans for a vocabulaire élémentaire. All of the field work has been completed now by Professors Gougenheim and Aurélien Sauvageot and the work may come out by the end of this year or at the beginning of next.

A grammaire élémentaire will be the next step, not indeed as a means of bringing about a simplification of French grammar, which some had thought possible a few years ago, and for which indeed Paul Valéry had asked, but simply as a help to teaching. In the meantime, the studies in morphology and syntax, in philology, historical grammar and current grammar have been going on in the various countries of Europe. The publications of Professor Fernand Mossé, of the Collège de France, starting with his thorough and remarkable analyses of the periphrastic phrase "to be" plus the form in -ing in Old Germanic, Old English, Middle English, Modern English and continued with his studies on Gothic, Old and Middle English, those of Professor Zandvoort of Groningen (the Netherlands) on current English, the studies of some of our German colleagues on functional grammar are important contributions to be reckoned with.

If in the initial stages of language learning European teachers of languages have long discarded the translation method, if indeed they constantly insist on oral practice even at the university and graduate level, they feel that nothing solid has been accomplished until a subtle knowledge of the shades of meaning of words and idioms not only in oral speech but in delicate literary texts has been reached. This indeed we feel, is analysis of the most valuable kind and we carry it very far. The method of explication de textes which originated in France for 
the study of French texts more than a century ago has curiously enough spread to most other countries and served to further the close reading advocated by your new critics. We now use it extensively under various forms in secondary schools and our universities for the close study of foreign languages and literature. And, at the highest level, we advocate translation, translation from the foreign language into the mother tongue and also from the mother tongue into the foreign language not only as a test but as the only means of reaching the very spirit of foreign languages.

At this stage indeed as in the previous stages, language and culture cannot be and should not be separated, just as language and thought can never be dissociated, as was pointed out in 1924 by Dean Henri Delacroix in La. Langue et la Pensée and more recently in 1953 by Prof. Georges Gusdorf of the University of Strasbourg in La Parole.

The word culture itself is ambiguous, as was brought out two years ago in Brussels and last year at the International Conference at Sèvres when the problem of language study and culture was under discussion. To some, and this is largely the meaning of the German $K u l t u r$, culture means essentially the general social or sociological background: customs and institutions, ways of doing, connected with geographical conditions and eventually with an historical development. This we all feel in Europe and, I suppose, in the U. S. A., is essential in language teaching. I was glad to notice that the remarks on what you sometimes call "area" studies, what we in France call civilisation, met with general approval at the U.N.E.S.C.O. conference in Ceylon last year.

Beyond this, and we should be tempted to insist on this in France, language is the direct expression or a direct expression in a symbolic manner of thoughts and feelings, whether these are shared in common by a group of people, a nation for instance, or the people of several nations speaking the same language, or are simply those of an individual. Studies in the psychology of people have recently become more frequent and are almost always linked with the idioms of those people. For the other aspect of this question, we soon come to the problem of stylistics which is studied by many of us, or that of versification and poetic expression, a most delicate problem on the borderline between linguistics and literary criticism. It is noticeable that the latest volume, to my knowledge, published by the Sociêté de Linguistique de 
Paris, is precisely a study of this nature: No. LVI. Pierre Guiraud, Langage et Versification d'après l'œuvre de Paul Valéry. Etude sur la forme poétique dans ses rapports avec la langue. One of our recent theses in the English field, which incidentally I supervised, was that of $\mathbf{M}$. Léaud on poetic creation and versification in the poetical works of George Meredith.

We feel that at this level, and even at a much less advanced level there is a cultural value in the learning of foreign languages in that it is not only in all sorts of ways a training of the mind (provided the drill method is not overemphasized, which occasionally happens). It may throw a significant light on man, his very nature and, in some cases, his highest thoughts and aspirations. The remark was made very aptly yesterday morning that language which is a conventional structure cannot be dissociated from the human personality and "beyond the various and different structures, reflects man in his unity." Curiously enough in a series of coloquia we held in France previous to the U.N.E.S.C.O. conference, the humanistic aspect of language study was emphasized, and this point met with general approval both from the Eastern and Western members when it was made at the conference under the direction of Professor Anderson.

I am afraid I have greatly diverged from what is the original purpose of this conference. I shall be happy if I have helped you to understand that in our own ways in Europe we maintain a considerable interest in the study of languages. Perhaps more exchanges of informotion or contacts of this nature would not be altogether useless both to your country and my part of the world. 


\title{
VI. Closing Luncheon Speech
}

\section{A Humanist Looks at Linguistics}

\author{
HAYWARD KENISTON
}

Duke University

As I try to talk to you today, I have the strange feeling that if I have to talk about a humanist looking at linguistics, then I have to talk about myself looking at myself. At least for some time in my life I thought of myself as a linguist, because I had an interest in the study of language. However, at the time when I grew up-as a student and as a teacher-we were philologists. We did not know very much about linguistics in those days. Presumably the philologist was a man who loved rules; and I suppose that a linguist is a man who is interested in the operation of the tongue. There may or may not be a real difference between the two, but at any rate, I know that I grew up as one of those historical people who were concerned with how languages develop and change.

It is not surprising that the last half of the 19th century should have been primarily devoted to what I may call an evolutionary approach to the study of language. After all, those were the years when Darwin and his followers were talking about the historical evolution of the animal and physical world. And so the linguists-or the philologists-were also interested in the development of language, seeing a linguistic problem as one of change. And I must say, as I think back now, the reconstruction that they made of Proto-Indo-European, Proto-Romance-not to speak of Proto-Athabascan-was quite a formidable intellectual achievement. Somehow, on the basis of a study of historical documents, they were able to build a theory about a language of which we knew nothing else.

It is true that not infrequently they were rather uninterested in the sounds of language. They looked at the letters. I remember that when I first began to study Old Spanish we were taught that intervocalic $c t$ gave $c h$, as though by some subtle alchemy an $h$ was derived from a $t$, the $c$, however, remaining intact in this combination. And, of course, that weakness of historical and philological study persists, $\mathrm{n}$ a sense, to this day. The study of the development of sound resulted 
in a whole set of laws and rules, and somehow emerged in the most wonderful schematic patterns, so that everything seemed to happen according to what was then the current notion of physics. Physics operated by a set of laws, and so language too operated by a set of laws.

Towards the end of the century people began to shift their position. In Germany and in France, investigators began looking at actual language, the sounds of speech. That is when the lingua came in, and I suppose that is when we became linguists, because we were concerned with the physiological facts in the articulation of sounds. There is no need to tell you what a revolution that meant. It is not merely that people stopped looking at language as an historical evolution; they began to look at language as an instrument of communication, something immediate, present, or should I say synchronous, i.e. something that was to be described as happening-an event, a linguistic event. This was not limited to the field of phonetics. To show what it meant in regard to lexical units, or for any other aspect of a spoken and immediate language, I need only remind you of the old Linguistic Atlas of France.

Professor Landre this morning talked about the time fifty years ago when people in Europe began to look upon language no longer as a kind of hypothetical, historical conception but as actual human communication. It is puzzling to me that there should have arisen a feeling that linguistics is somehow in conflict with the study of the humanities. I think that the most striking trait of man is his gift for language. This is the trait that differentiates him; and if language is not a human function, what shall we talk about as being a human function? Over the years people have more and more, I think, tended to stress the human quality of speech. It do not want to put myself outside of linguistics just because I happen to be interested in the humanistic approach. As people all over the Western world began to re-examine the study of language as a system of communication, their methods of approach differed greatly. Some were interested in the merely mechanical aspect of language; many more have been interested in the cultural and socio-cultural aspect. And there have been others who were interested in the psychological or in the stylistic aspect. A great variety of interests in language have been conceived of, and all of them, I think, should be called linguistics unless we are going to be too narrow in our approach. 
I have a suspicion that you meant for me to talk about American linguistics, when you said, "A Humanist Looks At Linguistics." Certainly one does not look at American linguistics in the same way that one looks at Czechoslovak, or Swiss, or French linguistics, because it is quite different. I would like to say briefly what I think has been significant about American linguistics. It is quite typical of the American and pragmatic mind that we should want to look at the instrument of communication. We want to examine the sounds that are involved, the mechanical and purely physiological operations, the gradual development of a system of phonetics, and then a kind of language of phonetics, a shorthand of phonetics which we might call phonemics. The progress that we have made has been extraordinary, not only in the study of lesser known languages like the Indian languages or the unfamiliar languages of Africa, but also the Western languages. Studying the elements which make up the communication system, its sounds, its forms, and to a very great degree its syntax, we have learned a great deal about language. We have come away from the notion that language is normative, that it works according to a set of rules. We have limited ourselves to finding out the facts. But I must say that I have been somewhat troubled by the tendency of American linguists to look upon communication as a purely physical event. By this I mean the tendency to look upon communication merely as a series of wave lengths or other physical phenomena, as a series of neural impulses that are to be measured and calculated, but which are far removed from any meaning or value in terms of what one individual wants to convey to another individual. I remember a passage in Robert Hall's much discussed book Leave Your Language Alone! in which he says of syntax that there is not very much the linguist can do about it, because syntax deals with meaning. The passage was rather discouraging for me because, unfortunately, all my work in syntax had started with meaning and I wanted to know what other basis a language invents for expressing meaning, shades of meaning, subtleties of meaning. So I have been a little worried about the purely mechanical aspect of American linguistics; and yet, on the other hand, I would not want to discourage anybody from going ahead and carrying it out to its ultimate limit. Why should we not be able to take a discourse apart, analyze it and break it up into its segments? However, we should admit that we have to have non- 
segmental or supra-segmental or other elements in speech which are essential in communication.

This brings me to the other point that disturbs me about our contemporary approach to language in the United States, and that is that it seems to me to be too narrow in its conception of what the constituent elements of communication are. I do not think that quantity, intensity, and stress are suprasegmental at all. Of course, if we transcribe a communication on to a record, we have to put it in a linear demonstration, i.e. across a line on a page. But such a unilinear presentation of language is completely inadequate for a linguistic expression or experience. Language carries with it a whole set of other variations besides that of phonemes and morphemes, or even syntagmemes. Language is a series of events which we can break down, but which no listener ever does break down. What some of us have been teaching for years is that the unit of communication is not the phoneme or the phonetic unit, or even the syllable or the lexical unit, but the sentence. A sentence is a communication. No person receiving a communication goes through the process of analysis; he receives a single, highly complex, unitary communication, and he interprets it with all the $A B C$ 's and D's around it that are the result of his own particular experience in life.

Now, if these are weaknesses in contemporary American linguistics, and I believe them to be, what shall we hope about the future? I think nobody could have attended these meetings without being made aware of the infinite number of problems in the so-called "descriptive" technique that have not yet been solved. Everyone of us wants to encourage the continuous study of appropriate methods which will enable us to understand more fully the actual physical events that take place in a communication. But I do not think that this is all that we ought to be interested in.

If linguistics is a science, it obviously has to deal with objective criteria. But linguistics today is roughly in the stage which botany was in; back in the 18th century, which means it is still trying to describe elementary facts. The subsequent history of the natural sciences has gone in a different direction. Nobody would assert today that either botany or physics are descriptive sciences. The problems with which they are concerned now are highly theoretical and hypothetical. These sciences no longer rely on models and demonstra- 
tions that have visual reality, but operate in a world of pure fiction. I think we must do domething similar in the study of language.

Such a development, by the way, is not restricted to the natural sciences. From a primitive anthropology which tried to measure and calibrate the size of skulls and bones, we have moved into a highly speculative science, namely cultural anthropology. And the future of anthropology will not lie in further descriptions of human animals, but in the interpretation of genetics, or growth, or other problems relevant to human society and culture.

Even in psychology, another of the fledgling sciences, there has been a great change in the last 25 or 30 years. Psychologists started out by studying how rats behaved under certain stimuli and from there they went on to interpret human behavior as caused by certain stimuli. But they did not make any connection with what was going on in man's mind: they only observed behavior. I do not have to remind you that psychology has gone in wholly new directions today. The most fruitful of these is the field of social psychology and its interest in the behavior of large groups as well as that of individuals. It is very interesting that psychology is following the same path as physics, inasmuch as it is trying to determine fundamental problems and the hypotheses on which to establish psychological theory.

In American linguistics, it seems to me, on the whole, we have been rather afraid to venture over into the field of speculation. For Europe this is not true at all. The Europeans have always been speculative, but we in this country have not wanted to speculate. As scientists, I think, we have good grounds for entering the field of theoretical or philosophical speculation in order to establish a set of assumptions about language which we can then analyze and attempt to interpret as to whether or not they are valid. We should ask ourselves what is the larger significance of language as an instrument for conveying thought and feeling.

There are a few points which came up in the meetings yesterday and today on which I would like to make some comments. Somebody said that when people can not find the answers in modern linguistics, they talk about free variation. That reliance on free variation has always irritated me. At least as far as syntax is concerned, I am convinced that there is no such thing as free variation. Two expressions, even though they look alike at first glance, do not convey the same emotion. 
Every way of saying something has its unique and particular communication to some other individual. Otherwise, we would be reducing our analysis to the level of mere denotative statements or commands, but this is only a very small portion of communication. Communication is made up of all the things that are in our mind, of all our dreams, our hopes, and our desire to control other people, to influence them. In other words, the art of rhetoric is an important part of any communication that we make. And so, every phase of a linguistic communication is relevant to its ultimate purpose, namely the meaning which it conveys. The aim of our study must be to arrive at as clear a picture as possible of how language conveys the attitudes as well as the ideas of an individual to another individual or to a group.

Here is another point on which I want to comment. As Mr. Landré said this morning, the study of linguistics is not limited to merely oral communication. Language, after all, has received a kind of form, arbitrary if you will, but nonetheless significant, in the written word. In the written word we find the use of the basic instrument of speech translated into a more formal or more standardized expression, capable of conveying the same kind of ideas, but no longer simply as statements and questions, but clad in forms of beauty, of emotion, of revelation. If language were only a device for giving orders or imposing our will upon other people, then it would be a rather poor and unsophisticated instrument. Language is much more than that. It is the greatest treasure that man has, because it is a means for explaining and interpreting his ideal of life and his experience in life. I should hope that linguistics in this country would more and more be concerned with the total body of linguistic communication. The kind of studies made by Leo Spitzer and by Karl Vossler before him, are not measurable, perhaps, but they are nevertheless important for us. They are speculative and highly individual, it is true. But are we going to give up the speculative and personal interpretation of language in the name of an objectivity which all people will accept? I resent this, because I do not want conformity.

Let me say a few words about the future of American linguistics. I think all of us who are on the fringe and not in the center of things, have been somewhat perturbed at a kind of arrogance which is observable among modern linguists. I do not know why there is this strange polemicism that goes on in the field of linguistics. This does 
not refer to American linguists alone, nor is it particularly modern. It was characteristic of philology in the 19th century too, with the Homeric laughter still resounding in Europe over this or that blunder made by some philologist.

Let us be humble in our particular contribution and let every one who is dedicated to a particular research job pursue his way. But let him not think that his is the only way. If we are humble about our own contribution, we must have a certain tolerance towards the man who is doing something quite different. Although other workers in our field may be doing things that are different from ours in method and goal, they all have in mind the ultimate purpose of the common cause, which is to know more about how language functions. If the linguist remembers this, he will move closer to other fields, as, after all, we must. We must establish a closer relationship between linguistics and the whole field of anthropology, and I would suppose also to psychology and the other social sciences. At least in part, through the psychologist we will have to approach one problem which is very important, but about which very little has been done up to the present time. It is the problem of understanding language as the unique expression of an individual personality. In a very large measure we will also have to approach this problem through the study of literature. Within our sphere of interest lies not only the social function of language, but also the individual function of language. It is not enough merely to count the number of phonemes or symbols. That is only the first step. The ultimate step is to understand language as a function of the human mind.

Language as a unique human instrument should not be separated from the humanities of which it is inevitably a part. It is a part which has its own and rather specialized techniques, and yet these techniques must lead to the common goal, which is the study of man. The study of man can not be based on a merely physical examination of either his shape and size or of the forms and ways in which he speaks. It can only depend upon what happens to him as a human spirit. 


\section{APPENDIX 1}

\section{Program of the Fifth Round Table Meeting}

FIRST SESSION-Friday, April 30, 1954, 9:30 A.M.

Welcoming Remarks:

Reverend Frank L. FadNer, S. J. (Executive Assistant to the Regent, School of Foreign Service, Georgetown University)

Archibald A. HiLl (Vice-Director of the Institute of Languages and Linguistics)

Panel: Bilingualism and Mixed Languages

Chairman: Einar Haugen (University of Wisconsin) Problems of Bilingual Description

Speakers:

WERNER F. LEOPOLD (Northwestern University) $A$ Child's Learning of Two Languages

ERnest HAden (University of Texas) The Phonemes of Acadian French

URIEL WeINREICH (Columbia University) Linguistic Convergence in Immigrant America

LUNCHEON MEETING-Friday, April 30, 1:15 P.M.

Speaker: Norman A. McQuown (University of Chicago) Cultural Implications of Linguistic Science

SECOND SESSION-Friday, April 30, 1954, 2:30 P.M.

Panel: The Spectrographic Analysis of Speech

Chairman: Gordon E. Peterson (University of Michigan) Acoustical Vowel Relationship

Speakers:

MorRIs Halle (Massachusetts Institute of Technology) Why and How Do We Study the Sounds of Speech?

Ted D. HANLEY (Purdue University) Time Measurements in Speech Analysis 
RECEPTION-Friday, April 30, 1954, 5:30 P.M.

Offered by Georgetown University, Gymnasium Band Room, on the Campus

THIRD SESSION—Saturday, May 1, 1954, 10:00 A.M.

Panel: Language and Culture

Chairman: Mrs. Regina Flannery-Herzfeld (Catholic University)

Speakers:

Robert PolTtzer (Harvard University) Developing Cultural Understanding through Foreign Language Study

David Olmsted (University of California, Davis) Towards a Cultural Theory of Lexical Innovation

PaUl Garvin (Georgetown University) Literacy as a Problem in Language and Culture

William E. Welmers (Cornell University) Non-segmental Elements in Foreign Language Learning

Special Paper: Current Trends in Language and Culture Programs in Western Europe. Louis Landré (Professeur à la Sorbonne. Professional Expert on the UNESCO Language Seminar, Ceylon, August, 1953)

CLOSING LUNCHEON-Saturday, May 1, 1:00 P.M.

Offered by Georgetoun University

Speaker: HaYwaRd Keniston (Duke University) A Humanist Looks at Linguistics 


\title{
APPENDIX 2
}

\section{Membership of the Fifth Round Table Meeting on Linguistics and Language Teaching-30 April-1 May, 1954}

\author{
Allen, Rolfe, L., United States Army \\ Alter John V., Howard University \\ Andersson, Theodore, Yale University \\ Ani, Moukhtar, Georgetown University \\ Antonoff, Anna N., Georgetown University \\ Baltazar, Jesse M., Georgetown University
}

Bargin, Germaine, U. S. Department of Agriculture, Washington, D. C.

Barritt, Westbrook, Washington \& Lee University, Lexington, Virginia

Bellerose, Leo M., Georgetown University

Bjelajac, Slavko N., Georgetown University

Bodman, Nicholas C., Foreign Service Institute

Bonianovsky, Eugenie, Georgetown University

Bowen, Jean Donald, Foreign Service Institute

Bretan-Le Bovit, Judith

Brooks, Nelson, Yale University

Butler, Joseph, Jr., Howard University

Cabaud, Jacques M., Howard University

Caino, Domingo, Georgetown University

Casagrande, Joseph B., Social Science Research Council

Castiglione, Salvatore J., Georgetown University

Chén, Julia I. H., United States Navy

Chesnut, George L., Jr., Arlington, Va.

Choseed, Bernard, Georgetown University

Clark, William H., Jr., University of Rochester, New York

Coutinho, Joaquín, de S., Georgetown University

Cowan, J. M., Cornell University

Cutling, Helen F., W. C. U. N. C., Greensboro, N. C.

De Francis, John, Georgetown University

deMandach, A. B., Central Michigan College

Dwaink, John, Union College, Schenectady, N. Y.

Eddy, Frederick D., Hood College, Frederick, Md.

Fadner, Rev. Frank L, , Georgetown University

Fallis, Roy F., Jr., Georgetown University

Fersen, Nicholas, Georgetown University

Flannery-Herzfeld, Regina, Catholic University

Francis, Sister, S. N. D., Trinity College

Frith, James R., USAF Institute of Technology

Gardiner, Catharine, Wilson Teachers College

Garey, Howard, Yale University 
Garvin, Paul L., Georgetown University

Gibbs, John W., Sanz School, Washington, D. C.

Gorosch, Max, Stockholm University, Stockholm (Sweden)

Grigaut, H. L., Georgetown University

Guevard, John A., Lt. Col., Fort Holabird, Maryland

Haden, Ernest F., The University of Texas

Haines, S. M., Major, United States Army

Halle, Morris, Massachusetts Institute of Technology

Hanhardt, Arthur M., University of Rochester, New York

Hanley, T. D., Purdue University

Haugen, Einar, University of Wisconsin

Hill, A. A., Georgetown University

Hirsch, Ruth, Georgetown University

Hodge, Carleton T., Foreign Service Institute

Horn, Stefan F., Georgetown University

Hughes, George W., Massachusetts Institute of Technology

Hundley, Mary G., Washington, D. C.

Jenks, Audrie, St. Agnes School, Alexandria, Va.

Kalikin, E., Georgetown University

Keniston, Hayward, Duke University

Kepke, John, American Council of Learned Societies

Kiernan-Vasa, Helen, Studio of English, Washington D. C.

Krader, Mr. \& Mrs. Lawrence, Washington, D. C.

Krassovsky, Collerohé, Washington, D. C.

Landré, Louis, Sorbonne, Paris

Lang, Anton, Georgetown University

Le Bovit, Harry

Lee, Eunice Day, Howard University

Leopold, W. F., Northwestern University

Levitsky, Sergei A., Georgetown University

Loughran, Clayton D., Georgetown University

Lucyanow, Ariadne, Georgetown University

McQuown, Norman A., University of Chicago

Magner, Thomas F., University of Minnesota

Mahaliev, Erdni, Georgetown University

Margaret Thérèse, Sister, S. N. D., Trinity College

Martínez, M. G., Georgetown University

Mayer, Edgar W., Lafayette College, Easton, Pa.

Melignani, Antoinette, L. A. B., Trinity College

Moore, Omar K., Naval Research Laboratory, Washington, D. C.

Moore, Paul, Northwestern University

Morgan, Raleigh, Jr., North Carolina College, Durham,

Mueller, Hugo, Georgetown University

Myshencoff, Nicholas, Georgetown University

Neledinsky, Eugene, Georgetown University

Norton, Virginia, Georgetown University 
Obrecht, Dean H., Lafayette College, Easton, $\mathrm{Pa}$.

Obolensky, S., Georgetown University

Olmsted, D. L., University of California, Davis

Olshansky, Boris, Georgetown University

Ornstein, Jacob, United States Department of Agriculture, Washington, D. C.

Pavia, Mario N., Georgetown University

Peterson, Gordon E., University of Michigan

Pickett, J. M., United States Air Force

Politzer, R. L., Harvard University

Poushkin, A. M., Georgetown University

Ramras, Herman, University of Minnesota

Rathjens, Clara, Trinity College

Rice, Frank A., Foreign Service Institute

Sa'id, Majed, Georgetown University

Sanz, Robert B., Sanz School, Washington, D. C.

Schulgin, Dimitry, Georgetown University

Scoltron, Edith, Hiram College, Ohio

Seiler, Hansjakob, Rockefeller Foundation, New York

Semaan, Khalil, Georgetown University

Shnitnikoff, Boris N., Georgetown University

Smitb, Philip H., Jr., University of Pennsylvania

Stanley, Mildred C., Washington, D. C.

Stevens, Mrs. Lisbeth, Johns Hopkins University

Stockwell, Robert, Foreign Service Institute

Sushko, Michael, Georgetown University

Trager, Edith, Wasbington, D. C.

Trager, George L., Georgetown University

Tuniks, S., Georgetown University

Viglie, F., Yale University

Vinay, Jean Paul, University of Montreal

Virski, Olga Scherer, Yale University

Waller, Claire, Baltimore Friends School

Weinreich, Uriel, Columbia University

Welmers, William E., Cornell University

Wilhelm, Maria, Howard University

Wyatt, Kathryn Day, American University

Young, Elizabeth Jen, Georgetown University

Young, John, Georgetown University

Zarechnak, M., Georgetown University

Zelensky, Paul, Georgetown University 



\section{APPENDIX 3}

\section{Index of Speakers}

Fadner, Father Frank L., S. J...................... 1

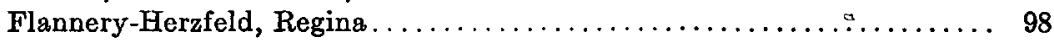

Garvin, Paul L $154 \ldots \ldots \ldots \ldots$

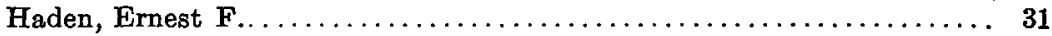

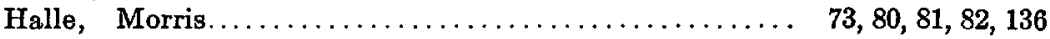

Hanley, T. D........................ 83, 95, 96, 97

Haugen, Einar. . . . . . $\ldots \ldots \ldots \ldots \ldots \ldots \ldots \ldots, 50,51,53,80,95,96.137$

Hill, A. A............................... 136

Hodge, Carleton. . . . . . . . . . .

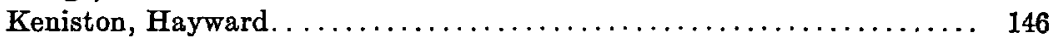

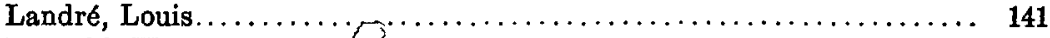

Leopold, Werner F.............................. 51

Martínez, M. G............................... 53

MeQuown, Norman A. . . . . . . . . . . . . .

Mueller, Hugo. . . . . . . . . . . . . . . . . . . . . . . . . . 50

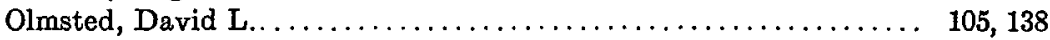

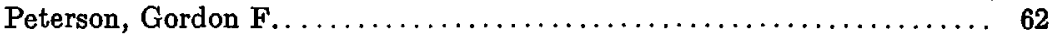

Politzer, Robert $\mathbf{L} \ldots \ldots \ldots \ldots \ldots \ldots \ldots \ldots \ldots \ldots \ldots \ldots \ldots \ldots \ldots \ldots ., 99,137$

Ramras, Herman............................... 5

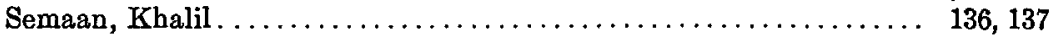

Stockwell, Robert.......................... 82, 83, 95, 96

Vinay, Jean Paul............................... 49, 96

Weinreich, Uriel . ............................. 40

Welmers, William E......................... 52, 130, 137 